\title{
Lectures on Random Schrödinger Operators
}

\author{
Peter D. Hislop
}

This paper is dedicated to Jean-Michel Combes on the occasion of his sixty-fifth birthday.

\begin{abstract}
These notes are based on lectures given in the IV Escuela de Verano en Análisis y Fisica Matemática at the Instituto de Matemáticas, Cuernavaca, Mexico, in May 2005. It is a complete description of Anderson localization for random Schrödinger operators on $L^{2}\left(\mathbb{R}^{d}\right)$ and basic properties of the integrated density of states for these operators. A discussion of localization for randomly perturbed Landau Hamiltonians and their relevance to the integer quantum Hall effect completes the lectures.
\end{abstract}

\section{Contents}

1. Models of Random Media 1

2. Basic Results in the Theory of Random Operators 14

3. One-Parameter Families of Operators: Spectral Averaging and Perturbation of Singular Spectra

4. The Wegner Estimate and the Integrated Density of States 36

5. Resolvent Estimates and Multiscale Analysis 48

6. Localization for Families of Random Schrödinger Operators

7. Random Magnetic Schrödinger Operators and the Integer Quantum Hall Effect

8. Fixed-Energy Multiscale Analysis $\quad 78$

References

1991 Mathematics Subject Classification. Primary 81Q10, 35P05; Secondary 47A55.

Key words and phrases. Random operators, Localization, Wegner estimate, Density of states. The author was supported in part by NSF Grant \#0503784. 


\section{Models of Random Media}

1.1. Introduction. In this chapter, we present an overview of the spectral theory of random media discussed in these notes. We are primarily concerned with the phenomena of localization for electrons and for classical waves propagating in randomly perturbed media. By localization, we mean that the permissible states of the system, with energies in a specified range, do not propagate in time and are localized in space. These states correspond to the existence of point spectrum for the corresponding self-adjoint operators describing the system. The fundamental idea is that the random perturbations cause states, which would otherwise be extended throughout all space, to effectively localize in a finite region due to multiple backscattering off of the random potential. Spectrally, these models are interesting because the spectrum in an interval does not consist of discrete eigenvalues but rather the eigenvalues are dense in the spectrum in the interval with probability one. Dynamically, states with energies localized in such intervals are characterized by the fact that the corresponding moments of the time-dependent position operator are bounded for all time. This effect is referred to as dynamical localization.

1.2. Physical Considerations from Condensed Matter Physics. The one-electron model of condensed matter physics is a simplification which has had some remarkable success in describing certain properties of crystals. The basic notions of insulating, conducting, and semiconducting materials can be explained by the energy spectrum of a single electron moving under the influence of a periodic array of atoms, and the Pauli exclusion principle. The latter determines the occupation of states by noninteracting electrons. In the one-electron model, the interaction between the electrons is neglected and the spectral and transport properties of the material is described by a one-particle Schrödinger operator $H_{0}$. We emphasize that another approximation implicit in the one-electron model is that the media is infinitely-extended in space. Under this assumption, we can neglect any boundary effects and use techniques common in statistical mechanics. In particular, we will often approximate the infinitely-extended system by a finitely-extended one. We will study the dependence of relevant properties of the system on the volume of the region, and then take the volume to infinity to recover the properties of the extended system. We refer to this as the thermodynamic limit.

The actual one-electron model is determined by the choice of the potential $V_{0}$, and the presence of external fields. For example, a perfect crystal is described by a periodic potential function $V_{0}$. The one-electron model is given by the Schrödinger operator $H_{0} \equiv K+V_{0}$, where $K$ describes the kinetic energy of the particle. For example, the choice $K \equiv-\Delta$ describes the kinetic energy of a free particle. We can make other choices for the operator $K$. A particle moving in the presence of an external electric field is described by $K \equiv-\Delta+F \cdot x$, where $F$ is a constant vector representing the electric field. A particle in a magnetic field is described by the Schrödinger operator $K \equiv(-i \nabla-A)^{2}$, for a vector potential $A$, so that the magnetic field is $B=d A$ (that is, $B=\nabla \times A$ in two- and three-dimensions).

We will always assume that the spectral properties of the background Schrödinger operator $H_{0}=K+V_{0}$, for operators $K$ given above, are, in some sense, completely known. We are interested in studying perturbations of $H_{0}$. As we consider systems that are infinitely-extended in space, we will be primarily concerned with perturbations which do not decay at infinity. Such perturbations have the possibility of 
radically changing the spectrum of $H_{0}$, as we shall see. There are other, interesting phenomena associated with compactly-supported perturbations of $H_{0}$, such as the occurrence of impurity levels in band gaps (see, for example $[\mathbf{3 7}, \mathbf{4 9}]$ ). Our most important example is the description of impurities in an infinitely-extended crystal. The impurities are described by a perturbation $V_{1}$. Thus, one is lead to the study of Schrödinger operators of the form $H=K+V_{0}+V_{1}=H_{0}+V_{1}$.

Let us consider the simple case $H_{0}=-\Delta+V_{0}$ further. It is well-known [113] that in the absence of any perturbation, a Schrödinger operator $H_{0}$, with $K=-\Delta$ and a continuous, periodic potential $V_{0}$, has purely absolutely continuous spectrum. As a set, the spectrum of $H_{0}$, which we denote by $\sigma\left(H_{0}\right)$, is a union of closed intervals $B_{n}$, called bands. An energy $E \in B_{n}$ represents an energy at which an electron can propagate through the crystal. If two bands are separated, the intervening open interval is called a spectral gap. The occurrence of a gap in the spectrum, which represents forbidden energies at which an electron cannot propagate, provides one of the bases of our understanding of the electronic properties of crystals.

We point out that our terminology of energy bands and gaps includes the following cases. Many Schrödinger operators of the form $H_{0}=-\Delta+V_{0}$ have spectrum filling the half-line $\left[M_{0}, \infty\right)$, for some constant $M_{0} \in \mathbb{R}$. This is the case, for example, if $V_{0}$ is positive, bounded, and has compact support. In this case, we say that $H_{0}$ has one band $\left[M_{0}, \infty\right)$ and one gap $G \equiv\left(-\infty, M_{0}\right)$. The free Stark Hamiltonian $H_{0}=-\Delta+F \cdot x$ has spectrum equal to $\mathbb{R}$. This operator has one band $B_{1}=\mathbb{R}$ and no gap. As another extreme case, the Landau Hamiltonian $H_{A}=(-i \nabla-A)^{2}$, in two-dimensions, with vector potential $A\left(x_{1}, x_{2}\right)=(B / 2)\left(-x_{2}, x_{1}\right)$, has only pure point spectrum with infinitely-degenerate eigenvalues given by $E_{n}(B)=(2 n+1) B$. We call the infinitely-degenerate energy levels $E_{n}(B)$ the bands and the open intervals $G_{0} \equiv\left(-\infty, E_{1}(B)\right)$, and $G_{n} \equiv\left(E_{n}(B), E_{n+1}(B)\right)$, for $n \geq 1$, the gaps.

Returning to our description of the one-electron model for a perfect crystal, let us consider why we are interested in perturbations of $H_{0}$ that do not decay at infinity. The goal in the study of electrons in crystals is a description of finite conductivity. Classically, conductivity $\sigma$ is the constant of proportionality between an applied electric field $E$ and the induced current $J$ so that we have Ohm's law $J=\sigma E$. The current $J$ corresponding to an electron state $\psi$ in a one-electron model is the electric charge $e$ times the matrix element of the velocity operator $v=\frac{d X(t)}{d t}$, so we have $J=e\langle\psi, v \psi\rangle$. The operator $X(t)$ is the Heisenberg position operator (see (1.2) below). In a perfect crystal, the static conductivity is defined to be $\sigma \equiv$ $\lim _{|E| \rightarrow 0}|J(E)| /|E|$. It is known that the static conductivity for a perfect crystal is infinite [74]. Anderson [6] proposed that impurities and defects might explain the observed finiteness of electric conductivity of crystals at zero temperatures (at finite temperatures electron-electron and electron-phonon collisions become important mechanisms in resistivity). In the one-electron picture, impurities are described by a nonperiodic perturbation $V_{1}$ of the periodic atomic potential. Thus, one is lead to the study of Schrödinger operators of the form $H=-\Delta+V_{0}+V_{1}$, where $V_{0}$ is periodic and $V_{1}$ represents the impurities.

How shall we choose the perturbation $V_{1}$ ? It follows from the basic stability of the absolutely continuous spectrum [75] that the impurity potential $V_{1}$ must be extended in space and nondecaying if it is to change the qualitative nature of the absolutely continuous spectrum or the spectral type. If the impurity potential $V_{1}$ has compact support or is rapidly decaying as $|x| \rightarrow \infty$, then the absolutely 
continuous spectrum of $H_{0}$ is unchanged [112], and at most some eigenvalues are added in the spectral gaps (as mentioned above, this is an interesting problem in itself). For example, if the potential $V_{1}$ is such that the pair of Hamiltonians $\left(H_{0}, H\right)$ has a conjugate operator $A$ in the sense of Mourre theory, then $H$ is also ballistic [24] meaning that $X^{2}(t) \sim t^{2}$, and the electron motion resembles free motion. Clearly, such motion corresponds to infinite conductivity which is what we are trying to eliminate through the perturbation $V_{1}$. It follows that our impurity potential $V_{1}$ cannot be concentrated in space or decaying at infinity, but must be present throughout the infinite crystal. The investigation of such perturbations is difficult and requires new tools.

In these notes, we will concentrate on the study of the spectral properties of Schrödinger operators $H_{V_{1}}=H_{0}+V_{1}$, where $H_{0}=K+V_{0}$ is assumed to have known spectral properties, and the perturbation $V_{1}$ belongs to a class of infinitely-extended perturbations. We will consider the following questions.

\section{Spectral Properties of $H_{V_{1}}$.}

(1) What is the spectrum of $H_{V_{1}}$ as a set?

(2) What is the spectral type of $H_{V_{1}}$ ?

(3) What is the nature of the eigenfunctions, if there are any?

The study of transport properties of the one-electron model involves the determination of the long-time asymptotic behavior of the matrix elements of the moments of the position operator $X(t)$. Basic questions which are of interest here include

\section{Transport Properties of $H_{V_{1}}$.}

(1) Is the motion generated by $H_{V_{1}}$ ballistic, diffusive, or localized?

(2) What is the conductivity of the material characterized by $H_{V_{1}}$ ?

(3) How stable are the transport exponents under perturbations?

As can be imagined, we need to restrict the class of allowable perturbations in order to address these questions. It turns out that if $V_{1}$ belongs to a family of perturbations which form a stochastic process $V_{\omega}$, and we consider the family of operators $H_{\omega}=H_{0}+V_{\omega}$, then we can provide detailed answers to the above questions. The introduction of a family of perturbations, rather than just one perturbation, allows us to make a general spectral analysis which holds for almost every perturbation in the family. The ability to average over potentials in the family provides us with a powerful tool for spectral analysis. The general framework for our investigations, then, will be a family of self-adjoint operators $H_{\omega}$, where $\omega \in \Omega$ describes a possible realization of the random media. These realizations occur with a probability given by a measure $\mathbb{P}$ on the space of all possible configurations $\Omega$. We will make this picture more precise in chapter 2 .

1.3. Geometric Considerations from Deterministic Models. As an introduction to the manner in which an infinitely-extended perturbation (that is, a potential $V$ which does not vanish at infinity) influences the spectral properties of the Laplacian, we review some results of a geometric character. Many spectral and transport properties of a Schrödinger operator $H_{V}=-\Delta+V$ depend on geometric characteristics of the potential $V$. There now exist 1) geometric conditions on $V$ which imply the absence of absolutely continuous spectrum, 2) geometric conditions on $V$ which imply the absence of diffusion, and 3) lower bounds on the moments of the position operator in terms of the fractal dimension of the spectrum for a general self-adjoint operator $A$. 
The absence of absolutely continuous spectrum and the absence of diffusion require geometric conditions on the potential $V$ which insure that the particle does not freely propagate to infinity. In particular, its motion does not resemble that of a free particle. Such potentials are characterized by regions where they are large in the sense of the Agmon metric for a given interval of energy. The general results indicate that these effective barrier regions can be sparsely distributed provided they dominate the potential in the complementary region. The conditions on the potential depend on the dimension. Examples of such geometric conditions can be found in the papers $[\mathbf{1 0 3}, \mathbf{1 2 4}]$.

This type of result on the absence of absolutely continuous spectra, and the absence of diffusion discussed next, require careful estimates on the boundary-value of the resolvent $R(E+i \epsilon)$ as $\epsilon \rightarrow 0$. Absence of absolutely continuous spectrum follows from the estimate

$$
\sup _{\epsilon \rightarrow 0}\|R(E+i \epsilon) \phi\|<C_{0},
$$

for a finite constant $C_{0}$ and any function of compact support $\phi$. The main techniques used to establish this type of estimate include the geometric resolvent formula, the spectral stability results of Briet, Combes, and Duclos [18], Agmon exponential decay results [1], and deBrange's theorem on the boundary-values of operatorvalued Herglotz functions.

There are far fewer results on sufficient conditions for the absence of diffusion for Schrödinger operators. Montcho [104] has discovered particularly nice, dimensionindependent conditions on the potential $V$ which guarantee the absence of diffusion. For a Schrödinger operator $H$, the dynamics is generated by the unitary group $U(t)=e^{-i H t}$. For a state $\psi$, the mean-square displacement is defined by

$$
\left\langle\psi, U(t)^{*} X^{2} U(t) \psi\right\rangle \equiv\left\langle X^{2}(t)\right\rangle_{\psi} .
$$

Roughly speaking, the dynamics is called diffusive if there exists a nonzero constant $D$ so that

$$
\left\langle X^{2}(t)\right\rangle_{\psi} \sim D t
$$

The absence of diffusion means that $D=0$, that is, the left side of (3) grows more slowly than $t$. Let the time-averaged mean-square displacement be denoted by

$$
\left\langle\left\langle X^{2}\right\rangle\right\rangle_{T} \equiv T^{-1} \int_{0}^{T}\left\langle X^{2}(t)\right\rangle_{\psi} d t
$$

There are sufficient geometric conditions on the potential $V$ so that

$$
\limsup _{T \rightarrow \infty} T^{-1}\left\langle\left\langle X^{2}\right\rangle\right\rangle_{T}=0
$$

which implies the absence of diffusion.

Another topic concerns the relation between the fractal properties of the spectrum of a self-adjoint operator $A$ and the long-time asymptotic behavior of the time evolution $U(t)=e^{-i t A}$ generated by $A$. Given any state $\psi$, a spectral measure $\mu_{A, \psi}$ for $A$ and $\psi$ is defined by the spectral theory through the representation

$$
\langle\psi, U(t) \psi\rangle=\int_{R} e^{-i t \lambda} d \mu_{A, \psi}(\lambda),
$$

Barbaroux, Combes, and Montcho [10], and Last [98] proved that the Hausdorff dimension of the measure $\mu_{A, \psi}$ determines a lower bound on the $\alpha$ moment of the 
position operator $X$ defined as in (4) with $\alpha$ replacing 2 . They prove that for sufficiently regular states $\psi$ and for any $\epsilon>0$, there is a constant $C_{\psi, \epsilon}$, so that

$$
\left\langle\left\langle X^{\alpha}\right\rangle\right\rangle_{T} \geq C_{\psi, \epsilon} T^{\frac{\alpha}{d}\left(A_{\psi}-\epsilon\right)},
$$

where $A_{\psi}$ is the Hausdorff dimension of the spectral measure $\mu_{A, \psi}$. This work requires a firm understanding of spectral theory and the classical analysis of measures. Many recent works are devoted to estimating the diffusion exponent from below, and we mention $[\mathbf{2 3}, \mathbf{1 2}]$.

1.4. Anderson Tight-Binding Model. The simplest model that describes the propagation of a single electron in a randomly perturbed crystal is called the Anderson tight-binding model. Atoms are considered to act on the electron at a single site only and short distances are eliminated by restricting the electron motion to the points of the lattice $\mathbb{Z}^{d}$. This eliminates short distances and provides a highenergy cut-off for the free kinetic energy.

We consider an operator of the form

$$
H=\Delta+\lambda V_{\omega}^{A}, \text { on } l^{2}\left(\mathbb{Z}^{d}\right) \text { for } d \geq 1 .
$$

The finite-difference Laplacian $\Delta$ is defined by

$$
(\Delta f)(n)=\sum_{|n-m|=1} f(m) .
$$

This operator describes the kinetic energy of an electron moving on the lattice. By the Fourier transform, one finds that the spectrum is purely absolutely continuous and equal to

$$
\sigma(\Delta)=[-2 d, 2 d] .
$$

The Anderson-type potential $V_{\omega}^{A}$ is constructed as follows. Let the family

$$
\left\{\omega_{i}\right\}, i \in \mathbb{Z}^{d},
$$

be a stochastic process indexed by $\mathbb{Z}^{d}$. Each random variable is distributed identically with a distribution function

$$
g(\omega) \geq 0,
$$

which we assume has bounded support. We consider the random variables to be independent. For simplicity, we take

$$
\operatorname{supp} g=[-1,1]
$$

The potential $V_{\omega}^{A}$ is defined by

$$
\left(V_{\omega}^{A} f\right)(m)=\omega_{m} f(m), \text { for } m \in \mathbb{Z}^{d} .
$$

It is often convenient to add a coupling constant $\lambda$ in front of the potential which measures the strength of the interaction and as such is a measure of the disorder of the system. More generally, the disorder of a random system is usually taken to be $\|g\|^{-1}$, where $g$ is the normalized density. The range of the scaled potential $\lambda V_{\omega}^{A}$ is $[-\lambda, \lambda]$. The totality of the independent random variables allows us to construct a probability space $[-1,1]^{\mathbb{Z}^{d}}$, with the measure $P$ induced by the product measure.

The Anderson tight-binding model is

$$
H_{\omega}(\lambda)=\Delta+\lambda V_{\omega}^{A} .
$$


This Hamiltonian $\mathbb{Z}^{d}$-ergodic with respect to the translation group on the lattice. By Pastur's theorem, the family of operators has a deterministic spectrum $\Sigma$. This means that there exists a fixed subset $\Sigma \subset \mathbb{R}$ such that

$$
\text { IP - a.s. } \sigma\left(H_{\omega}\right)=\Sigma \text {. }
$$

Theorem 1.1. For $\mathbb{P}$-almost every $\omega \in \Omega$, the spectrum $\sigma\left(H_{\omega}\right)=\Sigma$, and $\Sigma=[-2 d, 2 d]+\lambda \operatorname{supp} g=[-2 d-\lambda, 2 d+\lambda]$.

Our object of study is the spectral type of the random family $H_{\omega}$ for almost every $\omega \in \Omega$. We can already make some preliminary observations with respect to the spectral type.

(i) The spectrum $\sigma(\Delta)=[-2 d, 2 d]$ is absolutely continuous since the operator $\Delta$ is unitarily equivalent to multiplication by $2 \sum_{j=1}^{d} \cos \left(2 \pi k_{j}\right), k_{j} \in[0,1]$, by the Fourier transform.

(ii) $\sigma\left(V_{\omega}\right)$ is pure point. The vector $\delta_{n}(m) \equiv\left\{\begin{array}{ll}1 & n=m, \\ 0 & \text { other, }\end{array}\right.$ satisfies

$$
\left(V\left(\delta_{n}\right)\right)(k)= \begin{cases}\omega_{n} & n=k, \\ 0 & \text { other, }\end{cases}
$$

that is

$$
V_{\omega} \delta_{n}=\omega_{n} \delta_{n}
$$

so each vector $\delta_{n}$ is an eigenfunction for $V_{\omega}$. Hence, the almost sure spectrum of $V_{\omega}$ is $[-1,1]$ and dense pure point.

Although this model seems extremely simple, there are some major outstanding open questions.

Conjectures on $\boldsymbol{\Sigma}_{\boldsymbol{\lambda}} \equiv \boldsymbol{\sigma}\left(\boldsymbol{H}_{\boldsymbol{\omega}}(\lambda)\right)$ almost surely.

(1) Fix $\lambda>0$. $\exists E_{0}>2 d$ such that $\Sigma_{\lambda} \cap\left\{\left(-\infty,-E_{0}\right] \cup\left[E_{0}, \infty\right)\right\}$ is dense pure point with $2 d<E_{0}<2 d+\lambda$.

(2) $\exists \lambda_{0}$ such that $\lambda>\lambda_{0}, \Sigma$ is dense pure point.

(3) i. dimension $d=1, \Sigma_{\lambda}$ is always dense pure point.

ii. dimension $d=2, \Sigma_{\lambda}$ is always dense pure point.

iii. dimension $d \geq 3$, for small $\lambda \geq 0, \exists E_{m}=E_{m}(\lambda)<E_{0}$ of (1) such that $\Sigma_{\lambda} \cap\left[-E_{m}, E_{m}\right]$ is absolutely continuous.

We refer to Case 1 as spectral localization near the band edges, Case 2 as localization at large disorder. These are expected to hold independently of the dimension. Case 3 summarizes the dimension-dependent conjectures.

Statements (1), (2) and (3i) are known. This work on the lattice Anderson model is presented in the books of Carmona and Lacroix [21], and Pastur and Figotin [107]. In the multidimensional lattice case, the key original articles are those of Fröhlich and Spencer [56], Martinelli and Scoppola [102], Simon and Wolff [119], Kotani and Simon [94], Delyon, Soulliard, and [38], von Dreifus and Klein [132], and Aizenman and Molchanov [4].

Problems (3ii)-(3iii) remain open. Klein [88] proved the existence of extended states (a band of absolutely continuous spectrum near zero energy) for the Anderson model on the Bethe lattice. Another proof of this result was recently given by Froese, Hasler, and Spitzer [57], and a related stability result for the absolutely continuous spectrum was given by Aizenman, Sims, and Warzel [5]. The existence of extended states and a mobility edge $E_{m}$ for small $\lambda \geq 0$ for the lattice Anderson 
model, with decaying randomness, was also recently established by Kirsch, Krishna, and Obermeit [84]

1.5. Single Electron Models. There are several models of physical relevance which describe the behavior of a single electron in randomly perturbed media. We have the choice of the background operator $H_{0}$ and of the random potential $V_{\omega}$. The most well known situation is when the background operator $H_{0}=-\Delta+V_{p e r}$, where $V_{\text {per }}$ is a periodic function on $R^{d}$. This unperturbed operator describes a single electron moving in an infinite, periodic lattice. The basic random perturbation is the Anderson-type potential. It is described by a family of functions $u_{i}$, with $i \in \mathbb{Z}^{d}$, called the single-site potentials, and coupling constants $\left\{\omega_{i} \mid i \in \mathbb{Z}^{d}\right\}$. The potential is defined by

$$
V_{\omega}(x) \equiv \sum_{i \in \mathbb{Z}^{d}} \omega_{i} u_{i}(x)
$$

The family of coupling constants $\left\{\omega_{i} \mid i \in \mathbb{Z}^{d}\right\}$ forms a stochastic process on $\mathbb{Z}^{d}$. In the simplest model, the coupling constants are a collection of independent, identically distributed (iid) random variables. In the iid case, the random operators are ergodic (see chapter 2) if $u_{i}(x)=u(x-i)$, for all $i \in \mathbb{Z}^{d}$. More complicated models treat the case of correlations between the random variables (see, for example $[\mathbf{3 1}, \mathbf{1 3 2}]$. We can also introduce another family of vector-valued random variables $\left\{\xi_{i} \mid i \in \mathbb{Z}^{d}\right\}$ with $\xi_{i} \in B_{R}(0), 0<R<\frac{1}{2}$. We assume that the random variable $\xi_{i}$ has an absolutely continuous distribution, for example, a uniform distribution. These random variables will model thermal fluctuations of the scatterers with random strengths about the lattice points $\mathbb{Z}^{d}$. The random potential has the form

$$
V_{\omega, \omega^{\prime}}(x) \equiv \sum_{i \in \mathbb{Z}^{d}} \omega_{i} u_{i}\left(x-\xi_{i}\right) .
$$

The Anderson model on the lattice has been extensively studied. We refer to the books by Carmona and Lacroix [21] and by Pastur and Figotin [107] for the references. The basic papers, of relevance to the approaches described here for the continuous cases, include $[\mathbf{5 6}, \mathbf{7 6}, \mathbf{1 1 9}, \mathbf{1 2 0}, \mathbf{1 3 1}]$. There are now many results on localization at the bottom of the spectrum and at the band edges for the continuous Anderson model. These include $[\mathbf{8}, \mathbf{2 5}, \mathbf{9 0}, \mathbf{8 3}, \mathbf{8 6}, \mathbf{8 7}]$. These papers use the method of multiscale analysis explained in these lectures. Recently, the method of fractional moments was extended to continuum models and we refer the reader to $[\mathbf{3}, \mathbf{2 0}]$. The case of correlated random variables for lattice models is described in [132] and for the continuous models in [31].

In general, the background operator for electrons may have the form

$$
H_{0}=\left(p-A_{0}\right)^{2}+V_{0},
$$

where $p \equiv-i \nabla$, the vector-valued function $A_{0}$ is a reasonable vector potential and $V_{0}$ is a background potential, cf. [8]. The Landau Hamiltonian $H_{A}$ in two dimensions is a special case with a constant magnetic field $B$ generated by the vector potential $A=(B / 2)\left(-x_{2}, x_{1}\right)$, and $V_{0}=0$. Localization and the integrated density of states for this model have been extensively studied, see $[\mathbf{9}, \mathbf{2 6}, \mathbf{4 0}, \mathbf{4 1}$, $63,133,134]$, because of the role it plays in the theory of the integer quantum Hall effect (cf. [95]). 
There are other models of randomness of physical interest. We give a brief description of them here. In sections 3-5, we will concentrate on results for the Anderson-type potential, although they also hold for some of the other models.

1.5.1. Breather Model. Let $u>0$ be a single-site potential of compact support. We require a repulsive condition on $u:-x \cdot \nabla u(x) \geq 0$, and a relative boundedness condition on the Hessian of $u$. We assume that the random variables $\omega_{i}$ are iid with a common density supported some positive interval. The breather potential has the form

$$
V_{\omega}(x) \equiv \sum_{i \in \mathbb{Z}^{d}} u\left(\omega_{i}(x-i)\right) .
$$

One can show that the random family $H_{\omega}=-\Delta+V_{\omega}$ has an interval of localized states $\left[0, E_{1}\right]$, almost surely, for some $E_{1}>0[\mathbf{3 0}]$.

1.5.2. Random Displacement Model. The random potential for this model has the form

$$
V_{\omega}(x) \equiv \sum_{i \in \mathbb{Z}^{d}} u\left(x-i-\xi_{i}\right),
$$

where the vector-valued random variables $\left\{\xi_{i}(\omega)\right\}$ are distributed in a ball of radius $R<1 / 2$, as above. This model was studied in the case of $u \leq 0$ so that the single-site potential represents a potential well. Klopp [91] showed that in this case the model exhibits localization at negative energies provided the semiclassical parameter $h$, appearing in the Hamiltonian as $H(h)=-h^{2} \Delta+V_{\omega}$, is sufficiently small. Quantum tunneling plays a major role in the localization of states for this model. Localization in the general case is an open problem.

1.5.3. Poisson Model. One of the most realistic models of impurities randomly distributed in a perfect crystal is given by a Poisson potential. Let $X_{i}(\omega), i \in \mathbb{Z}$, represent the points of a Poisson process in $R^{d}$. Suppose that $u \geq 0$ is a single-site potential of compact support. The Poisson potential is given by

$$
V_{\omega}(x) \equiv \sum_{i \in \mathbb{Z}} u\left(x-X_{i}(\omega)\right) .
$$

Surprisingly, until recently, very little was known about these potentials except in one dimension. Stolz [125] recently proved that all states are localized for the onedimensional model. In arbitrary dimensions, Tip [127] has proved that for a class of repulsive potentials $u$, the integrated density of states is absolutely continuous at high energies. Most recently, Germinet, Klein and the author [59, 60, 61] proved Anderson and dynamical localization for this model. The work is related to recent work of Bourgain and Kenig [19] on the Anderson model with Bernoulli distributed random variables.

1.5.4. Gaussian Models. This random family of Schrödinger operators has the form (1.1), with $H_{0}=-\Delta$ and the random potential $V_{\omega}(x)$ a Gaussian process indexed by $\mathbb{R}^{d}$. This model has been recently studied $[\mathbf{5 2}, \mathbf{5 3}, \mathbf{5 4}]$. These authors prove a Wegner estimate, show the absence of absolutely continuous spectrum, and prove localization at negative energies.

1.6. Classical Wave Propagation. The localization of acoustic waves and of light is of theoretical and practical importance. Because of the absence of the electron-electron interaction, which might tend to obscure localization effects for electrons, it may be easier to detect the localization of light experimentally. For a 
review of these questions, we refer to $[\mathbf{1 3}, \mathbf{8 0}]$. The techniques used to prove bandedge localization for electrons can also be used for various models of classical waves propagating in inhomogeneous media. Some of the early work on localization for lattice models describing waves was done by Faris $[\mathbf{4 3}, \mathbf{4 4}]$ and Figotin and Klein [46]. We describe here the models studied in [33].

(1) Acoustic waves. The wave equation for acoustic waves propagating in a medium with sound speed $C$ and density $\rho$ is

$$
\partial_{t}^{2} \psi+\hat{H} \psi=0
$$

where the propagation operator $\hat{H}$ is given by

$$
\hat{H} \equiv-C^{2} \rho \nabla \rho^{-1} \nabla .
$$

By a standard unitary transformation, it suffices to consider the operator $H$, unitarily equivalent to $\hat{H}$, given by

$$
\begin{aligned}
H & \equiv-C \Delta C-\frac{1}{2}\left\{\frac{C^{2} \Delta \rho}{\rho}-\frac{3}{2} \frac{C^{2}|\nabla \rho|^{2}}{\rho^{2}}\right\} \\
& =-\left(C^{2} \rho\right)^{\frac{1}{2}} \nabla \cdot \rho^{-1} \nabla\left(C^{2} \rho\right)^{\frac{1}{2}},
\end{aligned}
$$

acting on the Hilbert space $\mathcal{H}=L^{2}\left(R^{d}\right), d \geq 1$. We consider perturbed sound speeds of the form

$$
C_{\omega}(g) \equiv\left(1+g \tilde{C_{\omega}}\right)^{-1 / 2} C_{0},
$$

for $g \geq 0$. To relate this to (1.2), we factor out the unperturbed sound speed $C_{0}$ and define the unperturbed acoustic wave propagation operator $H_{0}$ by

$$
H_{0} \equiv-C_{0} \rho^{\frac{1}{2}} \nabla \cdot \rho^{-1} \nabla \rho^{\frac{1}{2}} C_{0} .
$$

The coefficient $A_{\omega}$ appearing in (1.2) is given by

$$
A_{\omega} \equiv\left(1+g \tilde{C_{\omega}}\right) .
$$

(2) Electromagnetic waves. The wave equation for electromagnetic waves can be written in the form of equation (1.2) for vector-valued functions $\psi$. In this case, the operator $H$ describing the propagation of electromagnetic waves in a medium characterized by a dielectric function $\epsilon$ and a magnetic permeability $\mu=1$ is given by

$$
H \equiv-\epsilon^{-1 / 2} \Delta \Pi \epsilon^{-1 / 2},
$$

acting on the Hilbert space $\mathcal{H}=L^{2}\left(R^{3}, \mathbb{C}^{3}\right)$. The matrix-valued operator $\Pi$ is the orthogonal projection onto the subspace of transverse modes. We consider random perturbations of a background medium described by a dielectric function $\epsilon_{0}$ and given by

$$
\epsilon_{\omega}(g) \equiv 1+\epsilon_{0}+g \tilde{\epsilon_{\omega}},
$$

where $\tilde{\epsilon_{\omega}}$ is a stochastic process. The unperturbed operator describing the background medium is defined by

$$
H_{0} \equiv-\left(1+\epsilon_{0}\right)^{-1 / 2} \Delta \Pi\left(1+\epsilon_{0}\right)^{-1 / 2},
$$

and the coefficient $A_{\omega}$ in (1.2) is given by by a dielectric function $\epsilon_{0}$ and given by

$$
\epsilon_{\omega}(g) \equiv 1+\epsilon_{0}+g \tilde{\epsilon_{\omega}},
$$


where $\tilde{\epsilon_{\omega}}$ is a stochastic process. The unperturbed operator describing the background medium is defined by

$$
H_{0} \equiv-\left(1+\epsilon_{0}\right)^{-1 / 2} \Delta \Pi\left(1+\epsilon_{0}\right)^{-1 / 2},
$$

and the coefficient $A_{\omega}$ in (1.2) is given by

$$
A_{\omega} \equiv\left(1+g\left(1+\epsilon_{0}\right)^{-1} \tilde{\epsilon_{\omega}}\right) .
$$

We note that $A_{\omega}$ is the velocity of light for the realization $\omega$.

There have been several recent papers proving band-edge localization for these and related models. Figotin and Klein $[\mathbf{4 7}, \mathbf{4 8}]$ have results on band edge localization quite similar to ours. Stollmann $[\mathbf{1 2 1}]$ recently studied random perturbations of metrics. The random processes $\tilde{C_{\omega}}$ and $\tilde{\epsilon_{\omega}}$ can be of any of the type described in section 2 .

1.7. The Main Results on Band-Edge Localization. We now list our hypotheses necessary to prove localization at energies near the unperturbed bandedges $B_{ \pm}$. We will begin with conditions of the deterministic operator $H_{0}$. this operator, which describes the unperturbed media, is given in (1.19) in the Schrödinger case, (1.27) in the acoustic case, and in (1.31) in the Maxwell case. When it is necessary to distinguish these three cases, we will write $H_{0}^{X}$, with $X=S, A$, or $M$ for the Schrödinger acoustic, or Maxwell case, respectively.

(H1) The self-adjoint operator $H_{0}^{X}$ is essentially self-adjoint on $C_{0}^{\infty}\left(\mathbb{R}^{d}\right)$, for $X=S$ and $X=A$, and on $C_{0}^{\infty}\left(\mathbb{R}^{3}, \mathbb{C}^{3}\right)$ for $X=M$. The operator $H_{0}^{X}$ is semi-bounded and has an open spectral gap. That is, there exist constants $-\infty<-C_{0} \leq B_{-}<B_{+} \leq \infty$ so that

$$
\sigma\left(H_{0}\right) \subset\left(-C_{0}, B_{-}\right] \cup\left[B_{+}, \infty\right) .
$$

(H2) The operator $H_{0}^{X}$ is strongly locally compact in the sense that for any $f \in L^{\infty}\left(\mathbb{R}^{d}\right)$, for $X=S$ and $A$, or for any $f \in L^{\infty}\left(\mathbb{R}^{3}, \mathbb{C}^{3}\right)$ for $X=M$, with compact support, the operator $f\left(H_{0}\right)\left(H_{0}+C_{0}+1\right)^{-1} \in \mathcal{I}_{q}$, for some even integer $q$, with $1 \leq q<\infty$. Here, $\mathcal{I}_{q}$ denotes the $q^{t h}$-Schatten class, cf. [114].

(H3) Let $\rho(x) \equiv\left(1+\|x\|^{2}\right)^{1 / 2}$. The operator

$$
H_{0}(\alpha) \equiv e^{i \alpha \rho} H_{0} e^{-i \alpha \rho},
$$

defined for $\alpha \in \mathbb{R}$, admits an analytic continuation as a type-A analytic family to a strip

$$
S\left(\alpha_{0}\right) \equiv\left\{x+i y \in \mathbb{C}|| y \mid<\alpha_{0}\right\},
$$

for some $\alpha_{0}>0$.

Hypothesis (H1) is a condition on the unperturbed potential $V_{0}$ and the vector potential $A$ in the Schrödinger case, and on the unperturbed medium, as described by $C_{0}$ and $\epsilon_{0}$, for the classical wave case. Schrödinger operators with periodic potentials provide examples of operators $H_{0}$ with open spectral gaps, cf. [113]. As for classical waves, certain models of photonic crystals are known to have open gaps, cf. $[\mathbf{5 0}, \mathbf{5 1}, \mathbf{1 2 8}]$. Hypothesis $(\mathrm{H} 3)$ is satisfied for a large class of operators $H_{0}$, see $[\mathbf{8}, \mathbf{3 3}]$.

We now give hypotheses on the random potential given in (1.17)-(1.18) and in (1.28) and (1.34). The random potentials of Anderson-type are specified by giving conditions on the single-site potentials $u_{j}$, the coupling constants $\omega_{j}$, and 
the random variables $\xi_{j}$. We will use the following notation. We denote by $B_{r}(x)$ the ball of radius $r$ centered at $x \in \mathbb{R}^{d}$. A cube of side length $\ell$ centered at $x \in \mathbb{R}^{d}$ is denoted by $\Lambda_{\ell}(x)$. The characteristic function of a subset $A \subset \mathbb{R}^{d}$ is denoted by $\chi_{A}$.

(H4) The single-site potentials $u_{i} \geq C_{i} \chi_{B_{r_{i}}(0)}$ for some constant $C_{i}>0$ and some radius $r_{i}>0$. Furthermore, we assume that

$$
\sum_{j \in \mathbb{Z}^{d}}\left\{\int_{\Lambda_{1}(0)}\left|u_{j}(x)\right|^{p}\right\}^{1 / p}<\infty,
$$

for $p \geq d$ when $d \geq 2$ and $p=2$ when $d=1$.

We will assume that the random variables $\omega_{j}$, appearing in the Andersontype potential (1.17), form a stationary stochastic process indexed by $\mathbb{Z}^{d}$. The probability space for this process is $\Omega=[-m, M]^{\mathbb{Z}^{d}}$, for some constants $m \neq M$ and $0 \leq m, M \leq \infty$. In the case that the range of the random variables is unbounded, we will need to control some of the moments of $\omega_{j}$.

(H5) The random variables $\omega_{j}$ have $p$ finite moments:

$$
\mathbb{E}\left\{\omega_{j}^{k}\right\}<\infty, \quad k=1,2, \ldots, p,
$$

where $p$ is the dimension-dependent constant given in (H4).

We refer to the review article of Kirsch [82] for a proof of the fact that hypotheses (H4)-(H5) imply the essential self-adjointness of $H_{\omega}$ on $C_{0}^{\infty}\left(\mathbb{R}^{d}\right)$.

(H6) The conditional probability distribution of $\omega_{0}$, conditioned on $\omega_{0}{ }^{\perp} \equiv$ $\left\{\omega_{i} \mid i \neq 0\right\}$, is absolutely continuous with respect to Lebesgue measure.

It has a density $h_{0}$ satisfying $\left\|h_{0}\right\|_{\infty}<\infty$.

Hypothesis (H5) implies that the correlation function $C(i, j) \equiv \mathbb{E}\left\{\omega_{i} \omega_{j}\right\}-$ $\mathbb{E}\left\{\omega_{i}\right\} \mathbb{E}\left\{\omega_{j}\right\}$ exists and is finite. An example of a process satisfying (H5) is a Gaussian process on $\mathbb{Z}^{d}$ with each local covariance function $C(i, j), i, j \in \Lambda$ being a bounded, invertible matrix. In the case that the random coupling constants are iid random variables, hypothesis (H6) reduces to the usual assumption that a density exits as a bounded, compactly-supported function. For simplicity, in the remainder of these notes, we will usually assume that $h_{0}$ of $(\mathrm{H} 6)$ is compactly-supported. This automatically implies that (H5) is satisfied.

(H7) The density $h_{0}$ decays sufficiently rapidly near $-m$ and near $M$ in the following sense:

$$
\begin{aligned}
& 0<\mathbb{P}\{|\omega+m|<\varepsilon\} \leq \varepsilon^{3 d / 2+\beta}, \\
& 0<\mathbb{P}\{|\omega-M|<\varepsilon\} \leq \varepsilon^{3 d / 2+\beta},
\end{aligned}
$$

for some $\beta>0$.

Recent work of Klopp [92] on the existence of internal Lifshitz tails may allow us to remove hypothesis (H7).

We need to assume the existence of deterministic spectrum $\Sigma$ for families of randomly perturbed operators as in (1.1)-(1.2) with $H_{0}$ satisfying (H1)-(H7). If $H_{0}$ is periodic with respect to the translation group $\mathbb{Z}^{d}$, and for Anderson-type perturbations described above, the random families of operators $H_{\omega}$ are measurable, 
self-adjoint, and $\mathbb{Z}^{d}$-ergodic. In this case, it is known (cf. $\left.[\mathbf{2 1}, \mathbf{1 0 7}]\right)$ that the spectrum of the family is deterministic.

(H8) There exists constants $B_{ \pm}^{\prime}$ satisfying $B_{-}<B_{-}^{\prime}<B_{+}^{\prime}<B_{+}$such that

$$
\Sigma \cap\left\{\left(B_{-}, B_{-}^{\prime}\right) \cup\left(B_{+}^{\prime}, B_{+}\right)\right\} \neq \emptyset \text {, and } \Sigma \cap\left(B_{-}^{\prime}, B_{+}^{\prime}\right)=\emptyset .
$$

In light of hypothesis (H8), we define the band edges of the almost sure spectrum $\Sigma$ near the gap $G$, as follows:

$$
\tilde{B}_{-} \equiv \sup \left\{E \leq B_{-}^{\prime} \mid E \in \Sigma\right\},
$$

and

$$
\tilde{B}_{+} \equiv \inf \left\{E \geq B_{+}^{\prime} \mid E \in \Sigma\right\},
$$

Examples of operators satisfying these conditions may be found in $[\mathbf{8}, \mathbf{3 3}, \mathbf{4 7}$, 48]. The main results are the following two theorems. We refer to the first theorem as Band-Gap Localization.

TheOREM 1.2. Assume (H1) - (H8). There exist constants $E_{ \pm}$satisfying $B_{-} \leq E_{-}<\tilde{B}_{-}$and $\tilde{B}_{+}<E_{+} \leq B_{+}$such that $\Sigma \cap\left(E_{-}, E_{+}\right)$is pure point with exponentially decaying eigenfunctions. The eigenvalues have finite multiplicity almost surely.

For lattice models, the eigenvalues in the localization regime are known to be simple (multiplicity one), cf. $[\mathbf{8 9}, \mathbf{1 1 6}]$. The finite multiplicity of the eigenvalues for the continuum models is an open question, although the finiteness of the multiplicity is proved in [25], as discussed in section 3.4, and another proof is given in [65]. One can also establish dynamical localization for the regions of $\Sigma$ where Theorem 1.2 holds. We refer the reader to $[\mathbf{5 8}, \mathbf{6 2}]$.

We are able to prove a strong result on the IDS using hypotheses that are weaker than (H1)-(H8). In chapter 4, we list a weaker set of hypotheses (A1)-(A4) that are necessary to prove the following theorem. The full strength of the result on the IDS is given in [28].

TheOREM 1.3. Assume (A1) - (A4) of chapter 4. The integrated density of states is locally uniformly Lipschitz continuous on $\mathbb{R}$.

1.8. Description of the Contents: A Road Map to Localization. In order to give the reader a global picture of the theory of localization for continuous, random systems, we give an outline of the proof of localization for additively and for multiplicatively perturbed operators. This will serve as a guide to the contents of the chapters of these notes.

We use the fixed-energy approach (see Figotin and Klein [45], von Dreifus and Klein [131], and Germinet and Klein [62], for the energy-interval approach) which uses spectral averaging and Kotani's trick. The arguments apply to both additive and multiplicative perturbations.

a. The first goal is to prove almost sure exponential decay of the resolvent of the Hamiltonian $H_{\omega}$ for Lebesgue almost energies in an interval $I_{\delta} \equiv$ $\left[\tilde{B}_{-}-\delta / 2, \tilde{B}_{-}\right] \cup\left[\tilde{B}_{+}, \tilde{B}_{+}+\delta / 2\right]$, for a fixed $\delta>0$, near the edges of the spectral gap of $\Sigma$. This estimate has the form

$$
\mathbb{P}\left\{\sup _{\epsilon>0}\left\|\chi_{x} R_{\omega}(E+i \epsilon) \chi_{y}\right\| \leq e^{-m_{0}|x-y|}\right\}=1,
$$


for a constant $m_{0}>0$, depending on the interval $I_{\delta}$, and for all $x, y \in$ $\mathbb{R}^{d}$ such that $\|x-y\|$ is sufficiently large. The functions $\chi_{x}$ and $\chi_{y}$ are compactly supported in a small neighborhood of $x$ and $y$, respectively.

To prove this estimate, we need to establish two estimates on finite-volume Hamiltonians. These Hamiltonians, $H_{\Lambda}$, for bounded regular regions $\Lambda \subset \mathbb{R}^{d}$, are local perturbations of the background operator $H_{0}$. As such, they have only discrete spectra in the unperturbed spectral gap $G$. We must control, with a good probability depending on $|\Lambda|$, the location of these eigenvalues. This is done in chapters 4 and 5 . First, in chapter 4 , we prove a Wegner estimate which states that for any energy $E \in G$, and for any $\eta>0$ such that $[E-\eta, E+\eta] \subset G$,

$$
\mathbb{P}\left\{\operatorname{dist}\left(\sigma\left(H_{\Lambda}\right), E\right) \leq \eta\right\} \leq C_{W} \eta|\Lambda| .
$$

Second, we show in chapter 5 that there exists $\delta_{\Lambda}>0$ such that

$$
\sigma\left(H_{\Lambda}\right) \cap\left[\tilde{B}_{-}-\delta_{\Lambda}, \tilde{B}_{-}\right] \cup\left[\tilde{B}_{+}, \tilde{B}_{+}+\delta_{\Lambda}\right]=\emptyset,
$$

with a probability which tends to 1 as $|\Lambda| \rightarrow \infty$. Using a refined Combes-Thomas estimate, which is proven in $[8]$, we prove that this result implies that the resolvent of the local Hamiltonian $H_{\Lambda}$ decays exponentially at all energies in the interval $I_{\delta} \equiv\left[\tilde{B}_{-}-\delta_{\Lambda} / 2, \tilde{B}_{-}\right] \cup\left[\tilde{B}_{+}, \tilde{B}_{+}+\delta_{\Lambda} / 2\right]$, with the same probability, provided $|\Lambda|$ is large enough.

These two estimates for the finite-volume Hamiltonians $H_{\Lambda}$ are the starting point of a multiscale analysis as developed in [25]. We present this analysis in chapters 5 and 9 , with the modifications of this analysis necessary for multiplicatively-perturbed operators given in chapter 8 . This leads directly to the fixed-energy exponential decay result stated in (1.19) for Lebesgue almost-every energy in $I_{\delta}=\left[\tilde{B}_{-}-\delta / 2, \tilde{B}_{-}\right] \cup\left[\tilde{B}_{+}, \tilde{B}_{+}+\delta / 2\right]$, for an appropriate $\delta=\delta_{\Lambda_{0}}$, where $\Lambda_{0}$ is sufficiently large.

b. The second main step is to extend this fixed-energy result to prove localization in the interval $I_{\delta}$. To do this, we need a result which is referred to as spectral averaging (this result is also used in the proof of the Wegner estimate presented in chapter 4). The main consequence of this technical result states, roughly speaking, that the expectation of a spectral measure associated with the random family $H_{\omega}$ is absolutely continuous with respect to Lebesgue measure. This is often referred to as Kotani's trick in the literature. This trick allows us to transfer information about the exponential decay of the resolvent at Lebesgue almost every energy in $I_{\delta}$ to almost every energy with respect to the spectral measure for $H_{\omega}$ for almost every $\omega$. We prove these results for additive perturbations in chapter 3 and present the modifications necessary for multiplicative perturbations. This result, together with the fixed energy resolvent estimates discussed above, are then combined with some probabilistic arguments of [25] to prove that $\Sigma \cap\left\{\left[\tilde{B}_{-}-\delta / 2, \tilde{B}_{-}\right] \cup\left[\tilde{B}_{+}, \tilde{B}_{+}+\delta / 2\right]\right.$ is pure point. This established localization. A separate argument as in $[\mathbf{2 5}]$ proves that the eigenfunctions decay exponentially.

c. One can also prove the absence of diffusion, in the sense that

$$
\limsup _{t \rightarrow \infty} t^{-1}\left\langle x^{2}(t)\right\rangle_{\psi}=0
$$


using an argument of Barbaroux [11]. In fact, recent work of DeBièvre and Germinet [58] (see also [62]) establish dynamical localization for the models discussed in these notes.

Acknowledgments. I thank Carlos Villegas-Blas for the invitation to lecture at the IV Escuela de Verano en Análisis y Fisica Matemática at the Instituto de Matemáticas, Cuernavaca, Mexico, in May 2005, and for all his support. The basic results presented in these notes were obtained in collaboration with Jean-Michel Combes, whom I warmly thank for enjoyable collaborations. I also thank the many colleagues who contributed to my understanding of the topics discussed in these notes, including Michael Aizenman, Jean-Marie Barbaroux, Jean Bellissard, Stephan DeBièvre, Francois Germinet, Werner Kirsch, Abel Klein, Frédéric Klopp, Hajo Leschke, Eric Mourre, Peter Müller, Shu Nakamura, Peter Stollmann, Gunter Stolz, and Adriaan Tip.

\section{Basic Results in the Theory of Random Operators}

2.1. Introduction. We give a review of certain aspects of the theory of random operators that will be used in this book. Extensive discussions of these topics can be found in the books of Carmona and Lacroix [21], Pastur and Figotin [107], Stollmann [123], and the review article of Kirsch [82]. In the first part, we review the questions of self-adjointness and the deterministic spectrum. We are mostly interested in techniques of computing the deterministic spectrum. The second part of this chapter is devoted to the integrated density of states (IDS). We introduce the notion of finite-volume operators that will figure significantly in the other chapters.

2.2. A Summary of Basic Results on Self-Adjoint Operators. The topics of self-adjointness, deterministic spectrum, and computation of the deterministic spectrum are discussed in this section.

The random families of Schrödinger operators $\left\{H_{\omega} \mid \omega \in \Omega\right\}$ that we deal with are all symmetric operators on the dense domain $C_{0}^{\infty}\left(\mathbb{R}^{d}\right) \subset L^{2}\left(\mathbb{R}^{d}\right)$, for almost every realization $\omega \in \Omega$. We will not treat the most general situations of selfadjointness for $H_{\omega}$, but will concentrate on certain models. An extensive discussion of self-adjointness for Schrödinger operators can be found in $[\mathbf{1 1 1}, \mathbf{1 1 3}]$.

We are interested in the stability of self-adjointness under perturbations. The unperturbed operator for Schrödinger operators is the Laplacian $-\Delta$ on $L^{2}\left(\mathbb{R}^{d}\right)$, which is self-adjoint on the domain $H^{2}\left(\mathbb{R}^{d}\right)$. Let us recall four basic topics concerning self-adjointness for linear operators, and its stability under perturbations: 1) relative-boundedness results, and 2) results for positive potentials, 3) results applicable to Schrödinger operators with magnetic and electric fields, and 4) stability of self-adjointness under multiplicative perturbations. This latter part is applicable to the study of classical waves.

\section{Relatively-Bounded Perturbations}

We will first review relatively-bounded operators. Let $A$ be a closed operator on a Hilbert space $\mathcal{H}$. We consider perturbations of $A$ by a class of linear operators $B$ on the Hilbert space $\mathcal{H}$.

Definition 2.1. An operator $B$ is called $A$-bounded if $D(B) \supset D(A)$.

It is obvious, but important to note, that any bounded operator $B \in \mathcal{L}(\mathcal{H})$ is $A$-bounded for any closed linear operator $A$. Relative-boundedness implies an important boundedness relation between the operators. 
Proposition 2.1. If $\rho(A) \neq \phi$ and $B$ is $A$-bounded, then there exist nonnegative constants $a$ and $b$ such that

$$
\|B u\| \leq a\|A u\|+b\|u\|,
$$

for all $u \in D(A)$.

Let us recall that the smallest nonnegative constant $a$ for which (2.1) holds for all $u \in D(A)$ is called the relative A-bound of $B$. The fundamental result on the stability of self-adjointness under relatively-bounded perturbations is the KatoRellich Theorem.

TheOREM 2.2. (Kato-Rellich Theorem). Let $A$ be self-adjoint and let $B$ be a symmetric and $A$-bounded operator with relative $A$-bound less than one. Then $A+B$ is self-adjoint on $D(A)$.

The basic application of this result to Schrödinger operators is the following theorem.

THEOREM 2.3. Let the real-valued function $V \in L^{p}\left(\mathbb{R}^{d}\right)+L^{\infty}\left(\mathbb{R}^{d}\right)$. Then the operator $H_{V} \equiv-\Delta+V$, defined on $D(\Delta)=H^{2}\left(\mathbb{R}^{d}\right)$, is self-adjoint if $p \geq d / 2$ for $d \geq 5$, if $p>2$, for $d=4$, and if $p \geq 2$ for $d \leq 3$. Furthermore, $H_{V}$ is essentially self-adjoint on $C_{0}\left(\mathbb{R}^{d}\right)$ under these conditions.

Note that the conditions on $V$ in Theorem 2.3 allow two types of behavior at infinity. If $V$ decays at infinity like $\|x\|^{\alpha}$, for $\alpha>d / p$, then $H_{V}$ is self-adjoint on the domain $H^{2}\left(\mathbb{R}^{d}\right)$. At the other extreme, if $V$ is everywhere bounded, then $H_{V}$ is self-adjoint on the domain $H^{2}\left(\mathbb{R}^{d}\right)$. This will be the case for most random potentials of Anderson-type.

Positive Potentials

When the potential $V$ is nonnegative and not-necessarily bounded, self-adjointness can be established using the Kato inequality. We will first state this inequality for the Laplacian on $\mathbb{R}^{d}$.

TheOREM 2.4. Let $u \in L_{l o c}^{1}\left(\mathbb{R}^{n}\right)$ and suppose that the distributional Laplacian $\Delta u \in L_{l o c}^{1}\left(\mathbb{R}^{n}\right)$. Then

$$
\Delta|u| \geq \operatorname{Re}[(\operatorname{sgn} u) \Delta u],
$$

in the distributional sense.

We now consider a real potential $V \in L_{l o c}^{2}\left(\mathbb{R}^{d}\right)$. We define $H_{V}=-\Delta+V$ on $D\left(H_{V}\right) \equiv D(\Delta) \cap D(V)$, where $D(\Delta)=H^{2}\left(\mathbb{R}^{n}\right)$ and

$$
D(V)=\left\{\left.f \in L^{2}\left(\mathbb{R}^{n}\right)\left|\int\right| V f\right|^{2}<\infty\right\} .
$$

Note that $C_{0}^{\infty}\left(\mathbb{R}^{n}\right) \subset D\left(H_{V}\right)$, so $H_{V}$ is densely defined.

Theorem 2.5. Let $V \in L_{l o c}^{2}\left(\mathbb{R}^{d}\right)$ and $V \geq 0$. Then, the Schrödinger operator $H_{V}=-\Delta+V$ is essentially self-adjoint on $C_{0}^{\infty}\left(\mathbb{R}^{d}\right)$.

\section{Electric and Magnetic Fields}

We will consider as our background operators $H_{0}$ Schrödinger operators with electric and magnetic potentials. Let $A$ be a $C^{1}$-vector potential on $\mathbb{R}^{d}$. The pure magnetic Schrödinger operator is defined by $H_{A} \equiv(-i \nabla-A)^{2}$. We will treat the special two-dimensional case of a constant magnetic field in detail in chapter 7 . A 
vector potential in two dimensions $\left(x_{1}, x_{2}\right)$ which describes a constant magnetic field of strength $B>0$ in the perpendicular $x_{3}$-direction is given by

$$
A=\frac{B}{2}\left(x_{2},-x_{1}\right) \text {. }
$$

The choice of a vector potential $A$ for a given magnetic field is not unique since the transformation $A \rightarrow A+\nabla \phi$ results in the same magnetic field. A consequence of this is the gauge invariance of the magnetic Schrödinger operator $H_{A}$. For a real-valued differentiable function $\phi$, we have the identity:

$$
e^{-i \phi} H_{A+\nabla \phi} e^{i \phi}=H_{A} \text {. }
$$

In the presence of an external potential $V$, the Hamiltonian $H_{V, A}$ has the form

$$
H_{V, A}=(p-A)^{2}+V,
$$

where $p \equiv-i \nabla$. The basic self-adjointness theorem is due to Leinfelder and Simader [99]. In order to state this theorem, we need to define the Kato class of potentials $K_{d}\left(\mathbb{R}^{d}\right)$.

Definition 2.6. A real-valued potential $V \in K_{d}\left(\mathbb{R}^{d}\right)$, for $d \geq 3$ if

$$
\lim _{\alpha \rightarrow 0}\left\{\sup _{x} \int_{\|x-y\| \leq \alpha}\|x-y\|^{2-d}|V(y)| d^{d} y\right\}=0,
$$

and for $d=2$ if the same identity holds with the kernel $\|x-y\|^{2-d}$ replaced by $\log \|x-y\|$.

TheOREM 2.7. Let $V \in L_{l o c}^{2}\left(\mathbb{R}^{d}\right), V_{-} \in K_{d}\left(\mathbb{R}^{d}\right)$, and the vector potential $A \in L_{l o c}^{4}\left(\mathbb{R}^{d}\right)^{d}$. The Hamiltonian $H_{V, A}=(p-A)^{2}+V$ is essentially self-adjoint on $C_{0}^{\infty}\left(\mathbb{R}^{d}\right)$.

The Schrödinger operator describing a particle in a constant external electric field $E$ and potential $V$ is given by

$$
H_{V, E} \equiv-\Delta+x \cdot E+V .
$$

Because the potential $x \cdot E$ is unbounded, self-adjointness of the Hamiltonian $H_{0, E}$ requires a separate treatment. The fundamental theorem is due to Faris and Lavine. Rather than cite the theorem in complete generality (cf. [111]), we give a version which covers (2.7).

THEOREM 2.8. Let $V$ be a real-valued potential in $L^{p}\left(\mathbb{R}^{d}\right)$, where $p \geq 2$ if $d \leq 3, p>2$ if $d=4$, or $p \geq d / 2$ if $d \geq 5$. Then the Stark Hamiltonian $H_{V, E} \equiv$ $-\Delta+x \cdot E+V$ is essentially self-adjoint on $C_{0}^{\infty}\left(\mathbb{R}^{d}\right)$.

2.3. Basic Results on Families of Random Operators. The basic techniques for proving essential self-adjointness of a random family on $C_{0}^{\infty}\left(\mathbb{R}^{d}\right)$, for almost every $\omega$, is to prove that the random potential $V_{\omega}$ satisfies the conditions of one of the above theorems with probability one. The Borel-Cantelli Lemmas are quite useful for establishing this.

Since a random family $\left\{H_{\omega} \mid \omega \in \Omega\right\}$ of self-adjoint operators consists, in general, of uncountably-many self-adjoint operators, the study of the spectrum of any individual operator $H_{\omega}$ may not shed light on the properties of the family. An illuminating example is given in Chapter 1 , section 1.4, for which the Hamiltonian is a pure random potential $H_{\omega}=V_{\omega}$ on $\ell\left(\mathbb{Z}^{d}\right)$. Let us suppose that the iid (independent, identically distributed) random variables take values in $[0,1]$ with a 
uniform distribution. For any fixed valued of $\omega$, the operator $V_{\omega}$ has pure point spectrum consisting of the closure of the set $\left\{\omega_{n} \mid n \in \mathbb{Z}^{d}\right\}$. Note that this set changes enormously as $\omega$ varies. For example, if $\omega_{n}=\lambda \in[0,1]$, for every $n \in \mathbb{Z}^{d}$, then the operator has a single, infinitely degenerate eigenvalue $\lambda$ with each localized delta function $\delta_{n, m}$, for $n$ fixed, an $\ell^{2}$-eigenvector. On the other hand, suppose that $\phi: \mathbb{Z}^{d} \rightarrow[0,1] \cap Q$ is a bijection. If we take a representation $H_{\omega}$ for which $\omega_{n}=\phi(n)$, then the spectrum of $H_{\omega}$ is the closed interval [0,1], the range of the potential $V_{\omega}$. In this case, the spectrum consists of only eigenvalues, and is an example of dense pure point spectrum. Clearly, the spectrum of an individual $H_{\omega}$ varies greatly as the realization $\omega$ changes. We would like to find a set, which we call the deterministic spectrum which is the spectrum of the operators $H_{\omega}$, for almost every $\omega$.

Pastur [106] seems to have been the first to realize that the family $\left\{H_{\omega}\right\}$ has a notion of spectrum associated with it provided it is ergodic in a certain sense. We will not go into the general theory here. The probability spaces $\Omega$ of interest to us are product spaces of the form $\Pi_{n \in \mathbb{Z}^{d}} J$, where $J \subset \mathbb{R}$ is the support of the probability distribution.

Definition 2.9. Let $(\Omega, \mathbb{P})$ be a complete probability space. We suppose that the family of maps $\left\{\alpha_{x}: \Omega \rightarrow \Omega \mid x \in I\right\}$ is a subgroup of the automorphism of $(\Omega, \mathbb{P})$. We say that it acts ergodically on $(\Omega, \mathbb{P})$ if 1$)$ it is measure-preserving, that is, $\mathbb{P}\left(\alpha_{x}^{-1}(A)\right)=\mathbb{P}(A)$, for all measurable subsets $A \subset \Omega$ and $x \in I$, and, 2) if for any measurable subset $A \subset \Omega$, the condition $\alpha_{x}(A)=A$, for all $x \in I$, implies either $\mathbb{P}(A)=1$ or $\mathbb{P}(A)=0$.

In all cases of interest, the index set $I$ will be the additive group $\mathbb{Z}^{d}$ or $\mathbb{R}^{d}$. We note that the family $\left\{\alpha_{x}: \Omega \rightarrow \Omega \mid x \in I\right\}$ need only form a semigroup. Given that our measure spaces are products, the maps $\alpha_{x}$ are translations. In fact, by using the canonical representation of a stochastic process, we can always represent these maps $\alpha_{x}$ by translations. These translations induce a natural action on $L^{2}\left(\mathbb{R}^{d}\right)$ or $\ell^{2}\left(\mathbb{Z}^{d}\right)$ by unitary operators,

$$
U_{x} f(y)=f(y-x) .
$$

We are interested in the quantity $U_{x} H_{\omega} U_{x}^{-1}$. Before we give the main definition, we clarify the notion of measurability for self-adjoint operators.

Definition 2.10. A family of self-adjoint operators $\left\{H_{\omega} \mid \omega \in \Omega\right\}$ is called measurable if the functions $\left\langle\phi,\left(H_{\omega}-z\right)^{-1} \psi\right\rangle$, for any $\phi, \psi \in \mathcal{H}$, are measurable for some $z$, with $\operatorname{Im}(z) \neq 0$.

Definition 2.11. A measurable family of self-adjoint operators $\left\{H_{\omega}\right\}$ is ergodic with respect to an ergodic action of the translations $\left\{\alpha_{x}: \Omega \rightarrow \Omega \mid x \in I\right\}$ if it satisfies the covariance identity

$$
H_{\alpha_{x}^{-1}(\omega)}=U_{x} H_{\omega} U_{x}^{-1}
$$

for all $x \in I$.

To simplify terminology, we will call a measurable, ergodic family of self-adjoint operators simply an ergodic family of self-adjoint operators. The fundamental result is the following theorem. 
Theorem 2.12. (Pastur's Theorem [106].) Let $\left\{H_{\omega} \mid \omega \in \Omega\right\}$ be an ergodic family of self-adjoint operators. Then, there exists a set $\Sigma \subset \mathbb{R}$ such that $\Sigma=$ $\sigma\left(H_{\omega}\right) \mathbb{P}$-almost surely.

Another way to phrase this result is the following. There exists a set $\Omega_{1} \subset \Omega$, with $\mathbb{P}\left(\Omega_{1}\right)=1$, so that $\sigma\left(H_{\omega}\right)=\Sigma$, for all $\omega \in \Omega_{1}$. We will call the set $\Sigma$ the deterministic spectrum of the family of self-adjoint operators.

The spectrum of any self-adjoint operator has a natural decomposition into three components consisting of the pure point, absolutely continuous, and singular continuous spectra. It is natural to ask if the deterministic spectrum has a similar, deterministic decomposition. This result was proved by Kunz and Soulliard [97], and by Kirsch and Martinelli [85].

THEOREM 2.13. Let $\left\{H_{\omega} \mid \omega \in \Omega\right\}$ be an ergodic family of self-adjoint operators. Then, there exist sets $\Sigma_{p p}, \Sigma_{a c}$, and $\Sigma_{s c}$, and a set $\Omega_{1} \subset \Omega$, with $\mathbb{P}\left(\Omega_{1}\right)=1$, such that

$$
\begin{aligned}
& \sigma_{p p}\left(H_{\omega}\right)=\Sigma_{p p} \forall \omega \in \Omega_{1}, \\
& \sigma_{p p}\left(H_{\omega}\right)=\Sigma_{p p} \forall \omega \in \Omega_{1}, \\
& \sigma_{p p}\left(H_{\omega}\right)=\Sigma_{p p} \forall \omega \in \Omega_{1} .
\end{aligned}
$$

2.3.1. Techniques for Computing the Deterministic Spectrum. We mention two methods commonly used for computing the deterministic spectrum of ergodic families of self-adjoint operators. We will use these in the later chapters.

Sets of Positive Probability Measure. The most immediate consequence of Theorem 2.13 is the following approach to computing the deterministic spectrum $\Sigma$ for an ergodic family of self-adjoint operators $\left\{H_{\omega} \mid \omega \in \Omega\right\}$. Suppose that a set $I \subset \sigma\left(H_{\omega}\right)$, for $\omega \in \Omega_{0} \subset \Omega$, and that $\mathbb{P}\left(\Omega_{0}\right)>0$. Then, it follows that $I \subset \Sigma$. It is often easy to construct such a set of configurations $\Omega_{0}$.

Determining Configurations and Periodic Approximations. A second approach to the computation of $\Sigma$ consists of approximating the random potential $V_{\omega}$ by periodic operators. This result follows from a more general one on the deterministic spectrum. A base for the probability space $(\Omega, I P)$ is a measurable subset $\Omega_{0} \subset \Omega$ such that i) $\left.\mathbb{P}\left(\Omega_{0}\right)=1,2\right)$ the operator $H_{\omega}$ is self-adjoint for any $\omega \in \Omega_{0}$, and 3) the map $\omega \in \Omega_{0} \rightarrow H_{\omega}$ is strong resolvent continuous. This means that for any sequence $\omega_{n} \rightarrow \omega$ in $\Omega_{0}$, we have $\left\|\left\{\left(H_{\omega_{n}}-z\right)^{-1}-\left(H_{\omega}-z\right)^{-1}\right\} \phi\right\|=0$, for any $\phi \in \mathcal{H}$.

THEOREM 2.14. Let $\left\{H_{\omega} \mid \omega \in \Omega\right\}$ be an ergodic family of self-adjoint operators with base $\Omega_{0}$. If $\Omega_{1} \subset \Omega_{0}$ is dense in the support of the probability measure $\mathbb{P}$, then

$$
\Sigma=\overline{\cup_{\omega \in \Omega_{1}} \sigma\left(H_{\omega}\right)} .
$$

The interesting aspect of this theorem is that it might very well be that $\mathbb{P}\left(\Omega_{1}\right)=0$. For the Anderson model with a continuous density $g$, it can be shown that $\Omega_{1} \equiv\left\{\omega \mid V_{\omega}\right.$ is periodic $\}$ is dense in the support of the probability measure. Hence, the deterministic spectrum can be computed from the closure of the union of the spectrum of all periodic realizations of the potential.

2.4. The Integrated Density of States. For a quantum mechanical system described by a Hamiltonian $H$, the density of states (DOS) is a measure of the average number of states of the system per unit volume. It is defined through a 
limiting process as the volume of the system becomes infinite. This limiting process is called the thermodynamic limit. To describe it, we denote by $\Lambda_{\ell}(x)$ the cube of side length $\ell$ centered at the point $x \in \mathbb{R}^{d}$,

$$
\Lambda_{\ell}(x) \equiv\left\{y \in \mathbb{R}^{d}|| x_{i}-y_{i} \mid<\ell / 2, i=1, \ldots, d\right\} .
$$

We will denote an arbitrary, bounded, open, simply connected subset of $\mathbb{R}^{d}$ by $\Lambda$.

The DOS is well-defined for a system in a finite volume region $\Lambda$. Let $H_{\Lambda}^{X}$ be defined as the self-adjoint extension of $H \mid C_{0}^{\infty}(\Lambda)$, with boundary conditions $X$ imposed on $\partial \Lambda$, the boundary of $\Lambda$. We will work with Dirichlet boundary conditions $(X=D)$, and Neumann boundary conditions $(X=N)$. We assume that the spectrum is semi-bounded below, although more general situations can be treated. For regular regions $\Lambda$, the operators $H_{\Lambda}^{X}$ have only discrete spectrum, which we denote by $\left\{E_{j}(\Lambda)\right\}$. The set of eigenvalues is ordered so that $-\infty<E_{1}(\Lambda)<E_{2}(\Lambda) \leq E_{3}(\Lambda), \ldots$, where we repeat an eigenvalue according to its multiplicity. We define the finite-volume integrated density of states for the system in the region $\Lambda$ by

$$
N_{\Lambda}^{X}(E) \equiv \frac{1}{|\Lambda|} \#\left\{j \mid E_{j}(\Lambda) \leq E, \text { which includes multiplicities }\right\} .
$$

It is useful, for later purposes, to write these expressions using the spectral projectors of $H_{\Lambda}^{X}$. For any $I \subset \mathbb{R}$, let $E_{\Lambda}^{X}(I)$ denote the spectral projector for $H_{\Lambda}^{X}$ and the interval $I$. We denote by $\operatorname{Tr}_{\Lambda}$ the trace on the Hilbert space $L^{2}(\Lambda)$. Then, we have

$$
N_{\Lambda}^{X}(I)=|\Lambda|^{-1} \operatorname{Tr}_{\Lambda}\left(E_{\Lambda}^{X}(I)\right) .
$$

Because the spectrum of $H_{\Lambda}^{X}$ is discrete, the corresponding density of states defines a pure point measure, called the finite-volume density of states measure. It is given by

$$
d N_{\Lambda}^{X}(E) \equiv \frac{1}{|\Lambda|} \sum_{j} \delta\left(E-E_{j}(\Lambda)\right) d E .
$$

The finite-volume integrated density of states $N_{\Lambda}^{X}(E)$ is the distribution function corresponding to this measure,

$$
N_{\Lambda}^{X}(E)=\int_{-\infty}^{E} d N_{\Lambda}^{X}(\lambda)
$$

We now pass to the thermodynamic limit of $N_{\Lambda}^{X}(E)$. Formally, the integrated density of states (IDS) $N^{X}(E)$, is defined by

$$
N^{X}(E)=\lim _{\Lambda \rightarrow \mathbb{R}^{d}} N_{\Lambda}^{X}(E)
$$

whenever the limit exits. If the IDS exists, as defined in (2.16), then the DOS measure is defined in the usual way starting with the measure of an interval $I=\left[I_{-}, I_{+}\right]$, for which $N^{X}(I) \equiv N^{X}\left(I_{+}\right)-N^{X}\left(I_{-}\right)$, provided these are points of continuity for $N^{X}(E)$. Alternatively, the DOS measure is the vague limit of the finite-volume DOS measures defined in (2.14). That is, for any $f \in C_{0}^{\infty}\left(\mathbb{R}^{d}\right)$, we have,

$$
\int f(E) d N^{X}(E)=\lim _{\Lambda \rightarrow \mathbb{R}^{d}} \int f(E) d N_{\Lambda}^{X}(E) .
$$


It is easy to compute the high energy asymptotics of IDS for the Laplacian on $\mathbb{R}^{d}$. For either boundary condition, one finds that

$$
N_{\Lambda}^{X}(E) \sim E^{d / 2} .
$$

For an elliptic operator of second order, like a Schrödinger operator with a locally bounded potential, the Weyl estimate of the asymptotic distribution of eigenvalues guarantees that

$$
N_{\Lambda}^{X}(E) \leq \frac{\omega_{d} E^{d / 2}}{(2 \pi)^{d / 2}},
$$

where $\omega_{d}$ is the volume of the unit ball in $d$-dimensions so that

$$
\limsup _{\Lambda \rightarrow \mathbb{R}^{d}} N_{\Lambda}^{X}(E)
$$

always exists.

2.4.1. Sketch of the Existence of the IDS for Ergodic Operators. Let us now consider an ergodic family of self-adjoint operators $\left\{H_{\omega} \mid \omega \in \Omega\right\}$. With each operator we associate a finite-volume IDS $N_{\Lambda, \omega}^{X}(E)$. This is a random variable for each fixed $E$ and volume $\Lambda$. The ergodicity is essential as it introduces selfaveraging which implies the existence of a deterministic IDS for the family. We will show that the IDS exists using the superadditive ergodic theorem.

Let us consider the Dirichlet case $X=D$. First, we note that for fixed $E$, the family $N_{\Lambda, \omega}^{D}(E)$ is ergodic in the sense that

$$
N_{\Lambda, T_{x} \omega}^{D}(E)=N_{T_{x} \Lambda, \omega}^{D}(E),
$$

where $T_{x}$ is the translation by $x \in \mathbb{Z}^{d}$ or $x \in \mathbb{R}^{d}$. Second, suppose $\Lambda_{1}$ and $\Lambda_{2}$ are two regions with nonintersecting interiors and let $\Lambda \equiv \Lambda_{1} \cup \Lambda_{2}$. It follows from the Dirichlet boundary conditions that

$$
N_{\Lambda, \omega}^{D}(E) \geq N_{\Lambda_{1}, \omega}^{D}(E)+N_{\Lambda_{2}, \omega}^{D}(E) .
$$

This shows that the family of random variables $N_{\Lambda \omega}^{D}(E)$, indexed by $\Lambda$, is a superadditive ergodic process. Let $\Lambda_{n}$ denote the cubes $\Lambda_{n}(0)$. The Superadditive Ergodic Theorem states that if

$$
\sup _{n} \mathbb{E}\left(N_{\Lambda_{n}, \omega}^{D}(E)\right)<\infty
$$

then

$$
\lim _{n \rightarrow \infty} N_{\Lambda_{n}, \omega}^{D}(E)=\lim _{n \rightarrow \infty} \mathbb{E}\left(N_{\Lambda_{n}, \omega}^{D}(E)\right) \equiv N^{D}(E)
$$

exist $\mathbb{P}$-almost surely. For locally bounded potentials, the hypothesis (2.23) is easily verified. For more general potentials, the verification is given, for example, in [82]. As a consequence, the limit $N^{D}(E)$ is independent of the configuration.

For each energy $E$, there is a set $\Omega_{E}$ of $\mathbb{P}$-measure one for which the limit in (2.24) exists. The function $N^{D}(E)$ is a monotone increasing function. Let $S \subset \mathbb{R}$ be a countable set of points of continuity of $N^{D}(E)$. The set $\Omega_{0} \equiv \cap_{E \in S} \Omega_{E}$ is a set of $\mathbb{P}$-measure one. The monotonicity of $N^{D}(E)$ implies that for any point of continuity of $N^{D}(E)$, the limit on the left side of (2.24) exists for $\omega \in \Omega_{0}$. Hence, with probability one, the limit of the left side of (2.24) exists except for at most a countable number of points of discontinuity.

Since the limit function $N^{D}(E)$ is monotone increasing and continuous from the left, it is the distribution function of a measure $d N^{D}$ which is the DOS measure. 
We are often interested in proving more about the smoothness of the IDS which implies the absolutely continuity of the measure $d N^{D}$ with respect to Lebesgue measure. For example, if $N^{D}(E)$ is locally Lipschitz continuous, i. e. if the function $N^{D}(E)$ satisfies a condition such as

$$
\left|N^{D}(E)-N^{D}\left(E^{\prime}\right)\right| \leq C_{E}\left|E-E^{\prime}\right|,
$$

for some constant $C_{E}>0$, then the measure $d N^{D}$ is absolutely continuous with respect to Lebesgue measure. The Radon-Nikodym derivative of this measure is the actual density of states.

2.4.2. Pastur-Shubin Formula for the IDS. There is another expression for the DOS measure which is often convenient for calculations. Let $\chi_{\Lambda}$ be the characteristic function on the region $\Lambda$. We consider the mapping

$$
f \in C_{0}(\mathbb{R}) \rightarrow \operatorname{Tr}\left(\chi_{\Lambda} f(H) \chi_{\Lambda}\right),
$$

where $C_{0}(\mathbb{R})$ is the set of continuous functions of compact support on $\mathbb{R}$, and $\operatorname{Tr}$ denotes the trace on $L^{2}\left(\mathbb{R}^{d}\right)$. For a bounded region $\Lambda$, the operator $\chi_{\Lambda} f(H) \chi_{\Lambda}$ is trace class provided the potential $V$ is reasonable. To see this, let $g \in C_{0}^{\infty}(\mathbb{R})$ be another function such that $g \cdot f=f$. We can then write

$$
\begin{aligned}
\chi_{\Lambda} f(H) \chi_{\Lambda} & =\chi_{\Lambda} g(H) f(H) \chi_{\Lambda} \\
& =\left[\chi_{\Lambda}(1+H)^{-k}\right]\left[(1+H)^{k} g(H) f(H) \chi_{\Lambda},\right.
\end{aligned}
$$

for some $k>0$. Standard estimates show that for $k>d$, the operator $\chi_{\Lambda}(1+H)^{-k}$ is in the trace class. Consequently, we have the bound,

$$
\left|\operatorname{Tr}\left(\chi_{\Lambda} f(H) \chi_{\Lambda}\right)\right| \leq\left\|\chi_{\Lambda}(1+H)^{-k}\right\|_{1}\left\|(1+H)^{k} g(H)\right\|\|f\|_{\infty} .
$$

It follows from (2.28) that the mapping defined in (2.26) is continuous. The Riesz-Markov Theorem [110] states that there exists a positive Baire measure $\mu_{\Lambda}$ such that

$$
\operatorname{Tr}\left(\chi_{\Lambda} f(H) \chi_{\Lambda}\right)=\int f(E) d \mu_{\Lambda}(E) .
$$

This measure is the analog of the finite-volume density of states measure (2.14). However, it is defined with respect to the Hamiltonian $H$, rather than the finitevolume operator $H_{\Lambda}$. We can show, however, that the difference, being localized near the boundary of $\Lambda$ vanishes in the infinite-volume limit.

Lemma 2.15. Suppose that for $f \in C_{0}^{\infty}(\mathbb{R})$, we have

$$
\lim _{\Lambda \rightarrow \mathbb{R}^{d}}|\Lambda|^{-1} \operatorname{Tr}\left(\chi_{\Lambda} f(H) \chi_{\Lambda}\right) \equiv N(f)
$$

exists. Then, we have

$$
N(f)=\lim _{\Lambda \rightarrow \mathbb{R}^{d}}|\Lambda|^{-1} \operatorname{Tr}\left(f\left(H_{\Lambda}\right)\right) .
$$

Proof. It suffices to show that

$$
\lim _{\Lambda \rightarrow \mathbb{R}^{d}}|\Lambda|^{-1}\left|\operatorname{Tr}\left\{\chi_{\Lambda}\left(f(H)-f\left(H_{\Lambda}\right)\right) \chi_{\Lambda}\right\}\right|=0 .
$$

We use the Helffer-Sjöstrand formula (cf. [36]) to express $f(H)$ as an integral over the complex plane. Let $\tilde{f}$ be an almost analytic extension of $f$ vanishing to order $N$ 
as $y \rightarrow 0$. An almost analytic extension of $f$, that vanishing to order $N$ as $y \rightarrow 0$, can be constructed from the Taylor coefficients of $f$ by

$$
\tilde{f}(x+i y)=\sum_{k=0}^{N}(i y)^{k} \chi(y) \frac{f^{(k)}(x)}{k !},
$$

where $\chi$ is a smooth, nonnegative function of compact support equal to one in a neighborhood of zero. The formula for $f(H)$ is

$$
f(H)=\frac{1}{i \pi} \int_{G} d x d y \partial_{\bar{z}} \tilde{f}(z)(H-z)^{-1} .
$$

Let us assume that $\chi_{\Lambda}(H+1)^{-k}$ is in the trace class for $k$ suitably large. Replacing $f$ by $g(z)=(z+1)^{k} f(z)$, we can write

$$
\begin{gathered}
\chi_{\Lambda}\left(f(H)-f\left(H_{\Lambda}\right)\right) \chi_{\Lambda}= \\
\frac{1}{i \pi} \int_{a} d x d y \partial_{\bar{z}} \tilde{g}(z)\left\{\chi_{\Lambda}\left[(H+1)^{-k}(H-z)^{-1}-\left(H_{\Lambda}+1\right)^{-k}\left(H_{\Lambda}-z\right)^{-1}\right] \chi_{\Lambda}\right\} .
\end{gathered}
$$

We write the operator appearing in this expression as

$$
\begin{array}{r}
\chi_{\Lambda}(H+1)^{-k}(H-z)^{-1}\left\{\chi_{\Lambda}\left(H_{\Lambda}+1\right)^{k}\left(H_{\Lambda}-z\right)\right. \\
\left.-(H+1)^{k}(H-z)^{1} \chi_{\Lambda}\right\}\left(H_{\Lambda}+1\right)^{-k}\left(H_{\Lambda}-z\right)^{-1} \chi_{\Lambda} .
\end{array}
$$

Since $H_{\Lambda} \chi_{\Lambda}=H \chi_{\Lambda}$, we see that the quantity in the parentheses involves commutators of $H$ with $\chi_{\Lambda}$. Let us now choose $\chi_{\Lambda}$ to be of the form $\chi(x / R)$ where $\chi$ is smooth, has support in the ball of radius 2 , and is equal to one on the unit ball. The commutator of $\chi_{R}$ with $H$ is $O\left(R^{-1}\right)$. Hence, since the trace norms are $O\left(R^{d}\right)$, the extra decay from the commutator proves that the limit vanishes as $R \rightarrow \infty$.

2.4.3. Finite-Volume Operators. In the discussion of the DOS above, the finitevolume operators $H_{\Lambda}^{X}$ are defined with boundary conditions $X$ on $\partial \Lambda$. Finitevolume operators also play an important role in the multiscale analysis discussed in chapters 5 and 9. If we are concerned only with energies near the bottom of the the deterministic spectrum $\Sigma$, then the Dirichlet operators $H_{\Lambda}^{X}$ are suitable for the DOS and the multi-scale analysis. However, for energies near the band-edges of the deterministic spectrum $\Sigma$, another choice of the finite-volume operators is more appropriate. For example, we will study Schrödinger operators of the form $H_{\omega}=H_{0}+V_{\omega}$, where $H_{0}$ is a periodic Schrödinger operator with band spectrum. Under certain conditions, the deterministic spectrum of the random family will also have open spectral gaps near the gaps $G$ in the spectrum of $H_{0}$. In this case, we want to exploit the fact that the energies we are interested in lie in the unperturbed gap $G$ in the spectrum of $H_{0}$.

Let $V_{\Lambda} \equiv V_{\omega} \mid \Lambda$ be the local potential associated with $\Lambda$ and denote by $H_{\Lambda}$ the local Hamiltonian $H_{0}+V_{\Lambda}$. Note that since the local potential $V_{\Lambda}$ is a relativelycompact perturbation of $H_{0}$, the essential spectrum is unchanged. The effect of the local potential is to create at most finitely-many eigenvalues in the unperturbed gap $G$. We will also make use of the exponential decay of the resolvent of $H_{0}$ at energies in $G$. The same type of argument as in the proof of Lemma 2.15 shows that the DOS measure, and therefore the IDS, calculated from the local Hamiltonians $H_{\Lambda}=H_{0}+V_{\Lambda}$ agrees with the usual definition. 


\section{One-Parameter Families of Operators: Spectral Averaging and Perturbation of Singular Spectra}

3.1. Introduction. We begin with a discussion of a part of the theory which is of interest in its own right: one-parameter families of perturbations of a self-adjoint operator. Let $A$ be a self-adjoint operator and consider a self-adjoint perturbation $V$ that is relatively $A$-bounded with relative bound less than one. We will study one-parameter family of perturbations of $A$ by $V$ of the form $A(\lambda)=A+\lambda V$. The operators $A(\lambda)$ are self-adjoint on the domain $D(A)$ provided $|\lambda|<1$. As we will see, even the case when $V$ is a rank-one operator is of interest. The introduction of the parameter $\lambda$ allows us to work with averaged quantities. For example, if $\mu_{\lambda}$ is the spectral measure for $A(\lambda)$, we can study the average spectral measure $\int \mu_{\lambda} d \lambda$. Notice that this is a form of an expectation if we consider $\lambda$ as a random variable with a uniform, constant density. In certain cases, we will derive properties of the averaged measure. This will allow us to conclude that $\mu_{\lambda}$ has similar properties for almost every $\lambda$.

The random families of operators that we have considered are multiparameter perturbations of the background operator $H_{0}$. We reduce the problem to a one-parameter family in the following manner using the fact that the process is translation invariant. Let us consider a random family of Schrödinger operators $H_{\omega}=H_{0}+V_{\omega}$, where $V_{\omega}$ is an Anderson-type potential. We consider the set of configurations $\Omega_{0}$ for which all the coupling constants $\omega_{i}, i \neq 0$, are kept constant, but the coupling constant $\omega_{0}$ varies within the support of the density $g$. We can write the Hamiltonian for these configurations $\omega$ as

$$
H_{\omega}=H_{0}+V_{\omega^{\perp}}+\omega_{0} u,
$$

where $V_{\omega \perp}(x) \equiv \sum_{i \neq 0} \omega_{i} u(x-i)$ denotes the potential with the term at the zero site omitted. We now vary $\omega \rightarrow \widetilde{\omega}$ within the class of configurations $\Omega_{0}$ for which $\omega_{i}=\widetilde{\omega}_{i}$, for $i \neq 0$. We then have

$$
\begin{aligned}
H_{\widetilde{\omega}} & =H_{0}+V_{\omega}+\left(\widetilde{\omega}_{0}-\omega_{0}\right) u \\
& =H_{\omega}+\lambda u,
\end{aligned}
$$

where we defined a new parameter

$$
\lambda \equiv \widetilde{\omega}_{0}-\omega_{0} .
$$

Note that as operators, the Hamiltonian $H_{\widetilde{\omega}}$ and $H_{\omega}$ have exactly the same form and differ only in the coupling constant for the zero site. Hence, we can write equation (3.2) as

$$
H_{\lambda}=H+\lambda u,
$$

and study the effect of varying only one coupling constant. Of course, in the ergodic case, the choice of one site is equivalent to any other, so we can choose to study the effects of the variation of the coupling constant at the zero site without any loss of generality.

It was Simon and Wolff [119] who realized the importance of considering the variation of the coupling constant at one site in the proof of localization for random families of multidimensional Schrödinger operators on the lattice $\mathbb{Z}^{d}, d>1$. This allowed them to prove localization given the exponential decay estimates of Fröhlich and Spencer [56] on the resolvent. For the one dimensional case, Kotani [93] had also used the variation of a single boundary condition to obtain information about 
the spectral type of the deterministic spectrum. The classic studies of the effects on the spectrum of one-parameter families of (rank one) perturbations are due to Aronszajn [7] and Donoghue [39]. A nice review of the rank one theory and its application to random Schrödinger operators on the lattice is given in Simon's Vancouver lectures $[\mathbf{1 1 4}, \mathbf{1 1 5}]$.

In this chapter, we discuss extension of the results necessary for the proof of localization for multidimensional random operators on $\mathbb{R}^{d}$ for which the rank one theory is insufficient. We begin with spectral averaging results. Spectral averaging techniques play an important role in controlling the singular continuous spectrum of families of self-adjoint operators. Such methods have been used in the theory of random Schrödinger operators (cf. [21]), and in some approaches to quantum stability of time-dependent models [77]. We first present the simple spectral averaging method of Kotani and Simon [94], that they applied to the IDS, and used by Combes and the author [25] in the proof of localization. We will use this technique when we discuss the Wegner estimate and the Lipschitz continuity of the IDS in section 4. The method of Kotani and Simon [94] requires some analyticity of the potential in the random variables. We next present a technique applicable to more general families of potentials depending only differentiably on some parameter. The main tool is the method of differential inequalities. Finally, we present a refined spectral averaging result of [28] that is used to prove a Wegner estimate for general probability measures.

We then present a summary of the classical Aronszajn-Donoghue theory on rank-one perturbations. This is a beautiful theory and provides motivation for the second main result of this chapter on the perturbation of the singular spectrum. Our result presents an extension of this rank-one theory to a family of relatively compact perturbations arising in the theory. This allows us to eliminate the singular continuous spectrum for families of random operators.

A nice presentation of the classical Aronszajn-Donoghue theory and its applications to localization for random Schrödinger operators on the lattice is given by Simon in his Vancouver lectures, recently reprinted at the end of the reprinting of his trace ideals text $[\mathbf{1 1 4}]$.

3.2. Spectral Averaging. The basic idea behind spectral averaging is the following. Let $\mu_{\lambda}, \lambda \in \Gamma$, be a one-parameter family of real-valued measures on $\mathbb{R}$ with a common $\sigma$-algebra of measurable sets $\mathcal{B}$. Suppose that $g$ is a bounded, real-valued function on $\Gamma$. We can construct an averaged measure from the family in the following manner. For any subset $A \in \mathcal{B}$, define

$$
\nu(A) \equiv \int_{\Gamma} \mu_{\lambda}(A) g(\lambda) d \lambda
$$

The measure $\nu$ is obtained by averaging the measures $\mu_{\lambda}$ with respect to the weight function $g$. The main result of this section is that if the measures $\mu_{\lambda}$ are spectral measures of certain families of one-parameter self-adjoint operators, then the averaged measure $\nu$ is absolutely continuous with respect to Lebesgue measure. This result is often referred to as Kotani's trick in the literature about random Schrödinger operators.

3.2.1. The Kotani-Simon Spectral Averaging Method. The simplest spectral averaging result in the context of Schrödinger operators goes back to Kotani [93] who studied one-dimensional models. The result we present here appears in [94] and 
[25]. We consider the one-parameter family of operators $H_{\lambda}=H_{0}+\lambda V$, on a Hilbert space $\mathcal{H}$, where $H_{0}$ is self-adjoint and $V$ is a bounded operator satisfying

$$
0<c_{0} B^{2} \leq V,
$$

for some bounded self-adjoint operator $B$ and a finite, positive constant $c_{0}>0$.

TheOREM 3.1. Let $H_{\lambda}$ be as above and suppose that $V$ satisfies (3.6). For $\Im z<0$ and $\Im \lambda \geq 0$, we define the operator

$$
K(\lambda, z) \equiv B\left(H_{\lambda}-z\right)^{-1} B,
$$

and for $t>0$ and $\epsilon \geq 0$, we define

$$
F_{t}(\epsilon, z) \equiv \int_{\mathbb{R}} \frac{1}{1+t \lambda^{2}} K(\lambda+i \epsilon, z) d \lambda
$$

Then, for $c_{0}>0$ as in (3.6), we have

$$
\sup _{\epsilon>0}\left\|F_{t}(\epsilon, z)\right\| \leq \pi c_{0}^{-1} .
$$

Proof. Note that for $\Im z<0$ and $\Im \lambda=0$, the operator $K(\lambda, z)$ is bounded. We now establish an a priori bound on $K(\lambda, z)$. For $\Im z<0$ and $\Im \lambda \geq 0$, we easily verify that

$$
-\Im K(\lambda, z) \geq c_{0}(\Im \lambda) K(\lambda, z)^{*} K(\lambda, z)
$$

that implies

$$
\|K(\lambda, z)\| \leq \min \left(\left(c_{0} \Im \lambda\right)^{-1},|\Im z|^{-1}\|B\|^{2}\right) .
$$

We next note that the operator $K(\lambda, z)$ is analytic in $\lambda$ for $\Im \lambda>0$, and for fixed $z$, with $\Im z<0$. Furthermore, this operator is bounded in the upper-half complex $\lambda$-plane as in (3.11). Consequently, we can apply the Residue Theorem to evaluate the integral in the definition (3.8) and obtain

$$
F_{t}(\epsilon, z)=\pi t^{-1 / 2} K\left(i\left(t^{-1 / 2}+\epsilon\right), z\right) .
$$

The result (3.9) now follows from (3.12) and (3.11).

An immediate consequence of the bound (3.9) is the following estimate that we will use in chapter 4 . Suppose that $g \in L_{0}^{\infty}(\mathbb{R})$ is a nonnegative function. Then for any $\phi \in \mathcal{H}$, we have

$$
\left|\int_{\mathbb{R}} g(\lambda)\left\langle\phi,\left(H_{\lambda}-z\right)^{-1} \phi\right\rangle\right| \leq \pi c_{0}^{-1}\|g\|_{\infty}\|\phi\|^{2} .
$$

We obtain this from (3.9) by replacing the $g$ by $\left(1+t \lambda^{2}\right)^{-1}$ on the left in (3.13) at the cost of a factor $\sup _{\lambda}\left(g(\lambda)\left(1+t \lambda^{2}\right)\right)$, for any $t \geq 0$. Since the bound is independent of $t$, we can take $t=0$.

3.2.2. A Differential Inequality and Spectral Averaging. We now present a more local version of spectral averaging that does not require that the operator $H_{\lambda}$ depend linearly on $\lambda$ (or, more generally, analytically on $\lambda$ ). This is the case for the breather model (1.5.1). As in the proof in section 3.2.1, the proof of the spectral averaging theorem requires an a priori bound on the resolvent of the operators $H_{\lambda}$. We obtain this using a differential inequality method reminiscent of the method of Mourre [105] used to prove the limiting absorption principle for Schrödinger operators. 
TheOREM 3.2. Let $H_{\lambda}, \lambda \in \Gamma \equiv\left(\lambda_{0}, \lambda_{1}\right)$, be a $C^{2}$-family of self-adjoint operators on a separable Hilbert space $\mathcal{H}$ such that $D\left(H_{\lambda}\right)=D_{0} \subset \mathcal{H}, \forall \lambda \in \Gamma$, and such that $R_{\lambda}(z) \equiv\left(H_{\lambda}-z\right)^{-1}$ is twice strongly differentiable in $\lambda$ for all $z$, Im $z \neq 0$. Assume that there exist finite positive constants $C_{j}, j=0,1$, and bounded operator $B$, such that on $D_{0}$,

$$
\text { (D1) } \dot{H}_{\lambda} \equiv \frac{d H_{\lambda}}{d \lambda} \geq C_{0} B B^{*}>0 ;(D 2)\left|\ddot{H}_{\lambda}\right| \equiv\left|\frac{d^{2} H_{\lambda}}{d \lambda^{2}}\right| \leq C_{1} \dot{H}_{\lambda} .
$$

Then $\forall E \in \mathbb{R}$ and $\forall$ real, positive $g \in C_{0}^{2}(\Gamma)$, there exists a finite positive constant $C$, depending only on $\left\|g^{(j)}\right\|_{1}, j=0,1,2$, and the constants $C_{j}, j=0,1$ of (D1) and (D2), such that $\forall \phi \in \mathcal{H}$,

$$
\sup _{\delta>0}\left|\int_{\Gamma} g(\lambda)\left\langle\phi, B\left(H_{\lambda}-E-i \delta\right)^{-1} B \phi\right\rangle\right| \leq C\|\phi\|^{2} .
$$

\section{Remarks:}

(1) Although the proof of this abstract theorem requires some derivatives on the density $g$, there is a version in [25] that requires no derivatives on $g$.

(2) We will prove this theorem only for the linear case when $H_{\lambda}=H_{0}+\lambda u$. For this case, $\dot{H}_{\lambda}=u \geq 0$, and we can take $C_{0}=1$ and $B=u^{1 / 2}$ in (D1). Note that $\ddot{H}_{\lambda}=0$ so (D2) is automatically satisfied in this case. The proof is simpler if this holds because there is no error term to estimate.

\section{Proof of Theorem 3.2 in the Linear Case.}

The basic idea of the proof is to use differential inequality techniques (cf. [35, 105]).

1. a priori estimates. For $\epsilon>0$ and $0<\delta<1$, we define a modified resolvent by

$$
R(\lambda, \epsilon, \delta) \equiv\left(H_{\lambda}-E+i \delta+i \epsilon \dot{H}_{\lambda}\right)^{-1} .
$$

Since the operator $\dot{H}_{\lambda}$ is positive in the sense of (D1), we will use this to control the $\delta \rightarrow 0$ limit. We study the limiting behavior of the $B$-weighted resolvent

$$
K(\lambda, \epsilon, \delta)=B^{*} R(\lambda, \epsilon, \delta) B .
$$

To see that the operator $K$ is well-defined, we compute a bound depending on $\epsilon$ only. For $\phi \in \mathcal{H},\|\phi\|=1$, the Cauchy-Schwartz inequality gives

$$
\begin{aligned}
\|K(\lambda, \epsilon, \delta) \phi\| & \geq-\operatorname{Im}\langle\phi, K(\lambda, \epsilon, \delta) \phi\rangle \\
& \geq\left\langle\phi, B^{*} R^{*}(\lambda, \epsilon, \delta)\left(\delta+\epsilon \dot{H}_{\lambda}\right) R(\lambda, \epsilon, \delta) B \phi\right\rangle \\
& \geq C_{0} \epsilon\|K(\lambda, \epsilon, \delta) \phi\|^{2},
\end{aligned}
$$

where we used (D1) to replace $\dot{H}_{\lambda}$ by its lower bound $C_{0} B B^{*}$. Hence, we obtain

$$
\|K(\lambda, \epsilon, \delta)\| \leq\left(C_{0} \epsilon\right)^{-1} .
$$

We also need a related result for $K(\lambda, \epsilon, \delta)^{*}$. Note that

$$
K(\lambda, \epsilon, \delta)^{*}=K(\lambda,-\epsilon,-\delta),
$$

so that in place of (3.12), we have

$$
\begin{aligned}
\left\|K(\lambda, \epsilon, \delta)^{*} \phi\right\| & \geq \operatorname{Im}\langle\phi, K(\lambda,-\epsilon,-\delta) \phi\rangle \\
& \geq \epsilon C_{0}\left\|K(\lambda, \epsilon, \delta)^{*} \phi\right\|^{2} .
\end{aligned}
$$


2. First differential inequality. Let $g \in C_{0}^{2}(\Gamma)$, and define

$$
F(\epsilon, \delta) \equiv \int_{\Gamma} g(\lambda)\langle\phi, K(\lambda, \epsilon, \delta) \phi\rangle d \lambda
$$

By (3.13), we obtain the bound

$$
|F(\epsilon, \delta)| \leq(C \epsilon)^{-1}\|g\|_{1} .
$$

We first compute the $\epsilon$-derivative of $F$,

$$
i \frac{d F}{d \epsilon}(\epsilon, \delta)=\int_{\Gamma} g(\lambda)\left\langle\phi, B R(\lambda, \epsilon, \delta) \dot{H}_{\lambda} R(\lambda, \epsilon, \delta) B \phi\right\rangle d \lambda .
$$

On the other hand, we note that

$$
\frac{d}{d \lambda}\langle\phi, K(\lambda, \epsilon, \delta) \phi\rangle=-\left\langle B \phi, R \dot{H}_{\lambda} R B \phi\right\rangle,
$$

using the fact that $\ddot{H}_{\lambda}=0$ in this case. We substitute this into the right side of (3.17) to obtain

$$
i \frac{d F}{d \epsilon}(\epsilon, \delta)=-\int_{\Gamma} g(\lambda) \frac{d}{d \lambda}\langle\phi, K(\lambda, \epsilon, \delta) \phi\rangle .
$$

We integrate by parts with respect to $\lambda$ in the first term on the right to obtain

$$
i \frac{d F}{d \epsilon}(\epsilon, \delta)=\int_{\Gamma} g^{\prime}(\lambda)\langle\phi, K(\lambda, \epsilon, \delta) \phi) .
$$

Using the a priori estimates, we obtain

$$
\begin{aligned}
\left|\frac{d F}{d \epsilon}(\epsilon, \delta)\right| & \leq \int_{\Gamma} g^{\prime}(\lambda)\langle\phi, K(\lambda, \epsilon, \delta) \phi\rangle \mid \\
& \leq\left(\epsilon C_{0}\right)^{-1}\left\|g^{\prime}\right\|_{1} .
\end{aligned}
$$

Integrating this differential inequality yields an improved estimate for $F$,

$$
|F(\epsilon, \delta)| \leq C_{3}|\log \epsilon|+|F(1, \delta)|,
$$

where $C_{3}$ is independent of $\delta$ and $|F(1, \delta)|$ is uniformly bounded as $\delta \downarrow 0$.

3. Iteration. With the new bound (3.27) for $F(\epsilon, \delta)$, we repeat the above procedure in order to obtain an improved upper bound for $F(\epsilon, \delta)$ which remains finite as $\epsilon \rightarrow 0$. To do this, we need a better estimate on

$$
\int_{\Gamma} g^{\prime}(\lambda)\langle\phi, K \phi\rangle d \lambda
$$

We define another function $\tilde{F}(\epsilon, \delta)$ by

$$
\tilde{F}(\epsilon, \delta)=\int g^{\prime}(\lambda)\langle\phi, K(\lambda, \epsilon, \delta) \phi\rangle d \lambda .
$$

As in (3.16), $\tilde{F}$ satisfies

$$
|\tilde{F}(\epsilon, \delta)| \leq\left(C_{0} \epsilon\right)^{-1}|| g^{\prime} \|_{1} .
$$

We now repeat the arguments of part 2 of the proof for this function $\tilde{F}$. We obtain an estimate similar to (3.26),

$$
\left|\frac{d \tilde{F}}{d \epsilon}(\epsilon, \delta)\right| \leq\left(\epsilon C_{0}\right)^{-1} C_{1}\left\|g^{\prime}\right\|_{1} .
$$


Integration of this inequality leads to,

$$
|\tilde{F}(\epsilon, \delta)| \leq C_{4}|\log \epsilon|+|\tilde{F}(1, \delta)| .
$$

4. Conclusion. With this new estimate (3.32), we return to (3.27) and obtain

$$
\left|\frac{d F}{d \epsilon}(\epsilon, \delta)\right| \leq C_{5}|\log \epsilon|+C_{6},
$$

where $C_{5}$ is independent of $\delta$ and depends on $\left\|g^{(p)}\right\|_{1}, p=0,1$ and $C_{6}$ depends on $|F(1, \delta)|$ and $|\tilde{F}(1, \delta)|$, which are bounded, independent of $\delta$. We now integrate the inequality (3.33) and obtain,

$$
|F(\epsilon, \delta)| \leq C,
$$

where $C$ depends on $\left\|g^{(p)}\right\|_{1}, p=0,1$ and is independent of $\delta$ and uniform in $\epsilon, 0<$ $\epsilon<1$. The proof of the theorem now follows from the fact that $R(\lambda, \epsilon, \delta)$ converges weakly to $R(\lambda, \delta)$, as $\epsilon \rightarrow 0$, provided $\delta>0$, and the dominated convergence theorem since

$$
\left|\int_{\Gamma} g(\lambda)\langle\phi, B R(\lambda, \epsilon, \delta) B \phi\rangle d \lambda\right| \leq C
$$

by $(3.34)$.

3.2.3. A General Spectral Averaging Method. If we want to treat more general probability measures, like Hölder continuous measures, we need a more refined and local version of spectral averaging in order to prove a Wegner estimate, as discussed in chapter 4 . Let us consider a probability measure $\mu$ and define the Levy concentration of the measure, for any $\epsilon>0$, by

$$
s(\epsilon) \equiv \sup _{E \in \mathbb{R}} \mu([E, E+\epsilon]) .
$$

If $\mu$ is Hölder continuous with exponent $0<\alpha \leq 1$, then $s(\epsilon) \sim \epsilon^{\alpha}$. Spectral averaging with respect to such general probability measures relies on the following basic result of $[\mathbf{2 8}]$ that is a discrete version of spectral averaging.

Proposition 3.1. Let $A$ and $B$ be two self-adjoint operators on a separable Hilbert space $\mathcal{H}$, and suppose that $B \geq 0$ is bounded. Then, for any $\phi \in \mathcal{H}$, we have the bound

$$
\sum_{n \in \mathbb{Z}} \sup _{y \in[0,1]}\left\langle B \phi, \frac{1}{(A+(n+y) B)^{2}+1} B \phi\right\rangle \leq \pi\left(\|B\|+\|B\|^{2}\right)\|\phi\|^{2} .
$$

We do not prove this abstract result here but refer to [28], mentioning that the proof uses results from the theory of maximally dissipative operators. The application of this result to random Schrödinger operators is given in the following proposition.

THEOREM 3.3. Let $H_{\lambda}=H_{0}+\lambda V$ be self-adjoint on a separable Hilbert space $\mathcal{H}$ with $V \geq 0$ bounded and suppose that $\lambda$ is distributed according to a probability measure $\mu$ with Levy concentration $s(\epsilon)$, defined in (3.35). For any $\epsilon>0$, let $\Delta_{\epsilon} \subset \mathbb{R}$ be an interval with $\left|\Delta_{\epsilon}\right|=\epsilon$. We have the following bound on the average of the matrix element of the imaginary part of the resolvent:

$$
\int_{\Delta_{\epsilon}} d E \int_{\mathbb{R}} d \mu(\lambda) \Im\left\langle\phi, V\left(\frac{1}{H_{\lambda}-E-i \epsilon}\right) V \phi\right\rangle \leq \pi\|V\|(1+\|V\|) s(\epsilon)\|\phi\|^{2} .
$$


Proof: The imaginary part of the matrix element in (3.37) is

$$
\left\langle V \phi, \frac{\epsilon}{\left(H_{0}-E+\lambda V\right)^{2}+\epsilon^{2}} V \phi\right\rangle=\frac{1}{\epsilon}\left\langle V \phi, \frac{1}{\epsilon^{-2}\left(H_{0}-E+\lambda V\right)^{2}+1} V \phi\right\rangle .
$$

To apply Proposition 3.1, we choose $B=V$ and define a self-adjoint operator $A \equiv \epsilon^{-1}\left(H_{0}-E\right)$ so the matrix element in (3.38) may be written as

$$
\left\langle V \phi, \frac{1}{\left(A+\epsilon^{-1} \lambda V\right)^{2}+1} V \phi\right\rangle .
$$

We divide the integration over $\lambda$ into a sum over intervals $[n \epsilon,(n+1) \epsilon]$, and change variables letting $\lambda / \epsilon=n+y$, so that $y \in[0,1]$. We then obtain

$$
\begin{array}{rl}
\int_{\mathbb{R}} & d \mu(\lambda)\left\langle V \phi, \frac{1}{\left(A+\epsilon^{-1} \lambda V\right)^{2}+1} V \phi\right\rangle \\
& =\sum_{n} \int_{n \epsilon}^{(n+1) \epsilon} d \mu(\lambda)\left\langle V \phi, \frac{1}{(A+(n+y) V)^{2}+1} V \phi\right\rangle \\
& \leq\left(\sup _{m \in \mathbb{Z}} \mu([m \epsilon,(m+1) \epsilon])\right)\left\{\sum_{n} \sup _{y \in[0,1]}\left\langle V \phi, \frac{1}{(A+(n+y) V)^{2}+1} V \phi\right\rangle\right\} .
\end{array}
$$

We apply Proposition 3.1 to the last line in (3.40) and obtain

$$
\int_{\mathbb{R}} d \mu(\lambda)\left\langle V \phi, \frac{1}{\left(A+\epsilon^{-1} \lambda V\right)^{2}+1} V \phi\right\rangle \leq \pi\|V\|(1+\|V\|) s(\epsilon)\|\phi\|^{2}
$$

This provides a bound for the average over $\lambda$ of (3.38). Integrating in energy over $\Delta_{\epsilon}$, and recalling the factor of $\epsilon^{-1}$ in (3.38), we obtain the estimate (3.37).

We note that when $\mu$ has a density $g \in L_{0}^{\infty}(\mathbb{R})$, we recover the estimate in Theorem 3.1.

3.2.4. A Result on Averaged Spectral Projections. An immediate application of all three spectral averaging theorems, Theorem 3.1, Theorem 3.2, or Theorem 3.3, is the following result on the averaged spectral projectors of the Hamiltonian $H_{\lambda}$. Let $E_{\lambda}(A)$ be the spectral projection for the self-adjoint operator $H_{\lambda}$ and the subset $A \subset \mathbb{R}$.

COROLlary 3.1. Let $H_{\lambda}$ be a one-parameter family of self-adjoint operators as in Theorem 3.1, Theorem 3.2, or Theorem 3.3. Let $\lambda$ be distributed with a density $0 \leq g \in L_{0}^{\infty}(\mathbb{R})$, or $g \in C_{0}^{2}(\Gamma)$, or a general probability measure $\mu$, respectively. Then, for the cases of Theorems 3.1 and 3.2, we have that for any Borel set $J \subset \mathbb{R}$, there exists a finite positive constant $C_{g}>0$, depending only on $\|g\|_{\infty}$ for Theorem 3.1 , or on $\left\|g^{(j)}\right\|_{1}, j=0,1,2$ for Theorem 3.2, such that

$$
\left\|\int_{\Gamma} g(\lambda) B^{*} E_{\lambda}(J) B\right\| \leq C_{g}|J| .
$$

As a consequence, for any $\phi \in \mathcal{H}$, with $\|\phi\|=1$, we have

$$
\int_{\Gamma} g(\lambda)\left\langle B \phi, E_{\lambda}(J) B \phi\right\rangle \leq C_{g}|J| .
$$

For the general case of Theorem 3.3, the analogues of (3.42) and (3.43) hold with $|J|$ replaced by $s(|J|)$, for $|J|$ sufficiently small. 
Proof. One uses Stone's formula for spectral projections to write

$$
\left\langle\phi, B E_{\lambda}(J) B \phi\right\rangle \leq \frac{1}{\pi} \lim _{\delta \downarrow 0} \operatorname{Im} \int_{J} d E\left\langle\phi, B^{*}\left(H_{\lambda}-E-i \delta\right)^{-1} B \phi\right\rangle .
$$

It is easy to show that

$$
B^{*}\left(H_{\lambda}-E-i \delta\right)^{-1} B=n-\lim _{\epsilon \downarrow 0} K(\lambda, \epsilon, \delta) .
$$

Substituting $K$ into the right side of (3.2.4), the result follows from any of the spectral averaging Theorems 3.1, 3.2, or 3.3.

3.2.5. Kotani's Trick. This technique is based on the theorem that the averaged spectral measure is absolutely continuous with respect to Lebesgue measure. Let $E_{\lambda}(\cdot)$ be the spectral family for $H_{\lambda}$.

Corollary 3.2. In addition to the hypotheses of Theorem 3.2, assume that Ran $B$ is cyclic $\forall H_{\lambda}, \lambda \in \Gamma$, in the sense that $\left\{f\left(H_{\lambda}\right) B \phi \mid f \in L^{\infty}(\mathbb{R}), \phi \in \mathcal{H}\right\}$ is dense in $\mathcal{H}$. Then for any Borel set $J \subset \mathbb{R}$ with $|J|=0$, one has $E_{\lambda}(J)=0$ almost every $\lambda \in \Gamma$.

Proof. Corollary 3.1 shows that

$$
\int_{\Gamma} h(\lambda)\left\langle\phi, B^{*} E_{\lambda}(J) B \phi\right\rangle d \lambda=0,
$$

implies that

$$
\left\langle\phi, B^{*} E_{\lambda}(J) B \phi\right\rangle=0,
$$

for almost every $\lambda \in \Gamma$. This set of $\lambda$, however, depends on $\phi$, and we denote it by $\Gamma_{\phi}$. The $H$-cyclicity assumption on $B$ implies that for each $\psi$ in a dense set there exists a set $\Gamma_{\psi}$ such that $\left\langle\psi, E_{\lambda}(J) \psi\right\rangle=0$, for all $\lambda \in \Gamma_{\psi}$, and $\left|\Gamma_{\psi}\right|=|\Gamma|$. Now let $\left\{\psi_{n}\right\}$ be a complete orthonormal basis for $\mathcal{H}$ and set $\Gamma_{\infty} \equiv \bigcap_{n} \Gamma_{\psi_{n}}$. Then $\left|\Gamma_{\infty}\right|=|\Gamma|$ and $\left\langle\psi, E_{\lambda}(J) \psi\right\rangle=0, \forall \lambda \in \Gamma_{\infty}$ and $\forall \psi$ in a dense set in $\mathcal{H}$. By standard arguments, this can be extended to all $\psi \in \mathcal{H}$. Since $E_{\lambda}$ is a projection, this shows $E_{\lambda}(J)=0$ a.e. $\lambda \in \Gamma$.

3.3. Review of Aronszajn-Donoghue Theory. The theory of one-parameter families presented section 3.2 is inspired by the theory of rank one perturbations developed by Aronszajn [7] and Donoghue [39]. We review this theory here because of its interest and transparency. A comprehensive discussion of recent developments in the theory of rank-one perturbations is given by Simon in [115]. Let $\mu$ be a real, nonnegative measure on $\mathbb{R}$ satisfying

$$
\int \frac{d \mu(\lambda)}{1+|\lambda|}
$$

Definition 3.4. The Borel transform $F_{\mu}(z)$ of a real, nonnegative measure on $\mathbb{R}$, satisfying (3.48), for $z=E+i \epsilon \in \mathbb{C}$ with $\operatorname{Im} z=\epsilon \neq 0$, is defined by

$$
F_{\mu}(z) \equiv \int \frac{d \mu(\lambda)}{\lambda-z} .
$$

It is useful to record here the real and imaginary parts of the Borel transform, both of which exist provided $\epsilon \neq 0$ :

$$
\operatorname{Im} F_{\mu}(E+i \epsilon)=\epsilon \int \frac{d \mu(\lambda)}{(\lambda-E)^{2}+\epsilon^{2}}
$$


and

$$
\operatorname{Re} F_{\mu}(E+i \epsilon)=\int \frac{(\lambda-E) \mu(\lambda)}{(\lambda-E)^{2}+\epsilon^{2}} .
$$

In preparation for the Aronszajn-Donoghue Theorem, our first goal is to relate the Lebesgue decomposition of a measure $\mu$ into its singular $\mu_{s}$ and absolutely continuous $\mu_{a c}$ parts to the behavior of the boundary-value of the Borel transform of the measure $F_{\mu}$. In the applications of interest to us, the measure $\mu$ will be a spectral measure of a self-adjoint operator. We will see that the Borel transform is related to matrix elements of the resolvent of that self-adjoint operator.

We recall that a measure $\nu$ is supported on a subset $S \subset \mathbb{R}$ if $\nu(\mathbb{R} \backslash S)=0$. We will also need the function $B_{\mu}(E+i \epsilon)$ defined by

$$
B(E+i \epsilon) \equiv\left[\int \frac{d \mu(\lambda)}{(E-\lambda)^{2}+\epsilon^{2}}\right]^{-1} .
$$

Proposition 3.2. Let $\mu$ be a real, nonnegative measure on $\mathbb{R}$ satisfying (3.48). We define the following subsets of $\mathbb{R}$ :

$$
\begin{array}{r}
S_{\mu} \equiv\left\{E \mid \lim _{\epsilon \rightarrow 0} \operatorname{Im} F_{\mu}(E+i \epsilon)=\infty\right\} \\
C_{\mu} \equiv\left\{E \mid \lim _{\epsilon \rightarrow 0} \operatorname{Im} F_{\mu}(E+i \epsilon)>0 \text { and finite }\right\} \\
P_{\mu} \equiv\left\{E \mid E \in S_{\mu}, B_{\mu}(E)=0, \text { and } \lim _{\epsilon \rightarrow 0} \epsilon \operatorname{Im} F_{\mu}(E+i \epsilon) \neq 0\right\} .
\end{array}
$$

Then, $\mu$ is supported on $S_{\mu} \cup C_{\mu}$, the measure $\mu \mid S_{\mu}$ is singular with respect to Lebesgue measure, the measure $\mu \mid C_{\mu}$ is absolutely continuous with respect to Lebesgue measure, and $\mu \mid P_{\mu}$ is a pure point measure. Furthermore, the density of $\mu \mid C_{\mu}$ is given by

$$
\frac{d \mu_{a c}}{d E}(E)=\lim _{\epsilon \rightarrow 0} \frac{1}{\pi} \operatorname{Im} F_{\mu}(E+i \epsilon)
$$

and for any $E_{0}$,

$$
\mu\left(\left\{E_{0}\right\}\right)=\lim _{\epsilon \rightarrow 0} \epsilon \operatorname{Im} F_{\mu}(E+i \epsilon) .
$$

\section{Sketch of the Proof.}

1. For any continuous function $f$ of compact support, it is easy to show that

$$
\lim _{\epsilon \rightarrow 0} \int f(E) \frac{1}{\pi} \operatorname{Im} F_{\mu}(E+i \epsilon) d E=\int f(E) d \mu(E) .
$$

Consequently, the weak limit as $\epsilon \rightarrow 0$ of $\operatorname{Im} F_{\mu}(E+i \epsilon)$ exists for Lebesgue almostevery $E \in \mathbb{R}$. Furthermore, it is a classical result, called Fatou's Lemma, that the pointwise $\lim _{\epsilon \rightarrow 0} \operatorname{Im} F_{\mu}(E+i \epsilon)$ exists almost everywhere. The limit is exactly the Radon-Nikodym derivative $\frac{d \mu_{a c}}{d E}(E)$ of $\mu$ with respect to Lebesgue measure. The theorem of de la Vallée Poussin states that the singular part of the measure is supported on the set $S_{\mu}$.

2. An application of the Lebesgue Dominated Convergence Theorem shows that

$$
\mu\left(\left\{E_{0}\right\}\right)=\lim _{\epsilon \rightarrow 0} \epsilon \operatorname{Im} F_{\mu}\left(E_{0}+i \epsilon\right) .
$$

If this is nonzero, it follows that $E_{0} \in S_{\mu}$. Now suppose the measure $\mu$ has an atom at $E_{0}$ with weight $C\left(E_{0}\right)$. Then the function $B_{\mu}(E)$ satisfies

$$
\lim _{\epsilon \rightarrow 0} \epsilon B_{\mu}\left(E_{0}+i \epsilon\right)^{-1}=C\left(E_{0}\right) .
$$


Hence, the set $P_{\mu}$ supports the pure point part of the measure $\mu$.

We mention a related criterion for absolutely continuity of a measure which was recently proved and used by Klein $[\mathbf{8 8}]$ in the study of extended states for the Anderson model on the Bethe lattice.

Proposition 3.3. Let $\mu$ be a real, nonnegative, measure $\mu$ on $\mathbb{R}$ satisfying (3.48) with Borel transform $F_{\mu}(z)$. If

$$
\lim _{\epsilon \rightarrow 0^{+}} \int_{a}^{b}\left|F_{\mu}(E+i \epsilon)\right|^{2} d E<\infty,
$$

then $\mu \mid(a, b)$ is absolutely continuous with respect to Lebesgue measure.

Another proof of this result, and related results concerning the relation between properties of the measure and the boundary behavior of the $L^{p}$-norm of the Borel transform, are given in Simon [117].

The subject of the Aronszajn-Donoghue theory is the instability of the singular spectrum under rank-one perturbations. It is well-known (cf. [81]) that the absolutely continuous spectrum is stable under a trace class perturbation. Let $H_{0}$ be a self-adjoint operator on a separable Hilbert space $\mathcal{H}$. We assume that $H_{0}$ has a cyclic vector $\phi \in \mathcal{H}$ and form the rank-one, orthogonal projector $P \equiv|\phi\rangle\langle\phi|$. We consider the one-parameter family of operators

$$
H_{\lambda} \equiv H_{0}+\lambda P \text {. }
$$

The resolvent of this family is denoted $R_{\lambda}(z)=\left(H_{\lambda}-z\right)^{-1}$. Since $\phi$ is cyclic for $H_{0}$, the spectral measure $\mu_{0, \phi}$, defined by

$$
\left\langle\phi, R_{0}(z) \phi\right\rangle=\int \frac{d \mu_{0, \phi}(\eta)}{\eta-z},
$$

determines the operator $H_{0}$. Similarly, the measure $\mu_{\lambda, \phi}$ determines $H_{\lambda}$ since $\phi$ is also cyclic for each $H_{\lambda}$.

We have seen that information about a measure can be recovered from the boundary-value of its Borel transform. We want to obtain information about the perturbed measure $\mu_{\lambda, \phi}$, and hence about the perturbed operator $H_{\lambda}$, from knowledge of the boundary behavior of the Borel transform of the measure $\mu_{0, \phi}$, corresponding to $H_{0}$. In the rank-one perturbation case, the Borel transform of the spectral measure is just the matrix element of the resolvent. Hence, we define

$$
F_{\lambda}(z) \equiv\left\langle\phi, R_{\lambda}(z) \phi\right\rangle .
$$

A key role is played by the equation relating $F_{\lambda}(z)$ to $F_{0}(z)$. To derive this equation, we begin with the second resolvent formula,

$$
R_{\lambda}(z)=R_{0}(z)-\lambda R_{\lambda}(z) P R_{0}(z) .
$$

Upon taking the matrix element of this equation in the state $\phi$, we find

$$
F_{\lambda}(z)\left(1+\lambda F_{0}(z)\right)=F_{0}(z) .
$$

When the factor $\left(1+\lambda F_{0}(z)\right)$ is invertible, we can deduce the behavior of $F_{\lambda}(E+i \epsilon)$, as $\epsilon \rightarrow 0$, from the behavior of $F_{0}(E+i \epsilon)$ in the same limit. Note that the beauty of a rank one perturbation is that this equation (3.22) is simply a relation between functions which are analytic in the upper half-plane.

The first main result of the Aronszajn-Donoghue analysis is the following theorem. 
TheOrem 3.5. Relative to the function $F_{0}(E+i \epsilon)$ defined above, we define the following sets for $\lambda \neq 0$,

$$
\begin{gathered}
C \equiv\left\{E \mid \lim _{\epsilon \rightarrow 0} \operatorname{Im} F_{0}(E+i \epsilon)=\Phi(E)>0 \text { and finite }\right\} \\
S_{\lambda} \equiv\left\{E \mid \lim _{\epsilon \rightarrow 0} F_{0}(E+i \epsilon)=-1 / \lambda\right\}
\end{gathered}
$$

Then, the set $C$ is the support of $\mu_{\lambda, a c}$, for all $\lambda$, and the set $S_{\lambda}$ is the support of $\mu_{\lambda, s}$, for $\lambda \neq 0$.

Of course, the absolutely continuous spectrum is invariant under finite-rank perturbations, and this fact is reflected by the first part of the theorem. The singular spectrum, however, is very unstable with respect to the perturbation. One of the most interesting consequences of Theorem 3.3 concerns the singular spectrum of $H_{\lambda}$.

COROLlary 3.3. Under the conditions of Theorem 3.5,

(1) The absolutely continuous parts of $H_{\lambda}$ and $H_{\beta}$ are unitarily equivalent;

(2) The singular components of the spectral measures of $H_{\lambda}$ and $H_{\beta}$, for $\lambda \neq \beta$, are mutually singular.

We recall that the second part of the corollary means that the if $\mu_{\lambda_{i}, s}$ are the singular parts of the spectral measures for $H_{\lambda_{1}}$ and $H_{\lambda_{2}}$, for $0 \neq \lambda_{1} \neq \lambda_{2} \neq 0$, then they are mutually singular if $\mu_{\lambda_{i}, s}\left(S_{\lambda_{j}}\right)=0$, for $i \neq j$, where $S_{\lambda_{j}}$ is the set defined in (3.68).

With regard to the eigenvalues of $H_{\lambda}$, for $\lambda \neq 0$, we have the following finer decomposition of the singular set $S_{\lambda}$ in (3.68).

COROLlary 3.4. As in framework of Theorem 3.5, we define the function $B_{0}(E)$ by

$$
B_{0}(E) \equiv\left[\int_{I} R \frac{d \mu_{0}(s)}{(s-E)^{2}}\right]^{-1} .
$$

Suppose that $E$ is not an eigenvalue of $H_{0}$. Then, the energy $E$ is an eigenvalue of $H_{\lambda}$ if and only if $E \in S_{\lambda}$, where $S_{\lambda}$ is defined in (3.68), and $B_{0}(E)>0$. Consequently, $E \in \sigma_{s c}\left(H_{\lambda}\right)$ if and only if $E \in S_{\lambda}$ and $B_{0}(E)=0$.

3.4. Perturbation of Singular Spectra. Our goal is to extend the ideas of the Aronszajn-Donoghue theory to relatively compact perturbations. This is essential for the our treatment of multidimensional, continuous random operators. A similar analysis for Schrödinger operators was performed by Howland [76].

Analogous to (3.2)-(3.4), we consider one parameter families of the form

$$
H_{\lambda}=H_{0}+\lambda V, \lambda \in \mathbb{R} .
$$

We require that $V$ is a nonzero, bounded, self-adjoint operator and that it admits a factorization of the form $V=C D^{*}$. In the applications, $V=u$, the singlesite potential, so we can take $C=D=u^{1 / 2}$. As in section 3.2 , we set $R_{\lambda}(z)=$ $\left(H_{\lambda}-z\right)^{-1}$, for $\lambda \in \mathbb{R}$. We consider a fixed energy interval $I \subset \mathbb{R}$. We need the 
following assumptions

$$
\Gamma(z) \equiv D^{*} R_{0}(z) C \text { is compact } \forall z, \operatorname{Im} z \neq 0 ;
$$

(A2) there exists a subset $I_{0} \subset I,\left|I_{0}\right|=|I|$, and constants $C(E)<\infty$, such that

$$
\begin{aligned}
& \sup _{\epsilon \neq 0}\left\|R_{0}(E+i \epsilon) X\right\| \leq C(E)<\infty \\
& \forall E \in I_{0} \text { and } X=C \text { and } D .
\end{aligned}
$$

We show that (A2) is a sufficient condition for the absence of singular continuous spectrum of $H_{\lambda}$ in $I$ for almost-every $\lambda$ in the case that $C= \pm D$. This is a variation of the instability of singular spectrum result presented in Theorem 3.5. As above, let $\tilde{\mathcal{H}}$ be the cyclic subspace of $\mathcal{H}$ generated by $C$ and $H_{\lambda}$ :

$$
\tilde{\mathcal{H}}=\left[f\left(H_{\lambda}\right) C \phi, f \in L^{\infty}(\mathbb{R}), \phi \in \mathcal{H}\right]^{\text {closure }} .
$$

We write $\tilde{H}_{\lambda}$ for the restriction of $H_{\lambda}$ to the invariant subspace $\tilde{\mathcal{H}}$, which is independent of $\lambda$.

THEOREM 3.6. Let us assume that the operator $H_{0}$ and the perturbation $V=$ $C D^{*}$ satisfy (A1) and (A2). We then have

$$
\begin{aligned}
& \text { (1) } \sigma_{a c}\left(\tilde{H}_{\lambda}\right) \cap I=\emptyset, \forall \lambda \in \mathbb{R} ; \\
& \text { (2) } \sigma\left(\tilde{H}_{\lambda}\right) \cap I_{0} \text { is pure point, } \forall \lambda \in \mathbb{R} \backslash\{0\} .
\end{aligned}
$$

If, in addition, $C= \pm D$, then we have

$$
\text { (3) } \sigma_{s c}\left(\tilde{H}_{\lambda}\right) \cap I=\emptyset \text { for Lebesgue almost every } \lambda \in \mathbb{R} \text {. }
$$

Consequently, the spectrum $\tilde{H}_{\lambda}$, for Lebesgue almost-every $\lambda \in \mathbb{R}$, is pure point in $I$ with finitely-degenerate eigenvalues.

Proof. 1. We omit the tildes for simplicity of notation. From the second resolvent equation, we can express $R_{\lambda}(z)$ in terms of $R_{0}(z)$ and $\Gamma(z) \equiv D^{*} R_{0}(z) C$. We obtain

$$
D^{*} R_{\lambda}(z) C(1+\lambda \Gamma(z))=\Gamma(z)
$$

which is the analogue of the Aronszajn-Donoghue formula (3.66) in our case. In accordance with the Aronszajn-Donoghue theory, we want to study the boundary values of $R_{\lambda}(E+i \epsilon)$, as $\epsilon \rightarrow 0^{+}$, given the information about the boundary values of $R_{0}(E+i \epsilon)$ given in (A2). We first note that for all $\lambda \in \mathbb{R}$, the operator $(1+\lambda \Gamma(z))$ is invertible for $z \in \mathbb{C}$ with $\operatorname{Im} z \neq 0$. If it were not invertible for some $\lambda$ and $z \in \mathbb{C}$, with $\operatorname{Im} z \neq 0$, then condition (A1) and the Fredholm alternative for compact operators implies that $\exists \psi \in \mathcal{H}$ such that

$$
(1+\lambda \Gamma(z)) \psi=0 .
$$

This equation is equivalent to

$$
\lambda D^{*} \frac{1}{H_{0}-z} C \psi=-\psi .
$$

Multiplying both sides by $C$ and defining $\xi=\left(H_{0}-z\right)^{-1} C \psi \in \mathcal{H}$, we get

$$
-\lambda V \xi=\left(H_{0}-z\right) \xi
$$


or,

$$
\left(H_{\lambda}-z\right) \xi=0
$$

which shows $H_{\lambda}$ has a complex eigenvalue, which is impossible since $H_{\lambda}$ is selfadjoint.

2. We next study the behavior of the boundary-values of $\Gamma(E+i \epsilon)$. For $E \in I_{0}$, condition (A2) implies that $n-\lim _{\epsilon \rightarrow 0} \Gamma(E+i \epsilon) \equiv \Gamma(E+i 0)$ exists. This operator is also compact by (A1). It now follows by the Bounded Inverse Theorem and the Fredholm alternative that for $E \in I_{0}$,

$$
n-\lim _{\epsilon \rightarrow 0^{+}}(1+\lambda \Gamma(E+i \epsilon))^{-1}=(1+\lambda \Gamma(E+i 0))^{-1}
$$

exists if and only if there is no $\eta \in \mathcal{H}$ such that

$$
\left(1+\lambda \Gamma\left(E+i 0^{+}\right)\right) \eta=0 .
$$

We will show that this equation holds only at energies $E \in I_{0}$ that are eigenvalues of $H_{\lambda}$. The idea is as in (1) but now one has to control the limit of $\eta_{\epsilon} \equiv R_{0}(E+i \epsilon) C \eta$. Since condition (A2) implies that $\left\|\eta_{\epsilon}\right\| \leq C(E)$, we have that $\eta_{0} \equiv w-\lim _{\epsilon \rightarrow 0} \eta_{\epsilon}$ exists. We now need to show that $\eta_{0} \neq 0$. For this, we have for any $\phi \in D\left(H_{0}\right)$,

$$
\left\langle\left(H_{0}-E\right) \phi, \eta_{0}\right\rangle=\lim _{\epsilon \rightarrow 0}\left\langle\left(H_{0}-E\right) \phi, \eta_{\epsilon}\right\rangle=\langle\phi, C \eta\rangle \text {. }
$$

Since $D\left(H_{0}\right)$ is dense and $C \eta \neq 0$, this shows that $\eta_{0} \neq 0$. It follows from (3.74) that

$$
\begin{aligned}
\left\langle C^{*} \phi, \eta\right\rangle & =-\lambda\left\langle C^{*} \phi, \Gamma(E+i 0) \eta\right\rangle \\
& =-\lim _{\epsilon \rightarrow 0} \lambda\left\langle C^{*} \phi, D^{*} \eta_{\epsilon}\right\rangle \\
& =-\lambda\left\langle\phi, V \eta_{0}\right\rangle .
\end{aligned}
$$

Inserting this equation into (3.75), we obtain

$$
\left\langle\left(H_{\lambda}-E\right) \phi, \eta_{0}\right\rangle=0,
$$

which holds for all $\phi \in D\left(H_{0}\right)=D\left(H_{\lambda}\right)$. It follows that $\left(H_{\lambda}-E\right) \eta_{0}=0$, so that the energy $E$ is an eigenvalue of $H_{\lambda}$. Let us recall that the number of eigenvalues of $H_{\lambda}$ is countable. Hence, for each $\lambda \in \mathbb{R}$, there exists a subset $I_{\lambda} \subset I_{0}$, with $\left|I_{\lambda}\right|=\left|I_{0}\right|$ so that $n-\lim _{\epsilon \rightarrow 0}(1+\lambda \Gamma(E+i \epsilon))^{-1}$ exists for all $E \in I_{\lambda}$.

3. Using the second resolvent equation as in (3.71), we have for all $E \in I_{\lambda}$,

$$
R_{\lambda}(E+i \epsilon) C=R_{0}(E+i \epsilon) C(1+\lambda \Gamma(E+i \epsilon))^{-1} .
$$

By the result of part (2) and (A2), which controls the boundary value of $R_{0}(E+i \epsilon) C$, we obtain

$$
\sup _{\epsilon>0}\left\|R_{\lambda}(E+i \epsilon) C\right\|<C(E)<\infty, \forall E \in I_{\lambda} .
$$

Hence, we have obtained control on the boundary values of the resolvent of the perturbed operator given control on the boundary values of the unperturbed operator as in (A2).

4. We now exploit this control in order to make conclusions about the spectrum of $H_{\lambda}$ in $I$. First, with regards to the absolutely continuous spectrum, the existence of the bound (3.79) implies that for all $\phi \in \mathcal{H}$, and for all $E \in I_{\lambda}$,

$$
\lim _{\epsilon \rightarrow 0} \operatorname{Im}\left\langle\phi, C^{*} R_{\lambda}(E+i \epsilon) C \phi\right\rangle=0 .
$$


This proves $\sigma_{a c}\left(\tilde{H}_{\lambda}\right) \cap I=\emptyset$. Concerning the point spectrum in $I_{0}$, we know that the countably-many points $E \in I_{0}$ where the limit in (3.73) does not exist are eigenvalues of $\tilde{H}_{\lambda}$. At the other points in $I_{0}$, the bound (3.79) holds and, as in Theorem 3.3, this set cannot support the singular continuous component of the spectral measure of $\tilde{H}_{\lambda}$. This proves the first part of the theorem.

5. As for singular continuous spectrum, it can only be supported in $I \backslash I_{0}$, which has $\left|I \backslash I_{0}\right|=0$. We now use Corollary 3.2 taking $h(\lambda)=\left(1+\lambda^{2}\right)^{-1}$ (one can check that this type of function can be used in the proof). Since we have $C= \pm D$, we set $B=C$ in Corollary 3.2 and obtain,

$$
\left\|\int \frac{d \lambda}{1+\lambda^{2}} C E_{\lambda}\left(\left|I \backslash I_{0}\right|\right) C\right\| \leq C\left|I \backslash I_{0}\right|=0 .
$$

Consequently, for Lebesgue almost-every $\lambda \in \mathbb{R}$, we have that $\left\|C E_{\lambda}\left(I \backslash I_{0}\right) C\right\|=$ 0 . Due to the generating nature of the operator $C$, this proves that the singular component of the spectral measure of $\tilde{H}_{\lambda}$ is not supported on $I \backslash I_{0}$. Accordingly, the singular spectrum of $\tilde{H}_{\lambda}$ in $I$ consists only of eigenvalues in $I_{0}$, for almostevery $\lambda \in \mathbb{R}$. The finite degeneracy of the eigenvalues follows from compactness of $\Gamma(E+i 0)$.

\section{The Wegner Estimate and the Integrated Density of States}

4.1. Overview. The term Wegner estimate refers to an upper bound on the probability that a given energy $E$ is separated from the spectrum of a local Hamiltonian $H_{\Lambda}$. Equivalently, this is the probability that the local Hamiltonian $H_{\Lambda}$ has an eigenvalue in a given interval. A good Wegner estimate is one for which the upper bound depends linearly on the volume $|\Lambda|$ and on the distance from the energy $E$ to the spectrum of $H_{\Lambda}$. In this chapter, we will present two proofs of Wegner's estimate in order to illustrate the ideas and difficulties involved. Wegner's original proof [136] introduced the clever device of interchanging differentiation with respect to energy with differentiation with respect to the random variables on which the random potential depends. Since an expectation is taken in the course of the proof, these derivatives can be removed by an integration-by-parts with respect to the random variables. In some way, all subsequent proofs of the Wegner estimate depend on this trick.

We begin in section 1 by sketching a proof of Wegner's estimate for energies in a spectral gap of $H_{0}$ which follows the original argument. The proof is transparent but unfortunately leads to a $|\Lambda|^{2}$-volume dependence. This is sufficient for the proof of Anderson localization but provides no information in the IDS. We then give a simple version of the proof of a good Wegner's estimate from [28] that works for a wide variety of models at all energies. We will show how this implies the Lipschitz continuity of the integrated density of states (IDS). We remark that one can use the spectral shift function (SSF) (cf. $[\mathbf{2 7}, \mathbf{7 8}]$ ) in the proof of Wegner's estimate, but the result is not as strong.

We mention that the Wegner estimate discussed here depends crucially on the fact that the single-site potential $u$ is sign-definite. There have been some extensions to the nonsign-definite case, see $[\mathbf{7 1}, \mathbf{1 2 9}]$, and the review $[\mathbf{1 3 0}]$ for related results. The nonsign-definite case is important for applications to the proof of Anderson localization for Schrödinger operators with random vector potentials $[\mathbf{6 8}, \mathbf{7 1}, \mathbf{7 2}]$. 
4.2. Wegner's Argument: A First Proof of Wegner's Estimate. Here we give a proof of Wegner's estimate for energies in the gap of the unperturbed operator $H_{0}$. The proof of the Wegner estimate begins by replacing the probability with an expectation which, in general, is easier to compute. Let us assume that $H_{0}$ has a spectral gap $G$ and that $E \in G$. Since the local potential $V_{\Lambda}$ is a relatively compact perturbation of $H_{0}$, its effect is to introduce at most finitely-many eigenvalues $E_{j}(\Lambda)$ into the gap $G$. We label these eigenvalues in increasing order, including multiplicity. Let $\eta>0$ be chosen so that $I_{\eta} \equiv[E-\eta / 2, E+\eta / 2] \subset G$. We want to estimate

$$
\mathbb{P}\left\{\operatorname{dist}\left(E, \sigma\left(H_{\Lambda}\right)\right)<\eta\right\} .
$$

This probability is expressible in term of the finite-rank spectral projector for the interval $I_{\eta}$ and $H_{\Lambda}$, which we write as $E_{\Lambda}\left(I_{\eta}\right)$. This projection is a random variable, depending on the process restricted to the region $\Lambda$ in the iid case, but we suppress this in the notation. We have

$$
\mathbb{P}_{\Lambda}\left\{\operatorname{dist}\left(E, \sigma\left(H_{\Lambda}\right)\right)<\eta\right\}=\mathbb{P}_{\Lambda}\left\{\operatorname{Tr}\left(E_{\Lambda}\left(I_{\eta}\right)\right) \geq 1\right\} .
$$

We recall that Chebyshev's inequality for a random variable $X$ is

$$
\mathbb{P}\{X \geq \eta\} \leq \frac{1}{\eta} \mathbb{E}\{X\} .
$$

We apply this to the random variable $\operatorname{Tr}\left(E_{\Lambda}\left(I_{\eta}\right)\right)$ and obtain

$$
\mathbb{P}_{\Lambda}\left\{\operatorname{dist}\left(E, \sigma\left(H_{\Lambda}\right)\right)<\eta\right\} \leq \mathbb{E}_{\Lambda}\left\{\operatorname{Tr}\left(E_{\Lambda}\left(I_{\eta}\right)\right)\right\} .
$$

We now proceed to estimate the expectation of the trace on the right in (4.4). We follow the original argument of Wegner [136] as modified by Kirsch [83] and using some results of $[\mathbf{8 6}]$. Let $\rho$ be a smooth, monotone increasing function such that $\rho(x)=1$, for $x>\eta / 2$, and $\rho(x)=0$, for $x<-\eta / 2$. We choose $E \in G$ as above and $\eta$ such that $[E-3 \eta / 2, E+3 \eta / 2] \subset G$. By the functional calculus, we have

$\rho\left(H_{\Lambda}-E+\eta\right)-\rho\left(H_{\Lambda}-E-\eta\right)=\sum_{j}\left\{\rho\left(E_{j}(\Lambda)-E+\eta\right)-\rho\left(E_{j}(\Lambda)-E-\eta\right)\right\} P_{j}(\Lambda)$,

where the sum is over eigenvalues $E_{j}(\Lambda)$ of $H_{\Lambda}$ in $[E-3 \eta / 2, E+3 \eta / 2]$. The operators $P_{j}(\Lambda)$ are the projectors onto the corresponding eigenspaces. The difference of the two operators is therefore trace class. Note that this difference $\rho\left(H_{\Lambda}-E+\eta\right)-$ $\rho\left(H_{\Lambda}-E-\eta\right)$ is, roughly, the number of eigenvalues of $H_{\Lambda}$ less than $E+3 \eta / 2$ minus the number of eigenvalues of $H_{\Lambda}$ less than $E-3 \eta / 2$. However, the operator $H_{\Lambda}$ may have continuous spectrum to the left of $E-3 \eta / 2$ so the operator $\rho\left(H_{\Lambda}-E-\eta\right)$ by itself is not trace class.

The coefficient in (4.5) is always nonnegative and precisely equal to one for $E_{j}(\Lambda) \in I_{\eta}$, so we have

$$
\operatorname{Tr}\left(E_{\Lambda}\left(I_{\eta}\right)\right) \leq \operatorname{Tr}\left(\rho\left(H_{\Lambda}-E+\eta\right)-\rho\left(H_{\Lambda}-E-\eta\right)\right) .
$$

Thus, the right side will give us an upper bound for the right side of (4.4). There is one other advantage to the introduction of the smooth function $\rho$. The counting function for eigenvalues is not differentiable since it is a step function. We will use the differentiability of $\rho$ below. 
Returning to the expectation of the trace in (4.4), we can now bound it above by

$$
\mathbb{E}_{\Lambda}\left\{\operatorname{Tr}\left[\rho\left(H_{\Lambda}-E+\eta\right)-\rho\left(H_{\Lambda}-E-\eta\right)\right]\right\} \leq \mathbb{E}_{\Lambda}\left\{\operatorname{Tr}\left[\int_{-\eta}^{\eta} \frac{d}{d t} \rho\left(H_{\Lambda}-E-t\right)\right]\right\}
$$

Since the support of $\rho^{\prime}(s)$ lies in the interval $[-\eta / 2, \eta / 2]$, we write

$$
\operatorname{Tr}\left\{\rho^{\prime}\left(H_{\Lambda}-E-t\right)\right\}=\sum_{j} \rho^{\prime}\left(E_{j}(\Lambda)-E-t\right)
$$

where, as above, the sum is over the eigenvalues of $H_{\Lambda}$ in the interval $[E-3 \eta / 2, E+$ $3 \eta / 2$ ] including multiplicity. The first part of Wegner's trick is to consider the eigenvalues as functions of the random coupling constants and rewrite the differentiation with respect to the energy $t$ in terms of differentiation with respect to the coupling constants $\lambda_{j} \in \tilde{\Lambda}$. By the chain rule for differentiation, we have

$$
\sum_{k \in \tilde{\Lambda}} \frac{\partial}{\partial \lambda_{k}} \rho\left(E_{j}(\Lambda)-E-t\right)=\rho^{\prime}\left(E_{j}(\Lambda)-E-t\right) \sum_{k \in \tilde{\Lambda}} \frac{\partial}{\partial \lambda_{k}} E_{j}(\Lambda) .
$$

In the next step, we need to estimate the sum $\sum_{k \in \tilde{\Lambda}} \frac{\partial}{\partial \lambda_{k}} E_{j}(\Lambda)$ from below so we can solve (4.9) for an upper bound on $\rho^{\prime}\left(E_{j}(\Lambda)-E-t\right)$. We use the FeynmanHellman formula for the variation of an eigenvalue with respect to a parameter.

TheOREM 4.1. (Feynman-Hellman Theorem.) Let $H(s)$ be a one-parameter family of self-adjoint operators for $s \in I$, a neighborhood of zero. Suppose that $H(s)$ has a simple eigenvalue $E(s) \in C^{1}(I)$ with eigenfunction $\phi(s) \in C^{1}(I)$. We then have

$$
\frac{d}{d s} E(s)=\left\langle\phi(s),\left(\frac{d}{d s} H(s)\right) \phi(s)\right\rangle .
$$

Proof: We begin with the simple identity, valid for $s \in I$,

$$
0=\langle\phi(s),(H(s)-E(s)) \phi(s)\rangle,
$$

and differentiate each side. Note that the eigenvalue equation implies that

$$
0=\left\langle\frac{d}{d s} \phi(s),(H(s)-E(s)) \phi(s)\right\rangle,
$$

and similarly for the conjugate term. Since $\|\phi(s)\|=1$, we obtain the result from the term involving $\frac{d}{d s}(H(s)-E(s))$.

We will use this identity again in chapter 5 . The theorem is particularly useful in cases of analytic perturbation theory for which the hypotheses of the theorem are satisfied.

Returning to our discussion of the Wegner estimate, we apply the FeynmanHellman Theorem to the eigenvalues $E_{j}(\Lambda)$, which depend on the coupling constants $\lambda_{k}, k \in \tilde{\Lambda}$, and obtain

$$
\sum_{k \in \tilde{\Lambda}} \frac{\partial}{\partial \lambda_{k}} E_{j}(\Lambda)=\sum_{k \in \tilde{\Lambda}}\left\langle\phi_{j}, \frac{\partial}{\partial \lambda_{k}} H_{\Lambda} \phi_{j}\right\rangle .
$$

The Anderson-type potential depends linearly on the coupling constants so that

$$
\frac{\partial H_{\Lambda}}{\partial \lambda_{k}}=u_{k}
$$


It follows from this and (4.12) that we need to estimate from below the sum

$$
\sum_{k \in \tilde{\Lambda}}\left\langle\phi_{j}, u_{k} \phi_{j}\right\rangle
$$

for an eigenfunction $\phi_{j}$ of $H_{\Lambda}$.

Let us now suppose for simplicity that the single-site potential $u$ is bounded and has support in $B_{R_{0}}(0) \subset \Lambda_{1}(0)$. We assume that $u \geq 0$ and satisfies $u \geq \epsilon_{1}$ on $B_{R_{1}}(0) \subset \Lambda_{1}(0)$ for some $\epsilon_{1}>0$ and some $R_{1}>0$. Let us define a subset of $\Lambda$ by $\Lambda^{\prime} \equiv \cup_{k \in \tilde{\Lambda}} B_{R_{1}}(k)$. We then have

$$
\sum_{k \in \tilde{\Lambda}} u_{k} \geq \epsilon_{1} \chi_{\Lambda^{\prime}}
$$

We estimate from below the quantity $\left\langle\phi_{j}, \chi_{\Lambda^{\prime}} \phi_{j}\right\rangle$ using the Comparison Theorem of Kirsch, Stollmann, and Stolz (KSS) [86]. Let $H_{0}$ be a background Hamiltonian with an open spectral gap $G$, which is perturbed by a localized potential $V_{\Lambda}$. For any region $\mathcal{O} \subset \mathbb{R}^{d}$, let $\chi_{\mathcal{O}}$ be the characteristic function of the region.

THEOREM 4.2. Let $H_{0}$ and $V_{\Lambda}$ be as above and $H_{\Lambda} \phi=E \phi$ with $E \in G$ and $\phi \in L^{2}\left(\mathbb{R}^{d}\right)$. Suppose that the following two conditions are satisfied:

(1) There exists a potential $V_{0}$ such that, with $H_{\Lambda}^{0} \equiv H_{0}+V_{0}$, we have $E \in$ $\rho\left(H_{\Lambda}^{0}\right)$;

(2) There exists a subset $F \subset \Lambda$ and a constant $\theta>0$ so that dist $(F \cup$ $\left.\Lambda^{c},\left\{x \mid V_{\Lambda}(x) \neq V_{0}(x)\right\}\right)>\theta>0$.

Then, there exists a constant $C_{0}$, depending on dist $\left(E, \sigma\left(H_{0}\right)\right)^{-1}$, such that

$$
\|\phi\| \leq C_{0}\left(1+\left\|\left(H_{\Lambda}^{0}-E\right)^{-1} W_{1}\right\|\right)\left\|\chi_{F} \phi\right\|,
$$

where $W_{1} \equiv\left[H_{0}, \chi_{1}\right]$. Here, $\chi_{1}$ is a smoothed characteristic function of compact support with $\chi_{1}=1$ on $\operatorname{supp}\left(1-\chi_{F}\right)$, and $\chi_{1}=0$ on $\mathcal{D} \equiv\left\{x \mid V_{\Lambda}(x) \neq V_{0}(x)\right\}$.

Referring to this theorem, we must construct a comparison potential $V_{0}$. We do this as follows. We choose a radius $0<R_{2}<R_{1}$ and an $\epsilon_{2} \geq \epsilon_{1}$ so that $u \mid B_{R_{2}}(0)^{c} \leq \epsilon_{2}$. We define a subset of $\Lambda$ by $\mathcal{D} \equiv \cup_{k \in \tilde{\Lambda}} B_{R_{2}}(k)$. We define the comparison potential $V_{0}$ as follows

$$
\begin{aligned}
V_{0}(x) & =V_{\Lambda}(x) \text { for } x \in \mathcal{D}^{c} \cap \Lambda \\
& =0 \text { for } x \in \mathcal{D}
\end{aligned}
$$

With this definition, we can take the set $F$ to be $F \equiv\left(\Lambda^{\prime}\right)^{c} \cap \Lambda$. Note that the distance from the set $\mathcal{D}$ on which the potentials are not equal to $F$ is $\left(R_{1}-R_{2}\right)>0$. Furthermore, we have $\left|V_{0}(x)\right| \leq \epsilon_{2}$. Consequently, the spectrum of $H_{\Lambda}^{0}$ is contained in the complement of the gap $\tilde{G} \equiv\left(B_{-}+\epsilon_{2}, B_{+}-\epsilon_{2}\right)$. Given an energy $E \in G$ and an $\eta$ as above, we choose $R_{2}$ and $R_{1}$ sufficiently close to $R_{0}$ so that $\epsilon_{2}$ is sufficiently small in order to guarantee that the $3 \eta / 2$-interval around $E$ does not intersect the gap $\tilde{G}$. This insures that any eigenvalue of $H_{\Lambda}$ in the interval is not in the spectrum of the comparison operator $H_{\Lambda}^{0}$.

Having verified all the hypotheses of the KSS Comparison Theorem, we obtain the estimate

$$
\left\langle\phi_{j}, \chi_{\tilde{\Lambda}} \phi_{j}\right\rangle \geq C_{0}\left\|\phi_{j}\right\|^{2}
$$


for a constant depending on dist $\left(E, \sigma\left(H_{0}\right)\right)$. Returning to (4.13), we have the lower bound,

$$
\sum_{k \in \tilde{\Lambda}} \frac{\partial E_{j}(\Lambda)}{\partial \lambda_{k}} \geq C_{0}
$$

so we obtain

$$
\rho^{\prime}\left(E_{j}(\Lambda)-E-t\right) \leq \frac{1}{C_{0}} \sum_{k \in \tilde{\Lambda}} \frac{\partial \rho}{\partial \lambda_{k}}\left(E_{j}(\Lambda)-E-t\right) .
$$

With this estimate, the right side of (4.7) can be bounded above by

$$
\frac{1}{C_{0}} \sum_{j} \sum_{k \in \tilde{\Lambda}} \int_{-\eta}^{\eta} \mathbb{E}_{\Lambda}\left\{\frac{\partial \rho}{\partial \lambda_{k}}\left(E_{j}(\Lambda)-E-t\right)\right\} d t
$$

The expectation is the integral over the random variables $\lambda_{l}$ with the product measure. Let us integrate over one of these variables, say the $k^{t h}$ one. Because of the positivity of $\rho^{\prime}$ and of the density $g$, we obtain

$$
\int d \lambda_{k} g\left(\lambda_{k}\right) \frac{\partial \rho}{\partial \lambda_{k}}\left(E_{j}(\Lambda)-E-t\right) \leq\|g\|_{\infty}\left\{\rho\left(E_{j}^{M, k}(\Lambda)-e-t\right)-\rho\left(E_{j}^{m, k}(\Lambda)-E-t\right)\right\},
$$

where $E_{j}^{M, k}$ is the $j^{t h}$-eigenvalue of the local Hamiltonian $H_{\Lambda}$ with the coupling constant at the $k^{t h}$-site fixed at its maximum value. Similarly, the small $m$ denotes the minimum value. Consequently, we are left with the task of estimating

$$
\frac{\|g\|_{\infty}}{C_{0}} \sum_{k \in \tilde{\Lambda}} \int_{-\eta}^{\eta} d t \int \Pi_{l \neq k} g\left(\lambda_{l}\right) d \lambda_{l} \operatorname{Tr}\left\{\rho\left(H_{\Lambda}^{M, k}-E-t\right)-\rho\left(H_{\Lambda}^{m, k}-E-t\right)\right\} .
$$

The expression involving the trace is basically the number of eigenvalues created in the interval by increasing the $k^{t h}$-coupling constant from the minimum to the maximum value. In fact, the trace can be rewritten in terms of the spectral shift function (SSF). Estimates on the SSF are key to a good Wegner estimate and we will discuss this in the next section. Let us note here that Weyl's upper bound for the eigenvalue counting function gives a crude estimate in terms of the volume:

$$
\left|\operatorname{Tr}\left\{\rho\left(H_{\Lambda}^{M, k}-E-t\right)-\rho\left(H_{\Lambda}^{m, k}-E-t\right)\right\}\right| \leq C_{1}|\Lambda| .
$$

Finally, this estimate and equations (4.4) and (4.24), lead us to the result

$$
\mathbb{P}\left\{\operatorname{dist}\left(E, \sigma\left(H_{\Lambda}\right)\right)<\eta\right\} \leq 2 \eta \frac{\|g\|_{\infty} C_{1}}{C_{0}}|\Lambda|^{2} .
$$

This estimate is sufficient for the proof of localization for the models we will discuss.

There are two defects in the simple proof sketched above. First, the restriction to the spectral gap of $H_{0}$ was necessary for the crucial lower bound estimate (4.19) following from Theorem 4.2. This lower bound is a form of the unique continuation theorem for solutions to elliptic equations. Roughly, if the left side of (4.19) is zero, this means that the eigenfunction $\phi_{j}$ vanishes on an open set. However, if $\phi_{j}$ were analytic, this would imply that $\phi_{j}=0$ identically. A refined and quantitative version of this type of result will allow us to get a good lower bound without using Theorem 4.2. Secondly, the crude estimate on (4.25) is too big. The upper bound should depend on the size of the support of the perturbation $u$, not the total volume $|\Lambda|$. 
4.3. The Wegner Estimate: Preliminaries. The optimal form of the Wegner estimate has an upper bound that depends on the first power of the volume and the first power of the length of the interval. This first power of the volume will insure that the thermodynamic limit exists, whereas the first power of the interval size will prove the Lipschitz continuity of the IDS via the bound, for $E \leq E^{\prime}$,

$$
0 \leq N\left(E^{\prime}\right)-N(E) \leq \liminf _{\mid \Lambda \rightarrow \infty} \mathbb{E}\left\{\operatorname{Tr}\left(E_{\Lambda}\left(\left[E, E^{\prime}\right]\right)\right\} .\right.
$$

We will prove, then, for any small energy interval,

$$
\mathbb{E}\left\{\operatorname{Tr} E_{\Lambda}\left(\left[E, E^{\prime}\right]\right)\right\} \leq C_{I}\left|E-E^{\prime} \| \Lambda\right|,
$$

for some locally uniform constant $C_{I}$. In order to prove the Lipschitz continuity, we will assume that the single-site probability distribution has a bounded density with compact support. Much more general situations are discussed in [28].

In order to control the background operator, we make the assumption that $H_{0}=\left(-i \nabla-A_{0}\right)^{2}+V_{0}$ is a periodic Schrödinger operator with a real-valued, periodic, potential $V_{0}$, and a periodic vector potential $A_{0}$. We assume that $V_{0}$ and $A_{0}$ are sufficiently regular so that $H_{0}$ is essentially self-adjoint on $C_{0}^{\infty}\left(\mathbb{R}^{d}\right)$. We assume that both $V_{0}$ and $A_{0}$ are periodic with respect to the group $\Gamma=\mathbb{Z}^{d}$ because of the form of the Anderson-type potential. We note that we could work with a nondegenerate lattice $\Gamma$, by defining a corresponding Anderson-type potential, but we will explicitly treat the case $\Gamma=\mathbb{Z}^{d}$.

Concerning the Anderson-type random potential, We will always make the following four assumptions:

(A1): The background operator $H_{0}=\left(-i \nabla-A_{0}\right)^{2}+V_{0}$ is a lower semibounded, $\mathbb{Z}^{d}$-periodic Schrödinger operator with a real-valued, $\mathbb{Z}^{d}$-periodic, potential $V_{0}$, and a $\mathbb{Z}^{d}$-periodic vector potential $A_{0}$. We assume that $V_{0}$ and $A_{0}$ are sufficiently regular so that $H_{0}$ is essentially self-adjoint on $C_{0}^{\infty}\left(\mathbb{R}^{d}\right)$.

(A2): The periodic operator $H_{0}$ has the unique continuation property (UCP), that is, for any $E \in \mathbb{R}$ and for any function $\phi \in H_{l o c}^{2}\left(\mathbb{R}^{d}\right)$, if $\left(H_{0}-E\right) \phi=$ 0 , and if $\phi$ vanishes on an open set, then $\phi \equiv 0$.

(A3): The nonzero, nonnegative, compactly-supported, single-site potential $u \in L_{0}^{\infty}\left(\mathbb{R}^{d}\right)$, and it is strictly positive on a nonempty open set.

(A4): The random coupling constants $\left\{\lambda_{j}(\omega) \mid j \in \mathbb{Z}^{d}\right\}$, are independent and identically distributed. The distribution has a density $h_{0} \in L^{\infty}(\mathbb{R})$ with $\operatorname{supp} h_{0} \subset[0,1]$.

We make three important comments on these hypotheses:

(1) For a given density $h_{0}$ of compact support $\left[m, M_{1}\right]$, we can always add the periodic potential $m \sum_{j} u_{j}$, with $u_{j}(x)=u(x-j)$, to the background potential $V_{0}$, so that the random coupling constants take their value in an interval $[0, M]$, with $M=M_{1}-m$.

(2) Because of the explicit disorder parameter $\lambda>0$, we can rescale the coupling constants so that, without loss of generality, the support of $h_{0}$ is included in the interval $[0,1]$.

(3) Hypotheses (A1) and (A2) imply the following. There exists a finite constant $C_{1}(u, M, d)>0$, depending only on the single-site potential $u$, and 
the dimension $d \geq 1$, so that for all $\Lambda \subset \mathbb{R}^{d}$,

$$
0 \leq V_{\Lambda}^{2} \leq C_{1}(u, d) \tilde{V}_{\Lambda}
$$

where $V_{\Lambda}$ and $\tilde{V}_{\Lambda}$ are defined in (1.3) and (1.4), respectively. This simple inequality is used in the proof of Theorem 4.3.

These hypotheses overlap with the hypotheses of chapter 1 . As we have discussed, the Wegner estimate and the continuity of the IDS are very closely related. We first give the result on the Wegner estimate.

TheOREM 4.3. Assume hypotheses (A1)-(A4). Let $\Delta \subset \mathbb{R}$ be a bounded, closed interval. Then, there exists a finite constant $C_{W}>0$, locally uniform in energy, and depending on $d, u$, and $\lambda>0$, so that

$$
\mathbb{E}\left\{\operatorname{Tr} E_{\Lambda}(\Delta)\right\} \leq C_{W}|\Lambda||\Delta| .
$$

We note that one can show that $C_{W} \sim 1 / \lambda$. It is possible to obtain a weaker Wegner estimate for which the constant $C_{W}$ is independent of $\lambda$ (cf. [27] and an application to continuity of the IDS with respect to the disorder in [73]). This has consequences for the continuity, with respect to the disorder $\lambda$ of the IDS [73]. Under the same conditions, we obtain the following Lipschitz continuity result on the IDS.

THEOREM 4.4. Assume hypotheses (A1)-(A4). Then, the IDS N(E) for the random family $H_{\omega}(\lambda)=H_{0}+\lambda V_{\omega}$, for $\lambda \neq 0$, is locally Lipschitz continuous on $\mathbb{R}$.

4.3.1. Quantitative Unique Continuation Principle. Before we sketch the proof of Theorem 4.3, we must discuss an important consequence of hypothesis (A2) on the background operator $H_{0}$ that we refer to as a quantitative UCP. We first recall the Floquet decomposition of a $\Gamma$-periodic operator. Let $\Gamma^{*}$ denote the dual lattice, that is, $\Gamma^{*}=\left\{\gamma^{\prime} \mid \gamma \cdot \gamma^{\prime} \in 2 \pi \mathbb{Z}\right.$, for all $\left.\gamma \in \Gamma\right\}$. We let $I^{d}=\mathbb{R}^{d} / \mathbb{Z}^{d}$ be the torus, and $\left(\Pi^{d}\right)^{*}=\left(\mathbb{R}^{d}\right)^{*} /\left(\mathbb{Z}^{d}\right)^{*}$ be the dual torus. We denote by $C_{0}$ the unit cell for $\Gamma=\mathbb{Z}^{d}$, and by $C_{0}^{*}$ the unit cell for $\Gamma^{*}=\left(\mathbb{Z}^{d}\right)^{*}$. The Floquet decomposition of $H_{0}$ yields a family of operators $H_{0}(\theta)$, for $\theta \in\left(\mathbb{R}^{d}\right)^{*}$. Each operator $H_{0}(\theta)$ is self-adjoint on $L^{2}\left(\Pi^{d}\right)$, and has a compact resolvent. We denote the eigenvalues of $H_{0}(\theta)$ by $E_{n}(\theta)$. The spectrum of $H_{0}$ is given by

$$
\sigma\left(H_{0}\right)=\bigcup_{\theta \in\left(\mathbb{T}^{d}\right)^{*}} \sigma\left(H_{0}(\theta)\right)=\bigcup_{n \in \mathbb{N}} \bigcup_{\theta \in\left(\mathbb{T}^{d}\right)^{*}} E_{n}(\theta) .
$$

For an open, relatively compact interval $\Delta \subset \mathbb{R}$, we let $E_{0}(\Delta, \theta)$ denote the spectral projector of $H_{0}(\theta)$ onto the eigenspace of $H_{0}(\theta)$ spanned by its eigenfunctions with eigenvalues $E_{n}(\theta) \in \Delta$. Because of the discreteness of the spectrum of $H_{0}(\theta)$, the dimension of $\operatorname{Ran} E_{0}(\Delta, \theta)$ is finite and locally constant.

We also need to consider $H_{0}$ as an $n \Gamma$-periodic operator for any $n \in \mathbb{I N}$. Let $H_{0}^{n}(\theta)$ be the operator $H_{0}$ restricted to the torus $\mathbb{R}^{d} /(n \Gamma)$, with $\theta$-quasi-periodic boundary conditions. For $I \subset \mathbb{R}$, an interval, let $\mathbf{E}_{0}^{n}(I, \theta)$ denote the spectral projection onto the interval $I$ for $H_{0}^{n}(\theta)$. We remark that in section 2 we took $I=\tilde{\Delta}$. Finally, for any $\Gamma$-periodic function $g$, we write $g^{(n)}$ for the same function understood as an $n \Gamma$-periodic function.

Theorem 4.5. Let $V: \mathbb{R}^{d} \rightarrow \mathbb{R}$ be a bounded, real-valued, $\Gamma$-periodic function. Consider a bounded interval $I \subset \mathbb{R}$. Then, if there exists a finite constant $C(I, V)>$ 
0 such that, for all $\theta \in \mathbb{R}^{d}$, one has

$$
\mathbf{E}_{0}(I, \theta) V \mathbf{E}_{0}(I, \theta) \geq C(I, V) \mathbf{E}_{0}(I, \theta),
$$

then, for all $n \geq 1$ and all $\theta \in \mathbb{R}^{d}$, one has, with the same constant $C(I, V)$,

$$
\mathbf{E}_{0}^{n}(I, \theta) V^{(n)} \mathbf{E}_{0}^{n}(I, \theta) \geq C(I, V) \mathbf{E}_{0}^{n}(I, \theta),
$$

where $V$ is considered as a $\Gamma$-periodic function in (4.1), and $V^{(n)}$ represents the corresponding $n \Gamma$-periodic function in (4.2).

We next show that, in fact, condition (4.1) holds for a wide family of periodic potentials $V$. We remind the reader that in the applications, we will take the potential $V$ appearing in (4.1) to be the single-site potential $u$, restricted to the unit cell $\Lambda_{1}(0)$, viewed as a $\Gamma$-periodic function. The $n \Gamma$-periodic function appearing in $(4.2)$ is $\tilde{V}(x)=\sum_{j} u(x-j)$, restricted to $\Lambda^{(n)}$, where $\Lambda^{(n)}$ is the basic $n \Gamma$-periodic cell.

TheOREM 4.6. Let $V: \mathbb{R}^{d} \rightarrow \mathbb{R}$ be a bounded, $\Gamma$-periodic, nonnegative function. Suppose that $V>0$ on some open set and $H_{0}$ has the unique continuation property. Then, condition (4.1) holds for any compact interval $I \subset \mathbb{R}$ with a finite constant $C(I, V)>0$.

As a summary of these results, we have the following. Let $\Lambda \subset \mathbb{R}^{d}$ be a $\mathbb{Z}^{d_{\text {- }}}$ periodic cell. There exists a finite constant $C_{0}>0$, depending only on $u$ and the dimension $d$, so that for any closed, bounded interval $I \subset \mathbb{R}$, we have

$$
E_{0}^{\Lambda}(I) \tilde{V}_{\Lambda} E_{0}^{\Lambda}(I) \geq C_{0} E_{0}^{\Lambda}(I)
$$

This is the quantitative UCP. It plays a key role in the proof of Theorem 4.3.

4.3.2. Spectral Averaging. The second ingredient is the spectral averaging result, Theorem 3.1 of chapter 3 , and its Corollary 3.1. We use this estimate (3.43) as follows. Let $E_{0} \in \mathbb{R}$ be fixed and arbitrary. We consider an interval $\Delta_{\epsilon}=$ $\left[E_{0}, E_{0}+\epsilon\right]$, for some fixed $0<\epsilon<\infty$. A simple use of the spectral theorem for a self-adjoint operator $H$ with spectral family $E_{H}(\cdot)$ shows that

$$
\begin{aligned}
\int_{\Delta_{\epsilon}} & d E\left\langle\phi, \Im(H-E-i \epsilon)^{-1} \phi\right\rangle \\
= & \left\langle\phi,\left[\tan ^{-1}\left(\frac{E_{0}+\epsilon-H}{\epsilon}\right)-\tan ^{-1}\left(\frac{E_{0}-H}{\epsilon}\right)\right] \phi\right\rangle \\
& \geq\left(\tan ^{-1} 1\right)\left\langle\phi, E_{H}\left(\Delta_{\epsilon}\right) \phi\right\rangle=(\pi / 4)\left\langle\phi, E_{H}\left(\Delta_{\epsilon}\right) \phi\right\rangle,
\end{aligned}
$$

so that

$$
\left\langle\phi, E_{H}\left(\Delta_{\epsilon}\right) \phi\right\rangle \leq \frac{4}{\pi} \int_{\Delta_{\epsilon}} d E\left\langle\phi, \Im(H-E-i \epsilon)^{-1} \phi\right\rangle .
$$

We combine (4.36) with Theorem 3.1 to obtain

$$
\mathbb{E}\left\{\left\langle\phi, u_{j} E_{\Lambda}(\Delta) u_{j} \phi\right\rangle\right\} \leq 8|\epsilon|\|\phi\|^{2} .
$$

We remark that Theorem 3.3 leads to a similar upper bound for more general probability distributions with the factor $\epsilon$ replaced by the Levy concentration $s(\epsilon)$ of the probability measure as described in (3.35) and Corollary (3.1), see also [28]. 
4.4. Proof of Theorem 4.3. 1. As in section 2, we need to estimate

$$
\mathbb{E}\left\{\operatorname{Tr} E_{\Lambda}(\Delta)\right\} .
$$

We begin with a decomposition relative to the spectral projectors $E_{0}^{\Lambda}(\cdot)$ for the operator $H_{0}^{\Lambda}$. We write

$$
\operatorname{Tr} E_{\Lambda}(\Delta)=\operatorname{Tr} E_{\Lambda}(\Delta) E_{0}^{\Lambda}(\tilde{\Delta})+\operatorname{Tr} E_{\Lambda}(\Delta) E_{0}^{\Lambda}\left(\tilde{\Delta}^{c}\right) .
$$

We require $\Delta \subset \tilde{\Delta}$ satisfy $|\Delta|<1$ and that the distance from the interval $\Delta$ to the unbounded set $\tilde{\Delta}^{c}$ be strictly positive, that is, $d_{\Delta}>0$. If $\tilde{\Delta}$, and consequently $\Delta$, lies in a spectral gap of $H_{0}$, then only the second term on the right in (4.39) contributes and the result follows from (4.49). Hence, we only need to consider the case when $\Delta$ does not lie in a spectral gap of $H_{0}$.

2. The term involving $\tilde{\Delta}^{c}$ is estimated as follows. Since $E_{\Lambda}(\Delta)$ is trace class, let $\left\{\phi_{m}^{\Lambda}\right\}$ be the set of normalized eigenfunctions in its range. We expand the trace in these eigenfunctions and obtain

$$
\operatorname{Tr} E_{\Lambda}(\Delta) E_{0}^{\Lambda}\left(\tilde{\Delta}^{c}\right)=\sum_{m}\left\langle\phi_{m}^{\Lambda}, E_{0}^{\Lambda}\left(\tilde{\Delta}^{c}\right) \phi_{m}^{\Lambda}\right\rangle .
$$

From the eigenfunction equation $\left(H_{\omega}^{\Lambda}-E_{m}\right) \phi_{m}^{\Lambda}=0$, we easily obtain

$$
-\left(H_{0}^{\Lambda}-E_{m}\right)^{-1} E_{0}^{\Lambda}\left(\tilde{\Delta}^{c}\right) V_{\Lambda} \phi_{m}^{\Lambda}=E_{0}^{\Lambda}\left(\tilde{\Delta}^{c}\right) \phi_{m}^{\Lambda} .
$$

Substituting this into the right side of (4.40), and resumming to obtain a trace, we find

$$
\operatorname{Tr} E_{\Lambda}(\Delta) E_{0}^{\Lambda}\left(\tilde{\Delta}^{c}\right)=\sum_{m}\left\langle\phi_{m}^{\Lambda},\left(V_{\Lambda} \frac{E_{0}^{\Lambda}\left(\tilde{\Delta}^{c}\right)}{\left(H_{0}^{\Lambda}-E_{m}\right)^{2}} V_{\Lambda}\right) \phi_{m}^{\Lambda}\right\rangle .
$$

We next want to replace the energy $E_{m} \in \Delta$ in the resolvent in (4.41) by a fixed number, say $-M$, assuming $H_{0}^{\Lambda}>-M>-\infty$. To do this, we define an operator $K$ by

$$
K \equiv\left(\frac{H_{0}^{\Lambda}+M}{H_{0}^{\Lambda}-E_{m}}\right)^{2} E_{0}^{\Lambda}\left(\tilde{\Delta}^{c}\right),
$$

and note that $K$ is bounded, independent of $m$, by

$$
\|K\| \leq K_{0} \equiv\left[1+\frac{2\left(M+\Delta_{+}\right)}{d_{\Delta}}+\frac{\left(M+\Delta_{+}\right)^{2}}{d_{\Delta}^{2}}\right],
$$

where $\Delta=\left[\Delta_{-}, \Delta_{+}\right]$. Now, for any $\psi \in L^{2}\left(\mathbb{R}^{d}\right)$,

$$
\begin{aligned}
\left\langle\psi, \frac{E_{0}^{\Lambda}\left(\tilde{\Delta}^{c}\right)}{\left(H_{0}^{\Lambda}-E_{m}\right)^{2}} \psi\right\rangle & \leq\left\langle\frac{E_{0}^{\Lambda}\left(\tilde{\Delta}^{c}\right)}{\left(H_{0}^{\Lambda}+M\right)} \psi, K \frac{E_{0}^{\Lambda}\left(\tilde{\Delta}^{c}\right)}{\left(H_{0}^{\Lambda}+M\right)} \psi\right\rangle \\
& \leq K_{0}\left\langle\psi, \frac{E_{0}^{\Lambda}\left(\tilde{\Delta}^{c}\right)}{\left(H_{0}^{\Lambda}+M\right)^{2}} \psi\right\rangle \\
& \leq K_{0}\left\langle\psi, \frac{1}{\left(H_{0}^{\Lambda}+M\right)^{2}} \psi\right\rangle,
\end{aligned}
$$

since $E_{0}^{\Lambda}\left(\tilde{\Delta}^{c}\right) \leq 1$. We use the bound (4.43) on the right in (4.41) and expand the potential. To facilitate this, let $\chi \geq 0$ be a function of compact support slightly 
larger than the support of $u$, and so that $\chi u=u$. We set $\chi_{j}(x)=\chi(x-j)$, for $j \in \mathbb{Z}^{d}$. Returning to (4.41), we obtain the bound

$$
\begin{aligned}
\operatorname{Tr} E_{\Lambda}(\Delta) E_{0}^{\Lambda}\left(\tilde{\Delta}^{c}\right) & \leq K_{0} \operatorname{Tr} E_{\Lambda}(\Delta)\left(V_{\Lambda} \frac{1}{\left(H_{0}^{\Lambda}+M\right)^{2}} V_{\Lambda}\right) \\
& \leq K_{0} \sum_{i, j \in \tilde{\Lambda}}\left|\omega_{i} \omega_{j}\right|\left|\operatorname{Tr}\left[u_{j} E_{\Lambda}(\Delta) u_{i} \cdot\left(\chi_{i} \frac{1}{\left(H_{0}^{\Lambda}+M\right)^{2}} \chi_{j}\right)\right]\right| \\
& \leq K_{0} \sum_{i, j \in \tilde{\Lambda}}\left|\operatorname{Tr}\left[u_{j} E_{\Lambda}(\Delta) u_{i} \cdot\left(\chi_{i} \frac{1}{\left(H_{0}^{\Lambda}+M\right)^{2}} \chi_{j}\right)\right]\right|
\end{aligned}
$$

3. Let us make the simplifying assumption that $u_{i} u_{j}=0$, if $i \neq j$. This is the case when $u$ has support inside a unit cube. It is also the most difficult case. We refer to $[\mathbf{2 8}]$ for the general case. We note that the operator $K_{i j} \equiv \chi_{i}\left(H_{0}^{\Lambda}+M\right)^{-2} \chi_{j}$ in (4.41) is trace class for $d=1,2,3$. It is proved in [28] that the operator $K_{i j}$ is trace class in all dimensions when $\chi_{i} \chi_{j}=0$, and the trace norm $\left\|K_{i j}\right\|_{1}$ decays exponentially in $\|i-j\|$ as

$$
\left\|K_{i j}\right\|_{1}=\left\|\chi_{i}\left(H_{0}^{\Lambda}+M\right)^{-2} \chi_{j}\right\|_{1} \leq C_{0} e^{-c_{0}\|i-j\|}, i i \neq j,
$$

for positive constants $C_{0}, c_{0}>0$ depending on $M$. This exponential decay is crucial for controlling the double sum in (4.44) in order to obtain just one power of the volume. Omitting some technical details that arise in dimensions $d>3$, we write the trace on the last line of (4.44) as

$$
\sum_{i, j \in \tilde{\Lambda}} \operatorname{Tr}\left[u_{j} E_{\Lambda}(\Delta) u_{i} \cdot K_{i j}\right]
$$

with $K_{i j}$ trace class and satisfying the decay estimate (4.45). The canonical representation of $\tilde{K}(n)_{i j}$ (where we write $j$ for $j_{n}$ ) is

$$
\tilde{K}(n)_{i j}=\sum_{l} \mu_{l}^{(i j)}\left|\phi_{l}^{(i j)}\right\rangle\left\langle\psi_{l}^{(i j)}\right|
$$

where $\left(\phi_{l}^{(i j)}\right)_{l},\left(\psi_{l}^{(i j)}\right)_{l}$ are orthonormal families and $\sum_{l}\left|\mu_{l}^{(i j)}\right|<+\infty$.

Inserting this into (4.46), we obtain

$$
\begin{aligned}
\sum_{i, j \in \tilde{\Lambda}} \operatorname{Tr}\left[u_{j} E_{\Lambda}(\Delta) u_{i} \cdot K_{i j}\right] \leq & \sum_{i, j \in \tilde{\Lambda}} \sum_{l} \mu_{l}^{(i j)}\left\langle\psi_{l}^{(i j)}, u_{j} E_{\Lambda}(\Delta) u_{i} \phi_{l}^{(i j)}\right\rangle \\
\leq & \sum_{i, j \in \tilde{\Lambda}} \sum_{l} \mu_{l}^{(i j)}\left\{\left\langle\psi_{l}^{(i j)}, u_{j} E_{\Lambda}(\Delta) u_{j} \psi_{l}^{(i j)}\right\rangle+\right. \\
& \left.\left\langle\phi_{l}^{(i j)}, u_{i} E_{\Lambda}(\Delta) u_{i} \phi_{l}^{(i j)}\right\rangle\right\} .
\end{aligned}
$$

It follows from (4.37) that the expectation of the matrix elements in (4.47) satisfy the following bound

$$
\mathbb{E}\left\{\left\langle\psi_{l}^{(i j)}, u_{j} E_{\Lambda}(\Delta) u_{j} \psi_{l}^{(i j)}\right\rangle\right\} \leq 8|\Delta| .
$$

Returning to (4.44), we obtain

$$
\operatorname{Tr} E_{\Lambda}(\Delta) E_{0}^{\Lambda}\left(\tilde{\Delta}^{c}\right) \leq K_{0} C(u, m)|\Delta||\Lambda| .
$$


3. As for the first term on the right in (4.39), we use the fundamental assumption (4.34). As in $[\mathbf{2 5}]$, we will use the spectral projector $E_{0}(\tilde{\Delta})$ of $H_{0}^{\Lambda}$ in order to control the trace. We have

$$
\begin{aligned}
\operatorname{Tr} E_{\Lambda}(\Delta) E_{0}^{\Lambda}(\tilde{\Delta}) \leq & \frac{1}{C(\tilde{\Delta}, u)}\left\{\operatorname{Tr} E_{\Lambda}(\Delta) E_{0}^{\Lambda}(\tilde{\Delta}) \tilde{V}_{\Lambda} E_{0}^{\Lambda}(\tilde{\Delta})\right\} \\
\leq & \frac{1}{C(\tilde{\Delta}, u)}\left\{\operatorname{Tr} E_{\Lambda}(\Delta) \tilde{V}_{\Lambda} E_{0}^{\Lambda}(\tilde{\Delta})\right. \\
& \left.-\operatorname{Tr} E_{\Lambda}(\Delta) E_{0}^{\Lambda}\left(\tilde{\Delta}^{c}\right) \tilde{V}_{\Lambda} E_{0}^{\Lambda}(\tilde{\Delta})\right\} .
\end{aligned}
$$

We estimate the second term on the right in (4.50). Using the Hölder inequality for trace norms, we have, for any $\kappa_{0}>0$,

$$
\begin{aligned}
& \left|\operatorname{Tr} E_{\Lambda}(\Delta) E_{0}^{\Lambda}\left(\tilde{\Delta}^{c}\right) \tilde{V}_{\Lambda} E_{0}^{\Lambda}(\tilde{\Delta})\right| \\
& \quad \leq\left\|E_{\Lambda}(\Delta) E_{0}^{\Lambda}\left(\tilde{\Delta}^{c}\right)\right\|_{2}\left\|\tilde{V}_{\Lambda} E_{0}^{\Lambda}(\tilde{\Delta}) E_{\Lambda}(\Delta)\right\|_{2} \\
& \quad \leq \frac{1}{2 \kappa_{0}} \operatorname{Tr} E_{0}^{\Lambda}\left(\tilde{\Delta}^{c}\right) E_{\Lambda}(\Delta)+\frac{\kappa_{0}}{2} \operatorname{Tr} E_{\Lambda}(\Delta) E_{0}^{\Lambda}(\tilde{\Delta}) \tilde{V}_{\Lambda}^{2} E_{0}^{\Lambda}(\tilde{\Delta}) E_{\Lambda}(\Delta) .
\end{aligned}
$$

We next estimate the second term on the right in (4.51). Let $D_{0}$ be a finite constant so that $\tilde{V}_{\Lambda}^{2} \leq D_{0} \tilde{V}_{\Lambda}$. Using this, we find that for any $\kappa_{1}>0$,

$$
\begin{aligned}
& \operatorname{Tr} E_{\Lambda}(\Delta) E_{0}^{\Lambda}(\tilde{\Delta}) \tilde{V}_{\Lambda}^{2} E_{0}^{\Lambda}(\tilde{\Delta}) E_{\Lambda}(\Delta) \\
& \quad \leq D_{0}\left\|E_{\Lambda}(\Delta) E_{0}^{\Lambda}(\tilde{\Delta}) \tilde{V}_{\Lambda}\right\|_{2}\left\|E_{0}^{\Lambda}(\tilde{\Delta}) E_{\Lambda}(\Delta)\right\|_{2} \\
& \quad \leq \frac{D_{0} \kappa_{1}}{2} \operatorname{Tr} E_{\Lambda}(\Delta) E_{0}^{\Lambda}(\tilde{\Delta}) \tilde{V}_{\Lambda}^{2} E_{0}^{\Lambda}(\tilde{\Delta}) E_{\Lambda}(\Delta)+\frac{D_{0}}{2 \kappa_{1}} \operatorname{Tr} E_{\Lambda}(\Delta) E_{0}^{\Lambda}(\tilde{\Delta}) .
\end{aligned}
$$

We choose $\kappa_{1}=1 / D_{0}>0$ so that $\left(1-D_{0} \kappa_{1} / 2\right)=1 / 2$. Consequently, we obtain

$$
\operatorname{Tr} E_{\Lambda}(\Delta) E_{0}^{\Lambda}(\tilde{\Delta}) \tilde{V}_{\Lambda}^{2} E_{0}^{\Lambda}(\tilde{\Delta}) E_{\Lambda}(\Delta) \leq D_{0}^{2} \operatorname{Tr} E_{\Lambda}(\Delta) E_{0}^{\Lambda}(\tilde{\Delta}) .
$$

Inserting this into (4.51), we find

$$
\left|\operatorname{Tr} E_{\Lambda}(\Delta) E_{0}^{\Lambda}\left(\tilde{\Delta}^{c}\right) \tilde{V}_{\Lambda} E_{0}^{\Lambda}(\tilde{\Delta})\right| \leq \frac{1}{2 \kappa_{0}} \operatorname{Tr} E_{0}^{\Lambda}\left(\tilde{\Delta}^{c}\right) E_{\Lambda}(\Delta)+\frac{\kappa_{0} D_{0}^{2}}{2} \operatorname{Tr} E_{\Lambda}(\Delta) E_{0}^{\Lambda}(\tilde{\Delta}) .
$$

As a consequence of (4.53), we obtain for the first term on the right in (4.39),

$$
\begin{aligned}
& \left(1-\frac{\kappa_{0} D_{0}^{2}}{2 C(\tilde{\Delta}, u)}\right) \operatorname{Tr} E_{\Lambda}(\Delta) E_{0}^{\Lambda}(\tilde{\Delta}) \\
& \quad \leq \frac{1}{C(\tilde{\Delta}, u)}\left|\operatorname{Tr} E_{\Lambda}(\Delta) \tilde{V}_{\Lambda} E_{0}^{\Lambda}(\tilde{\Delta})\right|+\frac{1}{2 \kappa_{0} C(\tilde{\Delta}, u)} \operatorname{Tr} E_{\Lambda}(\Delta) E_{0}^{\Lambda}\left(\tilde{\Delta}^{c}\right)
\end{aligned}
$$

We choose $\kappa_{0}=C(\tilde{\Delta}, u) / D_{0}^{2}$ so that we have

$$
\operatorname{Tr} E_{\Lambda}(\Delta) E_{0}^{\Lambda}(\tilde{\Delta}) \leq \frac{2}{C(\tilde{\Delta}, u)}\left|\operatorname{Tr} E_{\Lambda}(\Delta) \tilde{V}_{\Lambda} E_{0}^{\Lambda}(\tilde{\Delta})\right|+\frac{D_{0}^{2}}{C(\tilde{\Delta}, u)^{2}} \operatorname{Tr} E_{\Lambda}(\Delta) E_{0}^{\Lambda}\left(\tilde{\Delta}^{c}\right) .
$$


As for the first term on the right in (4.54), we use Hölder's inequality and write

$$
\begin{aligned}
& \left|\operatorname{Tr}_{\Lambda}(\Delta) \tilde{V}_{\Lambda} E_{0}^{\Lambda}(\tilde{\Delta})\right| \\
& \quad \leq\left\|E_{0}^{\Lambda}(\tilde{\Delta}) E_{\Lambda}(\Delta)\right\|_{2}\left\|E_{\Lambda}(\Delta) \tilde{V}_{\Lambda} E_{0}^{\Lambda}(\tilde{\Delta})\right\|_{2} \\
& \quad \leq \frac{1}{2 \sigma}\left\|E_{0}^{\Lambda}(\tilde{\Delta}) E_{\Lambda}(\Delta)\right\|_{2}^{2}+\frac{\sigma}{2}\left\|E_{\Lambda}(\Delta) \tilde{V}_{\Lambda} E_{0}^{\Lambda}(\tilde{\Delta})\right\|_{2}^{2} \\
& \quad \leq \frac{1}{2 \sigma} \operatorname{Tr} E_{0}^{\Lambda}(\tilde{\Delta}) E_{\Lambda}(\Delta)+\frac{\sigma}{2} \operatorname{Tr} E_{0}^{\Lambda}(\tilde{\Delta}) \tilde{V}_{\Lambda} E_{\Lambda}(\Delta) \tilde{V}_{\Lambda} E_{0}^{\Lambda}(\tilde{\Delta}),
\end{aligned}
$$

for any constant $\sigma>0$. In light of the coefficient in (4.54), we choose $\sigma=2 / C(\tilde{\Delta}, u)$ and obtain from (4.54) and (4.55),

$$
\begin{aligned}
\operatorname{Tr} E_{\Lambda}(\Delta) E_{0}^{\Lambda}(\tilde{\Delta}) \leq & \frac{4}{C(\tilde{\Delta}, u)^{2}} \operatorname{Tr} E_{0}^{\Lambda}(\tilde{\Delta}) \tilde{V}_{\Lambda} E_{\Lambda}(\Delta) \tilde{V}_{\Lambda} E_{0}^{\Lambda}(\tilde{\Delta}) \\
& +\frac{2 D_{0}^{2}}{C(\tilde{\Delta}, u)^{2}} \operatorname{Tr} E_{\Lambda}(\Delta) E_{0}^{\Lambda}\left(\tilde{\Delta}^{c}\right) .
\end{aligned}
$$

The second term on the right in (4.56) is bounded above as in (4.47) and (4.49). 4. We estimate the first term on the right in the last line of (4.56). Let $f_{\Delta} \in$ $C_{0}^{\infty}(\mathbb{R})$ be a smooth, compactly-supported, nonnegative function $0 \leq f \leq 1$, with $f_{\Delta} \chi_{\Delta}=\chi_{\Delta}$, where $\chi_{\Delta}$ is the characteristic function on $\Delta$. Note that we can take $\mid$ supp $f \mid \sim 1$ so that the derivatives of $f$ are order one. By positivity, we have the bound

$$
\begin{aligned}
& \operatorname{Tr} E_{0}^{\Lambda}(\tilde{\Delta}) \tilde{V}_{\Lambda} E_{\Lambda}(\Delta) \tilde{V}_{\Lambda} E_{0}^{\Lambda}(\tilde{\Delta}) \\
& \quad=\operatorname{Tr} E_{\Lambda}(\Delta) \tilde{V}_{\Lambda} E_{0}^{\Lambda}(\tilde{\Delta}) \tilde{V}_{\Lambda} E_{\Lambda}(\Delta) \\
& \quad \leq \operatorname{Tr} E_{\Lambda}(\Delta) \tilde{V}_{\Lambda} f_{\Delta}\left(H_{0}^{\Lambda}\right) \tilde{V}_{\Lambda} E_{\Lambda}(\Delta) .
\end{aligned}
$$

Recall that $\chi_{j}$ is a compactly-supported function so that $u_{j} \chi_{j}=u_{j}$. Upon expanding the potential $\tilde{V}_{\Lambda}$, the term on the right in (4.57) is

$$
\sum_{j, k \in \tilde{\Lambda}} \operatorname{Tr} u_{k} E_{\Lambda}(\Delta) u_{j} \cdot \chi_{j} f_{\Delta}\left(H_{0}^{\Lambda}\right) \chi_{k}
$$

The operator $\chi_{j} f_{\Delta}\left(H_{0}^{\Lambda}\right) \chi_{k}$ is a nonrandom, trace class operator. As with the operator $K_{i j}$ in (4.44), it admits a canonical representation

$$
\chi_{j} f_{\Delta}\left(H_{0}^{\Lambda}\right) \chi_{k}=\sum_{l} \lambda_{l}^{(j k)}\left|\phi_{l}^{(j k)}\right\rangle\left\langle\psi_{l}^{(j k)}\right|
$$

for orthonormal functions $\phi_{l}^{(j k)}$ and $\psi_{l}^{(j k)}$. This operator also satisfies a decay estimate of the type

$$
\left\|\chi_{j} f_{\Delta}\left(H_{0}^{\Lambda}\right) \chi_{k}\right\|_{1} \leq C_{N}(f)\left(1+\|k-j\|^{2}\right)^{-N},
$$

for any $N \in \mathbb{I}$ and a finite positive constant depending on $\left\|f^{(j)}\right\|$ independent of $|\Delta|$. This can be proved using the Helffer-Sjöstrand formula, see, for example, [64]. 
Expanding the trace in (4.58) as in (4.47), we can bound (4.58) from above by

$$
\begin{aligned}
\operatorname{Tr} E_{0}^{\Lambda}(\tilde{\Delta}) \tilde{V}_{\Lambda} E_{\Lambda}(\Delta) \tilde{V}_{\Lambda} E_{0}^{\Lambda}(\tilde{\Delta})= & \sum_{l} \sum_{j, k \in \tilde{\Lambda}} \lambda_{l}^{(j k)}\left\langle\psi_{l}^{(j k)}, u_{j} E_{\Lambda}(\Delta) u_{k} \phi_{l}^{(j k)}\right\rangle \\
\leq & \sum_{l} \sum_{j, k \in \tilde{\Lambda}} \lambda_{l}^{(j k)}\left\{\left\langle\psi_{l}^{(j k)}, u_{j} E_{\Lambda}(\Delta) u_{j} \psi_{l}^{(j k)}\right\rangle\right. \\
& \left.+\left\langle\phi_{l}^{(j k)}, u_{k} E_{\Lambda}(\Delta) u_{k} \phi_{l}^{(j k)}\right\rangle\right\} .
\end{aligned}
$$

It is now clear that each matrix element can be estimated as in part 3 applying (4.37). This completes the proof.

\section{Resolvent Estimates and Multiscale Analysis}

5.1. Introduction. In chapter 3 we derived a necessary condition for the absence of singular and absolutely continuous spectrum for a one-parameter perturbation of an operator. We showed that the models discussed in chapter 1 fit into this framework if we consider the variation of one-coupling constant while holding the others fixed. In order to apply this theory, we must show that our family of Hamiltonians satisfy the condition (A2) of section 3.4 for Lebesgue almost-every energy in an interval near the band edges. Since the perturbed and unperturbed Hamiltonians have structurally the same form (they differ only in the realization of the perturbation), we must verify that

$$
\sup _{\epsilon \neq 0}\|R(E+i \epsilon) u\| \leq C(E),
$$

for some finite constant and for almost-every energy in an interval near $\tilde{B}_{-}$and $\tilde{B}_{+}$.

This is an estimate on the infinite-volume Hamiltonian which we expect to have dense pure point spectrum in the energy interval we are considering. Thus, we cannot expect such an estimate to hold at every energy. What saves the situation is the fact that we are dealing with a random family of operators. Since the probability that any fixed energy $E$ is an eigenvalue of $H_{\omega}$ is zero, we can expect that a bound like (5.1) might hold with probability one.

How can we expect to prove such an infinite-volume estimate? Following the notion of thermodynamic limit in statistical mechanics, Fröhlich and Spencer [56] proved an almost-sure fixed energy resolvent bound for the multidimensional lattice Anderson model through an iterative process starting with estimates on finitevolume Hamiltonians. This technique is called multiscale analysis (MSA). The work of Fröhlich and Spencer opened the way to proving localization for multidimensional models. The technique was refined in the work of von Dreifus and Klein [131], Spencer [120], and extended to continuous models in Holden and Martinelli [101], Combes and Hislop [25], Barbaroux, Combes, and Hislop [8], and Kirsch, Stollmann, and Stolz [86].

In this chapter, we provide a general framework for the fixed energy multiscale analysis. We show that infinite-volume estimates such as (5.1) can be derived from two basic estimates on the finite-volume Hamiltonians. These two estimates are the Wegner estimate, proved in chapter 4 , and the initial length scale estimate, referred to as $[H 1]\left(\ell_{0}, \gamma_{0}\right)$. The estimate $[H 1]\left(\ell_{0}, \gamma_{0}\right)$ on the finite-volume Hamiltonians is proved for additive perturbations in chapter 6 . 
We also show how to improve these estimates in order to obtain exponential localization. The details of the inductive step in the multiscale analysis are presented in chapter 6. Finally, we must show how to turn these fixed-energy estimates for the resolvent with respect to one coupling coefficient into an almost sure estimate with respect to the realizations of the potential. We present this probabilistic argument in the last section of this chapter.

5.2. Some Probability Theory: The Borel-Cantelli Lemmas. We will use repeatedly the two Borel-Cantelli Lemmas that we recall here. Let $\left\{A_{n}\right\}$ be a family of subsets of a given set $X$. We define two subsets of $X, \lim \sup A_{n}$ and the $\liminf A_{n}$, by

$$
\liminf _{n} A_{n} \equiv \bigcup_{k=1}^{\infty}\left(\bigcap_{k \geq j} A_{j}\right)
$$

and

$$
\underset{n}{\lim \sup } A_{n} \equiv \bigcap_{k=1}^{\infty}\left(\bigcup_{k \geq j} A_{j}\right) .
$$

Let us recall that $x \in \liminf _{n} A_{n}$ if and only if $x$ belongs to all but finitely many of the sets $A_{n}$. Similarly, $x \in \limsup _{n} A_{n}$ if and only if $x$ belongs infinitely many of the sets $A_{n}$. As subsets of $X$, it is clear that $\liminf { }_{n} A_{n} \subset \lim \sup _{n} A_{n}$.

TheOREM 5.1. (The First Borel-Cantelli Lemma) Let $\left\{A_{n} \in \mathcal{F}\right\}$ be a countable family of measurable subsets of $\Omega$ such that $\sum_{n} \mathbb{P}\left(A_{n}\right)<\infty$. Then, the measure of the set of points that lie in infinitely-many of the sets $A_{n}$ (that is, which occur infinitely-often) is zero. That is, $\mathbb{P}\left\{\limsup _{n} A_{n}\right\}=0$.

TheOrem 5.2. (The Second Borel-Cantelli Lemma) Let $\left\{A_{n} \in \mathcal{F}\right\}$ be a countable family of measurable subsets of $\Omega$ which are independent and for which $\sum_{n} \mathbb{P}\left(A_{n}\right)=$ $\infty$. Then, the measure of the points that lie in infinitely-many of the sets $A_{n}$ (that is, which occur infinitely-often) is one. That is, $\mathbb{P}\left\{\lim \sup _{n} A_{n}\right\}=1$.

5.3. The Geometric Resolvent Equation. The basic idea which makes the multiscale analysis practical for continuous models (see [101] for a presentation using boundary conditions) is to use geometric methods for analysis of resolvents which have been used extensively in semiclassical analysis (see, for example, [75]). We review the notation that we have used in previous chapters. Let $\Lambda_{l}$ be a cube of side $l$ centered at the origin

$$
\Lambda_{l}=\left\{x \in \mathbb{R}^{d}|| x_{i} \mid<l / 2, i=1, \ldots, d\right\} .
$$

We fix some $\delta>0$. In practice, this $\delta$ is determined by diam $(\operatorname{supp} u)$. Let $\Lambda_{\ell, \delta} \subset \Lambda_{l}$ be the subcube defined by

$$
\Lambda_{\ell, \delta} \equiv\left\{x \in \Lambda_{l} \mid \operatorname{dist}\left(x, \partial \Lambda_{l}\right)>\delta\right\} .
$$

Note that $\delta$ is independent of $l$. Let $\Lambda_{l}(x)$ be a cube centered at $x \in \mathbb{R}^{d}$. We need smoothed characteristic functions $\chi_{l}$ such that $\chi_{l} \geq 0, \chi_{l} \mid \Lambda_{\ell, \delta}=1$, and $\operatorname{supp}\left(\nabla \chi_{l}\right) \subset \Lambda_{l} \backslash \Lambda_{\ell, \delta}$. When working with length scales $l_{k}$, we write $\chi_{k}$ and $\Lambda_{k}(x)$, for notational convenience. We also define $\Lambda_{\delta}$ to be the subset of $\Lambda$ defined as in (5.5). 
We treat the additive case in this chapter. We consider a random family $H_{\omega}=$ $H_{0}+V_{\omega}$ of self-adjoint operators with a common dense domain $D_{0} \subset L^{2}\left(\mathbb{R}^{d}\right)$. For any regular region $\Lambda \subset \mathbb{R}^{d}$, we define $H_{\Lambda}$ to be the local Hamiltonian associated with $\Lambda$ by either

$$
H_{\Lambda}=H_{0}+V_{\Lambda}, \text { on } L^{2}\left(\mathbb{R}^{d}\right)
$$

where

$$
V_{\Lambda} \equiv V \mid \Lambda
$$

or by

$$
H_{\Lambda}=\left(H_{0}+V_{\omega}\right) \mid \Lambda, \text { on } L^{2}(\Lambda),
$$

with self-adjoint boundary conditions on $\partial \Lambda$. It is often convenient to use one form or the other of the local Hamiltonian. The MSA is the same regardless of the form used except that the equations hold on $L^{2}\left(\mathbb{R}^{d}\right)$ in the first case, or $L^{2}(\Lambda)$ in the second case. We will write $\mathcal{H}$ for either space.

Let $R_{\Lambda}(z)=\left(H_{\Lambda}-z\right)^{-1}$ be the resolvent of $H_{\Lambda}$ on $\mathcal{H}$. For two regions $\Lambda \subset$ $\Lambda^{\prime} \subset \mathbb{R}^{d}$, we can compare the resolvents $R_{\Lambda}(z)$ and $R_{\Lambda^{\prime}}(z)$ associated with the local Hamiltonians as follows. Let $W_{\Lambda}$ be the first-order differential operator defined by

$$
W_{\Lambda}=\left[H_{0}, \chi_{\Lambda}\right] .
$$

We recall that $\operatorname{supp}\left(\nabla \chi_{\Lambda}\right) \subset \Lambda \backslash \Lambda_{\delta}$ so that $W_{\Lambda}$ is localized near $\partial \Lambda$. To compare the two resolvents, $R_{\Lambda}(z)$ and $R_{\Lambda^{\prime}}(z)$, we write

$$
\chi_{\Lambda}\left(H_{\Lambda^{\prime}}-z\right)=\left(H_{\Lambda}-z\right) \chi_{\Lambda}-W_{\Lambda},
$$

from which it follows that

$$
\chi_{\Lambda} R_{\Lambda^{\prime}}(z)=R_{\Lambda}(z) \chi_{\Lambda}+R_{\Lambda}(z) W\left(\chi_{\Lambda}\right) R_{\Lambda^{\prime}}(z),
$$

acting as an operator on $L^{2}\left(\mathbb{R}^{d}\right)$ or on $L^{2}\left(\Lambda^{\prime}\right)$, where $\chi_{\Lambda}$ serves as the natural injection. By taking the adjoint of (5.11) and replacing $\bar{z}$ by $z$, we can also write this equation as

$$
R_{\Lambda^{\prime}}(z) \chi_{\Lambda}=\chi_{\Lambda} R_{\Lambda}(z)-R_{\Lambda^{\prime}}(z) W\left(\chi_{\Lambda}\right) R_{\Lambda}(z) .
$$

We used the fact that $V_{\Lambda^{\prime}} \mid \Lambda=V_{\Lambda}$. This last relation $V_{\Lambda^{\prime}} \mid \Lambda=V_{\Lambda}$ does not always hold, especially if the support of the single-site potential extends beyond the unit cube $\Lambda_{1}(0)$. The difference, however, can be easily incorporated into the MSA. We refer to either of the geometric resolvent eqautions, (5.11) or (5.12), as the GRE.

5.4. The ELF Theorem. The name of this theorem was inspired by the French oil company ELF, since it is what makes the MSA work. We also name the ingredients $\epsilon$, length-scale $\ell$, and $f$. This is the basic theorem that allows us to control the iteration of resolvent estimates for finite-volume Hamiltonians over several increasing length scales. We need the following:

(i) $\left\{\epsilon_{n}\right\}$, a monotone decreasing sequence of positive numbers with $\lim _{n \rightarrow \infty} \epsilon_{n}=0$

(ii) $\left\{l_{k}\right\}$, a monotone increasing length scale: $l_{k} \uparrow \infty$;

(iii) $f>0$, is a non-decreasing function with $(1 / f) \in L_{\text {loc }}^{2}(\mathbb{R})$. 
For example, we can take $f(s)=\langle s\rangle^{\alpha}$, for any $\alpha<1 / 2$.

We need two basic hypotheses on the local Hamiltonians $H_{k} \equiv H_{\Lambda_{\ell_{k}}}$, where $\ell_{k}$ is a length scale as in (ii) above, and their resolvents $R_{k}(z) \equiv\left(H_{k}-z\right)^{-1}$ :

[H1]. For each $k \in \mathbb{N}$, there exists a probability $\tilde{P}_{k}$, with $\left(1-\tilde{P}_{k}\right) \in \ell^{1}(\mathbb{I N})$, such that

$$
\mathbb{P}\left\{\sup _{\epsilon>0}\left\|W\left(\chi_{k}\right) R_{k}(\epsilon) \chi_{k-1}\right\| \leq \epsilon_{k+1}^{2}\right\} \geq \tilde{P}_{k}
$$

Note that this requires estimates over multiple length scales, and is not practical from a computational point of view. We will improve this below. The second hypothesis is a Wegner estimate on each length scale:

[H2]. For some sequences $\left\{l_{k}\right\}$ and $\left\{\epsilon_{k}\right\}$, as in [H1], we have $\forall k \in \mathbb{N}$,

$$
P_{k} \equiv \mathbb{P}\left\{\operatorname{dist}\left(\sigma\left(H_{k}\right), E\right)<f\left(\epsilon_{k}\right)\right\} \in \ell^{1}(I N) .
$$

Given these two hypotheses, the basic result is

THEOREM 5.3. Let $\left\{l_{k}\right\},\left\{\epsilon_{k}\right\}$, and $f$ be as above and suppose $c$ is a function of compact support. If [H1] and [H2] hold, then for IP-almost every $\omega$ there exists a constant $0<d_{\omega}<\infty$ such that

$$
\sup _{\epsilon>0}\left\|(H-E-i \epsilon)^{-1} c\right\| \leq d_{\omega}\|c\|_{\infty} .
$$

Proof. Since $c$ has compact support, there is a $k_{0}$ so that for all $k>k_{0}$, we have $\chi_{k} c=c$. We choose $k$ large enough so $\chi_{k-1} c=c$. Let $R\left(\epsilon_{k}\right) \equiv\left(H-E-i \epsilon_{k}\right)^{-1}$. Applying the geometric resolvent equation twice, we obtain,

$$
\begin{aligned}
R\left(\epsilon_{k+1}\right) R\left(\epsilon_{k}\right) c= & R\left(\epsilon_{k+1}\right)\left\{\chi_{k} R_{k}\left(\epsilon_{k}\right)+R\left(\epsilon_{k}\right) W_{k} R_{k}\left(\epsilon_{k}\right)\right\} c \\
= & \chi_{k} R_{k}\left(\epsilon_{k+1}\right) R_{k}\left(\epsilon_{k}\right) c+R\left(\epsilon_{k+1}\right) W_{k} R_{k}\left(\epsilon_{k+1}\right) R_{k}\left(\epsilon_{k}\right) c \\
& +R\left(\epsilon_{k+1}\right) R\left(\epsilon_{k}\right) W_{k} R_{k}\left(\epsilon_{k}\right) c \\
= & I+I I+I I I .
\end{aligned}
$$

By hypothesis [H1],

$$
\|I I I\| \leq\|c\|_{\infty} \epsilon_{k+1}^{2} \cdot\left(\frac{1}{\epsilon_{k+1}}\right)\left(\frac{1}{\epsilon_{k}}\right) \leq\|c\|_{\infty},
$$

with probability $\geq \tilde{P}_{k}$. As for $I I$, we use the first resolvent formula for $R_{k}$ and obtain,

$$
\begin{aligned}
& \left\|R\left(\epsilon_{k+1}\right) W_{k}\left[R_{k}\left(\epsilon_{k+1}\right)-R_{k}\left(\epsilon_{k}\right)\right]\left(\epsilon_{k+1}-\epsilon_{k}\right)^{-1} c\right\| \\
& \leq \frac{1}{\epsilon_{k+1}} \cdot \frac{1}{\left(\epsilon_{k}-\epsilon_{k+1}\right)} \cdot\left(\left\|W_{k} R_{k}\left(\epsilon_{k+1}\right) c\right\|+\left\|W_{k} R_{k}\left(\epsilon_{k}\right) c\right\|\right) .
\end{aligned}
$$

By choosing a subsequence, if necessary, we assume that $\epsilon_{k}>2 \epsilon_{k+1}$. Then, the bound in (5.18) is less than

$$
2 \cdot \frac{1}{\epsilon_{k+1}^{2}} \cdot \epsilon_{k+1}^{2} \cdot\|c\|_{\infty} \leq 2\|c\|_{\infty}
$$

with a probability $\geq \tilde{P}_{k}$. As for $I,[\mathrm{H} 2]$ implies that

$$
\|I\| \leq \frac{1}{f\left(\epsilon_{k+1}\right)} \frac{1}{f\left(\epsilon_{k}\right)}\|c\|_{\infty} \leq \frac{1}{f\left(\epsilon_{k}\right)^{2}}\|c\|_{\infty},
$$


PETER D. HISLOP

with a probability $\geq 1-P_{k}$. Hence, the event

$$
B_{k} \equiv\left\{\omega \in \Omega \mid\left\|R\left(\epsilon_{k+1}\right) R\left(\epsilon_{k}\right) c\right\| \leq \frac{2\|c\|_{\infty}}{f\left(\epsilon_{k}\right)^{2}}\right\},
$$

occurs with probability

$$
\mathbb{P}\left(B_{k}\right) \geq \tilde{P}_{k}-P_{k}
$$

or

$$
\mathbb{P}\left(B_{k}^{c}\right) \leq 1-\tilde{P}_{k}+P_{k}
$$

But, by our assumptions,

$$
\sum_{k} \mathbb{P}\left(B_{k}^{c}\right) \leq \sum_{k}\left(1-\tilde{P}_{k}\right)+\sum_{k} P_{k}<\infty .
$$

By the first Borel Cantelli lemma 5.1, it follows that $\mathbb{P}\left\{\limsup _{k} B_{k}^{c}\right\}=0$. As $\left(\limsup B_{k}^{c}\right)^{c}=\liminf B_{k}$, we have that $\mathbb{P}\left(\liminf B_{k}\right)=1$. So for any $\omega \in$ $\liminf { }_{k} B_{k}$, there exists a $k_{0}(\omega)$, so that for all $k>k_{0}(\omega)$, the event on the right in (5.21) occurs. Consequently, for $k>k_{0}(\omega)$, we write

$$
\begin{aligned}
R\left(\epsilon_{k}\right) & =R\left(\epsilon_{k_{0}}\right)+\sum_{l=k_{0}}^{k-1}\left(R\left(\epsilon_{l+1}\right)-R\left(\epsilon_{l}\right)\right) \\
& =R\left(\epsilon_{k_{0}}\right)+\sum_{l=k_{0}}^{k-1}\left(\epsilon_{l+1}-\epsilon_{l}\right) R\left(\epsilon_{l+1}\right) R\left(\epsilon_{l}\right) .
\end{aligned}
$$

Since $\omega \in B_{l}$, we use the estimate on the right in (5.21), and obtain

$$
\left\|R\left(\epsilon_{k}\right) c\right\| \leq\left\|R\left(\epsilon_{k_{0}}\right) c\right\|+\sum_{l=k_{0}}^{k-1}\left(2\|c\|_{\infty} \frac{\left(\epsilon_{l}-\epsilon_{l+1}\right)}{f\left(\epsilon_{l}\right)^{2}}\right) .
$$

The sum is finite and bounded by a constant due to the fact that $(1 / f)^{2} \in L^{1}(\mathbb{R})$. As a consequence, for each $\omega \in \liminf B_{k}$, a set of full measure, there is a finite constant $C(c, \omega)>0$ so that

$$
\limsup _{k \rightarrow \infty}\left\|R\left(\epsilon_{k}\right) c\right\| \leq C(c, \omega)<\infty,
$$

with probability one.

5.5. Reduction to an Initial Length Scale Estimate. We next want to show how to simplify [H1] by reducing it to an initial estimate at one length scale. We have two new hypotheses modifying [H1]-[H2].

$[\mathrm{H} 1]\left(\gamma_{0}, l_{0}\right)$. For some $\gamma_{0}>0$ and length $l_{0}>>1$, such that $\gamma_{0} \ell_{0}>>1$, there exists an exponent $\xi>2 d$ such that

$$
\mathbb{P}\left\{\sup _{\epsilon>0}\left\|W\left(\chi_{l_{0}}\right) R_{\Lambda_{l_{0}}}(E+i \epsilon) \chi_{l_{0} / 3}\right\| \leq e^{-\gamma_{0} \ell_{0}}\right\} \geq 1-l_{0}^{-\xi} .
$$

The next version of [H2] is satisfied by the models for which a Wegner's estimate can be proved. 
[W] There exists constants $0<C_{W}<\infty$, exponent $1 \leq \tau<$ infty, and $0<q \leq 1$, such that for all sufficiently large $\Lambda$, and all $\eta>0$,

$$
\mathbb{P}\left\{\operatorname{dist}\left(\sigma\left(H_{\Lambda}\right), E\right)<\eta\right\} \leq C_{W}|\Lambda|^{\tau} \eta^{q} .
$$

The main technique result, which requires the multiscale analysis, consists in proving

$$
[\mathrm{H} 1]\left(\gamma_{0}, l_{0}\right)+[\mathrm{W}] \Rightarrow[\mathrm{H} 1]
$$

This is given in the following lemma, whose proof we give in chapter 8 .

Lemma 5.4. Let $\gamma_{0}>0$ and assume $[W]$. There exists a minimum length scale $l^{*}=l^{*}\left(\gamma_{0}, C_{W}, \tau, q, d\right)$, such that if $[H 1]\left(\gamma_{0}, l_{0}\right)$ holds for $l_{0}>l^{*}$, then for length scales $l_{k} \equiv l_{0}^{(3 / 2)^{k}}, \xi>2 d, \exists 0<\kappa<\infty$ such that for each $k$

$$
P\left\{\left\|W_{l_{k}} R_{l_{k}}(E+i \epsilon) \chi_{l_{k} / 3}\right\| \leq e^{-\kappa \gamma_{0} l_{k}}\right\} \geq 1-l_{k}^{-\xi} .
$$

A consequence of this lemma is an improvement in the hypotheses of Theorem 5.3 .

THEOREM 5.5. Let $\gamma_{0}>0$ and assume $[W]$. There exists a minimum length scale $l^{*}=l^{*}\left(\gamma_{0}, C_{W}, \tau, q, d\right)$, such that if $[H 1]\left(\gamma_{0}, \ell_{0}\right)$ holds for $\ell_{0}>\ell^{*}$, and if $c$ is any function of compact support, then for almost every $\omega$, there exists a finite constant $d_{\omega}(c)<\infty$ such that

$$
\sup _{\epsilon>0}\left\|(H-E-i \epsilon)^{-1} c\right\| \leq d_{\omega} \delta(c) .
$$

Proof. From Lemma 5.4, we have [H1] in a strong form with:

$$
\begin{aligned}
& \epsilon_{k+1}=\left(l_{k}\right)^{-\left(3+\delta_{1}\right) \tau d}, 0<\delta_{1}<<1 ; \\
& l_{k}=l_{0}^{(3 / 2)^{k}} ; \\
& \tilde{P}_{k}=1-l_{k}^{-\xi}, \xi>2 d .
\end{aligned}
$$

We apply Theorem 5.1 with $f(\epsilon)=\epsilon^{1 / 2-\delta}, 0<\delta<<\delta_{1}$. Then, we see that $1-\tilde{P}_{k} \in l^{1}, f^{-1} \in L_{\text {loc }}^{2}$ and

$$
\left|\Lambda_{k}\right|^{\tau} f\left(\epsilon_{k}\right)=l_{k}^{-\sigma}
$$

for some $\sigma>0$. This verifies the assumptions of the theorem.

5.6. Exponential Decay Estimates. We now obtain exponential decay estimates on the infinite-volume resolvents with good probability. We observe that Theorem 5.5 suffices to prove localization by condition (A2), Theorem 3.4, and the results of the next section. If we want exponential decay of the eigenfunctions, we need stronger estimates on the Green's function. In the case that $\operatorname{supp} u$ is not compact, we can only get polynomial decay estimates. 
THEOREM 5.6. Let $\chi_{x}$ be the characteristic function of a unit cube centered at $x$. Then, under the assumptions of Theorem 5.5, we have

(1) If c has compact support, then with probability one there exists a constant $0<$ $d_{\omega}<\infty$ such that for all large $\|x\|$,

$$
\sup _{\epsilon>0}\left\|\chi_{x} R(E+i \epsilon) c\right\| \leq d_{\omega}\|c\|_{\infty} e^{-\frac{1}{6 \sqrt{d}} \gamma_{0}\|x\|} .
$$

(2) If c satisfies $|c(x)|<\delta(c)(1+\|x\|)^{-\beta}$, with $\beta>9 d \tau / 2$, then with probability one there exists a constant $0<d_{\omega}<\infty$ such that for $\|x\|$ large,

$$
\sup _{\epsilon>0}\left\|\chi_{x} R(E+i \epsilon) c\right\| \leq d_{\omega} \delta(c)\|x\|^{-\beta / 2}
$$

Proof. We take $\|x\|$ large so $3 l_{k-1}<|x|<3 l_{k}$ for some $k$. Applying the GRE (5.11) to $R$ and $R_{8 l_{k}}$, we obtain

$$
\chi_{x} R c=\chi_{x} R_{8 l_{k}} \chi_{8 l_{k}} c+\chi_{x} R_{8 l_{k}} W_{8 l_{k}} R c .
$$

Let $c_{k} \equiv c \mid \Lambda_{l_{k-1}}$. We estimate (5.33) by

$$
\left\|\chi_{x} R c\right\| \leq\left\|\chi_{x} R_{8 l_{k}} c_{k}\right\|+\left\|\chi_{x} R_{8 l_{k}} \chi_{8 l_{k}}\left(c-c_{k}\right)\right\|+c_{0}\left\|\chi_{x} R_{8 l_{k}} W_{8 l_{k}}\right\|,
$$

where we used Theorem 5.3 to bound $\|R c\|$ with probability one. We now bound the first and third terms using a variation of Lemma 5.4. When $\operatorname{supp} c$ is non-compact one must use the decay of $c$ to estimate the second term.

Theorem 5.6 reduces the proof of exponential localization to the verification of $[\mathrm{H} 1]\left(\gamma_{0}, l_{0}\right)$ and $[\mathrm{W}]$. The proof of $[\mathrm{W}]$ is given in chapter 4 . For the additive models of chapter 1 , the proof of $[\mathrm{H} 1]\left(\gamma_{0}, l_{0}\right)$ is presented in chapter 6 .

5.7. Probabilistic Estimates for Localization. Let us fix a closed energy interval $I$ for which we can verify conditions $[H 1]\left(\gamma_{0}, \ell_{0}\right)$ and $[\mathrm{W}]$ for the finitevolume Hamiltonians. According to Theorem 5.3, we can then verify the key hypothesis of (A2) on the perturbation of one-parameter families at each energy $E \in I$. Recall that we consider fixing all but one random variable, say $\lambda_{0}$, and study the spectrum of the resulting one-parameter family of operators. The main result of Theorem 3.4 states that under the condition (5.28) on the unperturbed Hamiltonian, the spectrum of the one-parameter family in the interval $I$ is dense pure point.

What we have proved is the following: for $\mathbb{P}$-almost every configuration $\omega$, there is a set $J_{\omega}$ of values of the random variable $\lambda_{0}$ for which the resulting oneparameter family of operators $H_{\omega_{0}, \omega^{\perp}}$ has pure point spectrum in the interval $I$. The problem is that the set of $\lambda_{0}$ values $J_{\omega}$ depends on the initial configuration $\omega$ chosen. Another way to think of this is as follows. Consider the configurations $\hat{\omega}$ having the property that $\lambda_{0}(\hat{\omega})=0$. There is a set of values $\lambda_{0}$ for which the configurations $\omega=\left(\hat{\omega}, \lambda_{0}\right)$ for which (5.28) holds at almost-every energy in $I$. We will show that configurations of this form have full measure. This will imply the result. Hence, we need to turn this result into a result for almost-every configuration $\omega$.

In order to do this, we introduce a device of extending the probability space $(\Omega, \mathbb{P})$ in the following manner. Let us define subsets $\Omega_{0} \subset \Omega$ and $\Omega_{1} \subset \Omega$ by

$$
\Omega_{0} \equiv\left\{\omega \mid \sup _{\epsilon>0}\left\|(H-E-i \epsilon)^{-1} c\right\| \leq \delta(c) C_{\omega, E}<\infty \forall E \in I\right\}
$$


and for any function $c$ as in Theorem 5.6, and

$$
\Omega_{1} \equiv\left\{\omega \mid H_{\omega} \text { is pure point in } I \text { with finitely degenerate eigenvalues }\right\} .
$$

The weak measurability of the spectral projectors show that the set $\Omega_{1}$ is measurable. Our goal is to show that $\mathbb{P}\left(\Omega_{1}\right)=1$. Let $\omega \in \Omega_{0}$ and consider the variation of the random variable $\lambda_{0}$. As in chapter 3 , we write

$$
H_{\lambda, \omega}=H_{\omega}+\lambda u \text {. }
$$

By Theorem 3.2, there exists a set $S_{\omega} \subset \operatorname{supp} g$, such that $\int_{S_{\omega}} g(\lambda) d \lambda=1$, for which the following holds:

$$
\begin{aligned}
\lambda \in S_{\omega} \rightarrow & \left\{\tilde{H}_{\lambda, \omega} \text { has pure point spectrum in } I\right. \\
& \text { with finite multiplicities }\} .
\end{aligned}
$$

Here we write $\tilde{H}_{\lambda, \omega}$ for the restriction of the operator in (5.37) to the cyclic subspace generated by the action of $f(H)$ on the set $\left\{u^{1 / 2} \phi \mid \phi \in L^{2}\left(\mathbb{R}^{d}\right)\right\}$. In our models, this cyclic subspace is the entire Hilbert spaces, so we will drop the tilde from the notation. We define a third subset of $\Omega$ by

$$
\Omega_{2} \equiv\left\{\left(\omega+\lambda \delta_{0 i}\right) \mid \omega \in \Omega_{0} \text { and } \lambda \in S_{\omega}\right\} .
$$

The notation $\omega+\lambda \delta_{0 i}$ means $\lambda_{j}\left(\omega+\lambda \delta_{0 i}\right)=\lambda_{j}(\omega)$, for $j \neq 0$, and $\lambda_{0}\left(\omega+\lambda \delta_{0 i}\right)=$ $\lambda_{0}(\omega)+\lambda$. Note that $\Omega_{2} \subset \Omega_{1}$.

We now consider the quotient of each of the subspaces $\Omega_{i}$ by $\mathbb{R}$ along the zero site. We denote by $\hat{\omega}$ those configurations in $\Omega$ for which $\lambda_{0}(\hat{\omega})=0$. We define the sets

$$
\tilde{\Omega}_{i} \equiv\left\{\hat{\omega} \mid \exists \mu \in \mathbb{R} \text { so that } \hat{\omega}+\mu \delta_{0 i} \in \Omega_{i}\right\} .
$$

We note an important identity: $\tilde{\Omega}_{0}=\Omega_{2} / \mathbb{R} \subset \tilde{\Omega}_{1}$. Given an element $\tilde{\omega} \in \tilde{\Omega}_{i}$, we define a section $S\left(\tilde{\omega} \mid \Omega_{i}\right)$ by

$$
S\left(\tilde{\omega} \mid \Omega_{i}\right) \equiv\left\{\lambda \in \mathbb{R} \mid(\hat{\omega}, \lambda) \in \Omega_{i}\right\} .
$$

These subsets of $\mathbb{R}$ are measurable with respect to the measure $g(\lambda) d \lambda$ and have full measure.

We now compute the probability measure of the set $\Omega_{1}$ using Fubini's Theorem. For this, we note that $\Omega_{1}=\tilde{\Omega}_{1} \times S\left(\tilde{\omega} \mid \Omega_{1}\right)$, that $\tilde{\Omega}_{0} \subset \tilde{\Omega}_{1}$, with $\mathbb{P}\left(\tilde{\Omega}_{0}\right)=1$, and that $S\left(\tilde{\omega} \mid \Omega_{1}\right) \subset S\left(\tilde{\omega} \mid \Omega_{2}\right)$. Using these relations, we can write

$$
\begin{aligned}
\int_{\Omega_{1}} d \mathbb{P} & =\int_{\tilde{\Omega}_{1}} d \tilde{\mathbb{P}} \int_{S\left(\tilde{\omega} \mid \Omega_{1}\right)} g(\lambda) d \lambda \\
& \geq \int_{\tilde{\Omega}_{0}} d \tilde{\mathbb{P}} \int_{S\left(\tilde{\omega} \mid \Omega_{2}\right)} g(\lambda) d \lambda \\
& =1 .
\end{aligned}
$$

Given the result on the $H$-cyclicity of the set $\left\{u^{1 / 2} \phi \mid \phi \in L^{2}\left(\mathbb{R}^{d}\right)\right\}$, we have proven that the set $\Omega_{1}$ has full probability measure. 
5.8. Probabilistic Estimates for Exponential Decay. We show how to use the refined decay estimates of Theorem 5.6 to prove exponential decay of the eigenfunctions for those $\omega$ exhibiting localization. The argument is similar to the one presented in subsection 5.7. For the simplicity of presentation, we consider only the case when the single-site potential $u$ has compact support.

Let $\chi_{n}$ be the characteristic function for the unit cube centered at $n \in \mathbb{Z}^{d}$. We define a function $f_{E}(n)$ by

$$
f_{E}(n)=e^{-\gamma_{E}\|n\|} .
$$

We define another set of configurations $\Omega_{0} \subset \Omega$ as follows.

$$
\begin{aligned}
\Omega_{0} \equiv & \left\{\omega \mid \sup _{\epsilon>0}\left\|\chi_{n}(H-E-i \epsilon)^{-1} c\right\| \leq \delta(c) C_{\omega, E} f_{E}(n)\right. \\
& \text { for a. e. } \left.E \in I\left(\delta_{0}\right) \text { and for all } n \text { large }\right\},
\end{aligned}
$$

and for any function $c$ as in Theorem 5.6. It follows from Theorem 5.6 that $\operatorname{IP}\left(\Omega_{0}\right)=$ 1.

We now use the one-parameter theory to show that the eigenfunctions of $H_{\omega}$ decay exponentially. We use the notation $H_{\lambda, \omega}$ as in subsection 5.6. For any $\omega \in \Omega_{0}$, Theorem 3.4 implies that there exists a subset $S(\omega) \subset \mathbb{R}$, of full measure, such that $\lambda \in S(\omega)$ implies that $H_{\lambda, \omega}$ has pure point spectrum in $I\left(\delta_{0}\right)$. For any $E$ which is an eigenvalue of $H_{\lambda, \omega}$ with eigenfunction $\psi_{E}$, it follows from the analysis in chapter 3 that

$$
-s-\lim _{\epsilon \rightarrow 0} \lambda\left(H_{\omega}-E-i \epsilon\right)^{-1} V_{\omega} \psi_{E}=\psi_{E} .
$$

Since $\omega \in \Omega_{0}$, we obtain for any $n \in \mathbb{Z}^{d}$,

$$
\left\|\chi_{n} \psi_{E}\right\| \leq C_{\omega}(E) f_{E}(n),
$$

where we used the normalization $\left\|\psi_{E}\right\|=1$.

Let $P_{\omega}^{E}$ be the spectral projector for $H_{\omega}$ onto the eigenspace for the eigenvalue $E$. We now define another subset $\Omega_{1} \subset \Omega_{0}$ by

$\Omega_{1} \equiv\left\{\omega \in \Omega \mid \sigma\left(H_{\omega}\right) \cap I\left(\delta_{0}\right)\right.$ is pure point and for any $E \in \sigma\left(H_{\omega}\right) \cap I\left(\delta_{0}\right)$,

$$
\text { and for any } \left.\delta>0, \sum_{n}\|n\|^{-1-\delta} f_{E}(n)^{-1}\left\|\chi_{n} P_{\omega}^{E}\right\|<\infty\right\} \text {. }
$$

Since the projector is weakly measurable and bounded, it follows that $\left\|\chi_{n} P_{\omega}^{E}\right\|$ is measurable, which implies that the set $\Omega_{1}$ is measurable.

To prove that $\mathbb{P}\left(\Omega_{1}\right)=1$, we proceed as above. We define another set $\Omega_{2} \subset \Omega$ by

$$
\Omega_{2} \equiv\left\{\omega+\lambda \delta_{0 i} \mid \omega \in \Omega_{0}, \lambda \in S(\omega)\right\},
$$

so that $\Omega_{2} \subset \Omega_{1}$. We now consider the quotient of each of the subspaces $\Omega_{i}$ by $\mathbb{R}$ along the zero site. We denote by $\hat{\omega}$ those configurations in $\Omega$ for which $\lambda_{0}(\hat{\omega})=0$. We define the sets

$$
\tilde{\Omega}_{i} \equiv\left\{\hat{\omega} \mid \exists \mu \in \mathbb{R} \text { so that } \hat{\omega}+\mu \delta_{0 i} \in \Omega_{i}\right\} .
$$

By construction, we have $\tilde{\Omega}_{0}=\tilde{\Omega}_{2} \subset \tilde{\Omega}_{1}$. Given an element $\tilde{\omega} \in \tilde{\Omega}_{i}$, we define a section $S\left(\tilde{\omega} \mid \Omega_{i}\right)$ by

$$
S\left(\tilde{\omega} \mid \Omega_{i}\right) \equiv\left\{\lambda \in \mathbb{R} \mid(\hat{\omega}, \lambda) \in \Omega_{i}\right\}
$$


These subsets of $\mathbb{R}$ are measurable with respect to the measure $g(\lambda) d \lambda$ and have full measure.

We can now compute the probability measure of the set $\Omega_{1}$ using Fubini's Theorem, as above,

$$
\begin{aligned}
\int_{\Omega_{1}} d \mathbb{P} & =\int_{\tilde{\Omega}_{1}} d \tilde{\mathbb{P}} \int_{S\left(\tilde{\omega} \mid \Omega_{1}\right)} g(\lambda) d \lambda \\
& \geq \int_{\tilde{\Omega}_{2}} d \tilde{\mathbb{P}} \int_{S\left(\tilde{\omega} \mid \Omega_{2}\right)} g(\lambda) d \lambda \\
& =1 .
\end{aligned}
$$

Hence, for any $\omega \in \Omega_{2}$, and for any $E \in \sigma\left(H_{\omega}\right) \cap I\left(\delta_{0}\right)$ with eigenfunction $\psi_{E}$, there exists a constant $C_{\omega}(E)$ so that for any $\delta>0$,

$$
\left\|\chi_{n} \psi_{E}\right\| \leq C_{\omega}(E) f(n)\|n\|^{\delta / 2} .
$$

Now let $J_{k}$ be the characteristic function on the set $\mathcal{A}_{k} \equiv\left\{x \in \mathbb{R}^{d} \mid k-1<\|x\|<\right.$ $k, k \in \mathbb{N}\}$. We define a function $F$ by

$$
F(x) \equiv e^{(\gamma-\epsilon)\|x\|} .
$$

We then have

$$
\begin{aligned}
\int\left|F(x) \psi_{E}(x)\right|^{2} & \leq \sum_{k} \int F(x)^{2} J_{k}(x)\left|\psi_{E}(x)\right|^{2} \\
& \leq \sum_{k} C_{\omega}(E) F(k+1)^{2} k^{d+\delta} f(k)^{2},
\end{aligned}
$$

which is finite. We complete the proof of exponential decay by using subsolution estimates, as in [35], to pass from $L^{2}$-bounds to pointwise bounds.

\section{Localization for Families of Random Schrödinger Operators}

6.1. Introduction. We are now in position to prove localization at band-edge energies for random families of Schrödinger operators. In chapter 5, we established two conditions on the local Hamiltonians which will guarantee localization. First, we must establish an initial length-scale estimate for the local Hamiltonians at the energies of interest:

$[\mathbf{H 1}]\left(\gamma_{0}, \ell_{0}\right)$. For some $\gamma_{0}>0$, and for some length scale $\ell_{0}>0$, so that $\gamma_{0} \ell_{0}>>1$, there exists an exponent $\xi>2 d$ such that

$$
\mathbb{P}\left\{\sup _{\epsilon>0}\left\|W\left(\chi_{\ell_{0}}\right) R_{\Lambda_{\ell_{0}}}(E+i \epsilon) \chi_{\ell_{0} / 3}\right\| \leq e^{-\gamma_{0} \ell_{0}}\right\} \geq 1-\ell_{0}^{-\xi} .
$$

Second, we must prove a Wegner estimate at those energies:

[W]. There exist constants $0<C_{W}<\infty, \tau>1$, and $\sigma>0$, so that for all $|\Lambda|$ sufficiently large and for all $\eta>0$,

$$
\mathbb{P}\left\{\operatorname{dist}\left(\sigma\left(H_{\Lambda}\right), E\right)<\eta\right\} \leq C_{W}\left(|\Lambda|^{\tau} \eta+e^{-|\Lambda|^{\sigma}}\right) .
$$

We note that for the Anderson and breather models, the probability estimate in the Wegner estimate is much simpler: one can replace the right side of (6.2) by $C_{W} \eta|\Lambda|$.

We already proved $[W]$ in chapter 4 . We prove condition $[H 1]\left(\gamma_{0}, \ell_{0}\right)$ in this chapter for additive perturbations. We refer the reader to the literature (for example, $[\mathbf{3 3}]$ ) for the case of multiplicative perturbations. Our general approach to 
proving this estimate is to study the dependence of the eigenvalues of the local Hamiltonian $H_{\Lambda}$ on the random variables in $\Lambda$. We will prove that with a good, nonzero probability, the eigenvalues will stay away from a small interval of energies near the band edges $\tilde{B}_{-}$and $\tilde{B}_{+}$. We can then apply the refined version of the Combes-Thomas exponential decay estimate given in [8] in order to establish the exponential decay of the localized resolvent at these energies. We mention that whereas the Wegner estimate holds at all energies in the unperturbed spectral gap $G=\left[B_{-}, B_{+}\right]$, it is the estimate $[H 1]\left(\gamma_{0}, \ell_{0}\right)$ which determines the interval of energies for which we have localization.

6.2. Eigenvalues of the Local Hamiltonians. The goal of this section is to prove the hypothesis $[\mathrm{H} 1]\left(\gamma_{0}, \ell_{0}\right)$ for finite-volume Hamiltonians corresponding to the additively perturbed models introduced in chapter 1 . Let us recall the construction of the finite-volume Hamiltonians. As in previous chapters, we let $\Lambda \subset \mathbb{R}^{d}$ denote a bounded open region, and $\Lambda_{\ell}\left(x_{0}\right) \equiv\left\{x \in \mathbb{R}^{d}|| x_{i}-x_{0, i} \mid<\right.$ $\ell / 2, i=1, \ldots, d\}$. When $x_{0}=0$, we will write $\Lambda_{\ell}$ for simplicity. The potential depending only on the $\omega_{i}$ in a region $\Lambda$ is denoted $V_{\Lambda}=\left(V_{\omega} \mid \Lambda\right)$. The finite-volume Hamiltonians $H_{\Lambda, \omega}$ are defined as $H_{\Lambda, \omega} \equiv H_{0}+V_{\Lambda}$. For notational simplicity, we will omit the $\omega$ when writing $H_{\Lambda}$, when no particular configuration is considered. Since $V_{\Lambda}$ has compact support, it is a relatively compact perturbation of $H_{0}$ and hence $\sigma_{\text {ess }}\left(H_{0}\right)=\sigma_{\text {ess }}\left(H_{\Lambda}\right)$. One of our first tasks is to locate precisely the eigenvalues of $H_{\Lambda}$ in the unperturbed spectral gap $G=\left(B_{-}, B_{+}\right)$with good probability.

The condition $[\mathrm{H} 1]\left(\gamma_{0}, \ell_{0}\right)$ in $(6.1)$ is an estimate on the decay of the on the resolvent of $H_{\Lambda}$, which we write as $R_{\Lambda}(z)=\left(H_{\Lambda}-z\right)^{-1}$, when it exists. We recall that the most general form of $H_{0}$ is $H_{0}=(p-A)^{2}+V_{0}$, where $p \equiv-i \nabla$, and $A$ and $V_{0}$ are periodic vector-valued and scalar functions, respectively. For any $\chi \in C^{2}$, define the first order differential operator $W(\chi)$ by

$$
W(\chi) \equiv\left[H_{0}, \chi\right]=-i(p-A) \cdot \nabla \chi-i \nabla \chi \cdot(p-A) .
$$

This operator is localized on the support of $\nabla \chi$. We choose any $\delta>0$ small, and let $\Lambda_{\ell, \delta} \equiv\left\{x \in \Lambda_{\ell} \mid \operatorname{dist}\left(\partial \Lambda_{\ell}, \chi\right)>\delta\right\}$. We will use $\chi_{\ell}$ to denote a function satisfying $\chi_{\ell} \mid \Lambda_{\ell, \delta}=1, \operatorname{supp} \chi_{\ell} \subset \Lambda_{\ell}$, and $0 \leq \chi_{\ell} \leq 1$. It follows that $\operatorname{supp} \nabla \chi_{\ell} \subset \Lambda_{\ell} \backslash \Lambda_{\ell, \delta}$, and $W\left(\chi_{\ell}\right)$ is also localized in this region. With these definitions, the condition we must verify is

$[\mathbf{H 1}]\left(\gamma_{0}, \ell_{0}\right)$. There exist $\gamma_{0}>0$ and $\ell_{0}>>1$, such that $\gamma_{0} \ell_{0}>>1$, and

$$
\mathbb{P}\left\{\left\|W\left(\chi_{\ell}\right) R_{\Lambda_{\ell}}(E+i \varepsilon) \chi_{\ell / 3}\right\| \leq e^{-\gamma_{0} \ell_{0}} \|\right\} \geq 1-\ell_{0}^{-\xi},
$$

for $E$ near the band edges $\widetilde{B}_{ \pm}$and for some $\xi>2 d$.

We do this in two steps. We first prove that for $\delta_{\Lambda}>0$ small, $\operatorname{dist}\left(\sigma\left(H_{\Lambda}\right), \widetilde{B}_{ \pm}\right)>$ $\delta_{\Lambda}$, with good probability. We can then apply the Combes-Thomas result of Appendix 2 to conclude exponential decay at energies $E \in\left(\widetilde{B}_{-}-\delta_{\Lambda} / 2, \widetilde{B}\right) \cup\left(\widetilde{B}_{+}, \widetilde{B}_{+}+\delta_{\Lambda} / 2\right)$ with a good probability. We then verify $[\mathrm{H} 1]\left(\gamma_{0}, \ell_{0}\right)$ for an appropriate choice of $\gamma_{0}$ and $\ell_{0}$.

We now discuss the location of the spectrum of the finite-volume Hamiltonians $H_{\Lambda}$ in the unperturbed spectral gap. Recall that by (H8) the family $\left\{H_{\omega}\right\}$ has an almost sure spectrum $\Sigma$ with an open spectral gap $\left(\tilde{B}_{-}, \tilde{B}_{+}\right)$. The probability space for the models is $\Omega=(\operatorname{supp} g)^{\mathbb{Z}^{d}}$. 
Lemma 6.1. Suppose $\mu \equiv \mu_{\Lambda, \omega_{0}} \in \sigma_{d}\left(H_{\Lambda, \omega_{0}}\right) \cap\left(B_{-}, B_{+}\right)$, for some $\omega_{0} \in \Omega$, then $\mu \in \Sigma$.

Proof: Let $\psi_{\omega_{0}}$ be an eigenfunction of $H_{\Lambda, \omega_{0}}$ with eigenvalue $\mu_{\Lambda, \omega_{0}} \equiv \mu: H_{\Lambda, \omega_{0}} \psi_{\omega_{0}}=$ $\mu \psi_{\omega_{0}},\left\|\psi_{\omega_{0}}\right\|=1$. For any $R$ such that $\Lambda \subset \subset \Lambda_{R}$, and for any $\nu>0$, we define the following events:

$$
I_{R, \nu} \equiv\left\{\omega \in \Omega|| \omega_{0}-\omega_{i} \mid \leq \nu\left(6|\Lambda|\|u\|_{\infty}\right)^{-1}, \forall i \in \widetilde{\Lambda}\right\},
$$

and

$$
E_{R, \nu} \equiv\left\{\omega \in \Omega|| \omega_{i} \mid<\nu\left(6\left|\widetilde{\Lambda}_{R} \backslash \widetilde{\Lambda}\right|\|u\|_{\infty}\right)^{-1}, \forall i \in \widetilde{\Lambda}_{R} \backslash \widetilde{\Lambda}\right\}
$$

Set $B_{R, \nu} \equiv I_{R, \nu} \cap E_{R, \nu}$. Let $\chi \in C^{2}$ be a smoothed characteristic function with supp $\chi \subset \Lambda_{2}, \chi \leq 1$, and $\chi \mid \Lambda_{1}=1$. For $R>1$, set $\chi_{R}(x) \equiv \chi\left(R^{-1} x\right)$ so that $\left\|\partial^{\alpha} \chi_{R}\right\|=\mathcal{O}\left(R^{-|\alpha|}\right)$, for $|\alpha|=0,1,2$. Choose $R_{1}$ sufficiently large so $\left\|\chi_{R_{1}} \psi_{\omega_{0}}\right\|>$ $\frac{1}{2}$, and for $R>R_{1}$ define $\psi_{R} \equiv\left\|\chi_{R} \psi_{\omega_{0}}\right\|^{-1} \chi_{R} \psi_{\omega_{0}}$ so $\left\|\psi_{R}\right\|=1$. Then, by the definition of $\psi_{R}$ and the local Hamiltonians,

$$
\left(H_{\omega}-\mu\right) \psi_{R}=\left(H_{\Lambda, \omega_{0}}-\mu\right) \psi_{R}+\sum_{i \in \tilde{\Lambda}}\left(\omega_{i}-\omega_{0}\right) u_{i} \psi_{R}+\sum_{i \in \widetilde{\Lambda}_{R} \backslash \Lambda} \omega_{i} u_{i} \psi_{R},
$$

and it follows that for all $\omega \in B_{R, \nu}$

$$
\left\|\left(H_{\omega}-\mu\right) \psi_{R}\right\| \leq 2\left\|\left[H_{0}, \chi_{R}\right] \psi_{\omega_{0}}\right\|+\frac{1}{3} \nu
$$

The commutator is estimated as follows. As $H_{0}=(p-A)^{2}+V_{0}$, we have

$$
\left[(p-A)^{2}, \chi_{R}\right] \psi_{\omega_{0}}=-2 i \Delta \chi_{R}(p-A) \psi_{\omega_{0}}-\left(\Delta \chi_{R}\right) \psi_{\omega_{0}} .
$$

Now $\psi_{\omega_{0}}$ is an eigenfunction of $H_{\Lambda, \omega_{0}}$ and, in particular, $\psi_{\omega_{0}} \in D\left(H_{0}\right)$, so

$$
(p-A)_{j} \psi_{\omega_{0}}=(p-A)_{j}\left(H_{0}-z\right)^{-1}\left(\mu-z-V_{\Lambda, \omega_{0}}\right) \psi_{\omega_{0}} .
$$

Setting $z=i \delta, \delta>0$, we obtain

$$
\left\|(p-A)_{j} \psi_{\omega_{0}}\right\| \leq \delta^{-1}\left\|\mu-i \delta-V_{\Lambda, \omega_{0}}\right\|_{\infty} .
$$

which is independent of $R$. Hence, by taking $R$ sufficiently large, it follows from (6.5) that

$$
\left\|\left(H_{\omega}-\mu\right) \psi_{R}\right\| \leq \frac{2}{3} v .
$$

This shows that for any $\nu>0, \sigma\left(H_{\omega}\right) \cap[\mu-\nu, \mu+\nu] \neq \emptyset$ with probability $\mathbb{P}\left(B_{R, \nu}\right)=$ $\mathbb{P}\left(E_{R, \nu}\right) \mathbb{P}\left(I_{R, \nu}\right)>0$. Since the spectrum of $\left\{H_{\omega}\right\}$ is deterministic, this implies $\mu \in \Sigma$.

Lemma 6.2. Let $\mu_{\Lambda, \omega} \equiv \mu \in \sigma_{d}\left(H_{\Lambda, \omega}\right) \cap\left(B_{-}, B_{+}\right)$, with eigenfunction $\phi_{\omega},\left\|\phi_{\omega}\right\|=$ 1. Assume that $V_{\Lambda, \omega} \geq 0$. Then we have

$$
\left\langle\phi_{\omega}, V_{\Lambda, \omega} \phi_{\omega}\right\rangle \geq\left[\operatorname{dist}\left(\mu, \sigma\left(H_{0}\right)\right)\right]^{2} M_{\infty}^{-1} .
$$

Proof: Since $M_{\infty} V_{\Lambda, \omega} \geq\left(V_{\Lambda, \omega}\right)^{2}$ under the hypothesis that $V_{\Lambda, \omega} \geq 0$, we have

$$
\begin{aligned}
\left\langle\phi_{\omega}, V_{\Lambda, \omega} \phi_{\omega}\right\rangle & =M_{\infty}^{-1}\left\langle\phi_{\omega}, M_{\infty} V_{\Lambda, \omega} \phi_{\omega}\right\rangle \\
& \geq M_{\infty}^{-1}\left\|V_{\Lambda, \omega} \phi_{\omega}\right\|^{2} .
\end{aligned}
$$

The eigenvalue equation gives $V_{\Lambda, \omega} \phi_{\omega}=-\left(H_{0}-\mu\right) \phi_{\omega}$, so that

$$
\begin{aligned}
\left\langle\phi_{\omega}, V_{\Lambda, \omega} \phi_{\omega}\right\rangle & \geq M_{\infty}^{-1}\left\|\left(H_{0}-\mu\right) \phi_{\omega}\right\|^{2} \\
& \geq M_{\infty}^{-1}\left[\operatorname{dist}\left(\sigma\left(H_{0}\right), \mu\right)\right]^{2},
\end{aligned}
$$


which proves the estimate.

Proposition 6.1. Let $\delta_{ \pm} \equiv \frac{1}{2}\left|\widetilde{B}_{ \pm}-B_{ \pm}\right|$, and for any $0<\delta<\frac{1}{2} M_{\infty}^{-1} \min \left(\delta_{+}, \delta_{-}\right)$, assume that $\omega_{i}<\left(1-\delta M_{\infty}\left[\min \left(\delta_{+}, \delta_{-}\right)\right]^{-2}\right) M, \forall i \in \widetilde{\Lambda}$. Then we have

$$
\sup \left\{\sigma\left(H_{\Lambda, \omega}\right) \cap\left(-\infty, \widetilde{B}_{-}\right)\right\}<\widetilde{B}_{-}-\delta
$$

and

$$
\inf \left\{\sigma\left(H_{\Lambda, \omega}\right) \cap\left(\widetilde{B}_{+}, \infty\right)\right\}>\widetilde{B}_{+}+\delta .
$$

Proof: Without loss of generality, we assume $H_{\Lambda, \omega}$ has an eigenvalue $\mu_{\Lambda, \omega} \equiv$ $\mu \in\left[\widetilde{B}_{-}-\delta, \widetilde{B}_{-}\right]$. Furthermore, we can assume that $V_{\Lambda, \omega} \geq 0$, since by Lemma 6.1 , we always have $\mu \leq \widetilde{B}_{-}$and the eigenvalues of $H_{\Lambda, \omega}$ are increasing functions of the coupling constants $\left\{\omega_{i} \mid i \in \widetilde{\Lambda}\right\}$. This fact follows, for example, from the Feynman-Hellman formula, Theorem 4.1, and the positivity of $u$. Indeed, if $\phi_{\omega}$ is an eigenfunction of $H_{\Lambda, \omega}$, so that $H_{\Lambda, \omega} \phi_{\omega}=\mu \phi_{\omega}$, then

$$
\begin{aligned}
\frac{\partial \mu_{\Lambda, \omega}}{\partial \omega_{i}} & =\left\langle\phi_{\omega}, \frac{\partial H_{\Lambda, \omega}}{\partial \omega_{i}} \phi_{\omega}\right\rangle \\
& =\left\langle\phi_{\omega}, u_{i} \phi_{\omega}\right\rangle>0 .
\end{aligned}
$$

The family $T(\theta) \equiv H_{0}+\theta V_{\Lambda, \omega}$, for $\theta$ in a small neighborhood of $\theta_{0}=1$, is an analytic type $A$ family which is self-adjoint for $\theta$ real. If $\mu$ has multiplicity $m$, there are at most $m$ functions $\mu^{(k)}(\theta)$, analytic in $\theta$ for $\theta$ near $\theta_{0}=1$, and which satisfy $\lim _{\theta \rightarrow \theta_{0}=1} \mu^{(k)}(\theta)=\mu$. Let $\phi^{(k)}(\theta)$ be an eigenfunction for $\mu^{(k)}(\theta)$, with $\left\|\phi^{(k)}(\theta)\right\|=1$ for $\theta$ real and $|\theta-1|$ small. Applying the Feynman-Hellman formula again, we find

$$
\begin{aligned}
\frac{d \mu^{(k)}(\theta)}{d \theta} & =\left\langle\phi(\theta), V_{\Lambda, \omega} \phi(\theta)\right\rangle \\
& =\theta^{-1}\left\langle\phi(\theta),\left(\theta V_{\Lambda, \omega}\right) \phi(\theta)\right\rangle .
\end{aligned}
$$

We now assume $\omega_{i}<\left(1-\delta M_{\infty}\left[\min \left(\delta_{+}, \delta_{-}\right)\right]^{-2}\right) M, \forall i \in \widetilde{\Lambda}$, and fix

$$
\begin{aligned}
\theta_{1}=\min _{i \in \tilde{\Lambda}}\left(\frac{M}{\omega_{i}}\right) & \geq\left(1-\delta M_{\infty}\left[\min \left(\delta_{+}, \delta_{-}\right)\right]^{-2}\right)^{-1} \\
& >1 .
\end{aligned}
$$

Applying Lemma 6.2 to $V_{\Lambda, \omega}$ under these conditions yields

$$
\frac{d \mu^{(k)}(\theta)}{d \theta} \geq \theta^{-1} M_{\infty}^{-1}\left[\operatorname{dist}\left(\mu^{(k)}(\theta), \sigma\left(H_{0}\right)\right)\right]^{2},
$$

Upon integrating over $\left[1, \theta_{1}\right]$, we get, by monotonicity of $\mu^{(k)}(\theta)$ :

$$
\begin{aligned}
\mu^{(k)}\left(\theta_{1}\right) & \geq \mu+\left(\log \theta_{1}\right) M_{\infty}^{-1} \min \left\{\left[\operatorname{dist}\left(\mu^{(k)}\left(\theta_{1}\right), \sigma\left(H_{0}\right)\right)\right]^{2},\left[\operatorname{dist}\left(\mu, \sigma\left(H_{0}\right)\right)\right]^{2}\right\} \\
& \geq \mu+\delta>\widetilde{B}_{-} .
\end{aligned}
$$

This shows that $\left(H_{0}+\sum_{i \in \widetilde{\Lambda}} M u_{i}\right)$ has an eigenvalue outside of $\Sigma$ which contradicts Lemma 6.1.

This proposition is the main technical result. We can now easily compute the probability that $\operatorname{dist}\left(\sigma\left(H_{\Lambda, \omega}\right), \widetilde{B}_{ \pm}\right)>\delta_{\Lambda}$. 
Corollary 6.1. For $0<\delta<\frac{1}{2} M_{\infty}^{-1} \min \left(\delta_{+}, \delta_{-}\right)$, we have

$$
\sup \left\{\sigma\left(H_{\Lambda, \omega}\right) \cap\left(-\infty, \widetilde{B}_{-}\right)\right\}<\widetilde{B}_{-}-\delta
$$

and

$$
\inf \left\{\sigma\left(H_{\Lambda, \omega}\right) \cap\left(\widetilde{B}_{+}, \infty\right)\right\}>\widetilde{B}_{+}+\delta
$$

with a probability larger than

$$
1-|\Lambda| \max _{X=m, M}\left|\int_{1-\delta M_{\infty}\left[\min \left(\delta_{+}, \delta_{-}\right)\right]^{-2} X}^{X} g(s) d s\right|
$$

Proof: The probability that $\omega_{i}<\left(1-\delta M_{\infty}\left[\min \left(\delta_{+}, \delta_{-}\right)\right]^{-2}\right) M, \forall i \in \widetilde{\Lambda}$, is given by $\left[1-\int_{\left(1-\delta M_{\infty} \Delta^{-2}\right) M}^{M} h(s) d s\right]^{|\Lambda|}$. The corollary now follows by expanding this probability and from Proposition 6.1.

6.3. Verification of $[\mathbf{H 1}]\left(\gamma_{0}, \ell_{0}\right)$. We verify $[\mathrm{H} 1]\left(\gamma_{0}, \ell_{0}\right)$ by combining Corollary 6.1 on the location of the spectrum of $H_{\Lambda_{\ell}, \omega}$ and the exponential decay estimate of [8]. We note that hypothesis (H6) on the decay of the tail of the density $h$ near the endpoints of its support $m$ and $M$ is essential in order to control the probability in Corollary 6.1. We first give the decay estimate for the localized resolvent and then comment on the gradient term.

Proposition 6.2. Let $\chi_{i}, i=1,2$, be two functions with $\left\|\chi_{i}\right\|_{\infty} \leq 1, \operatorname{supp} \chi_{1} \subset$ $\Lambda_{\ell / 3}$ and supp $\chi_{2}$ localized near $\partial \Lambda_{\ell}$ and $\delta_{ \pm} \equiv \frac{1}{2}\left|\widetilde{B}_{+}-\widetilde{B}_{-}\right|$. For $\beta>0$ as in (H6), consider any $\nu>0$ such that $0<\nu<4 \beta(2 \beta+3 d)^{-1}$. Then $\exists \ell_{0}^{\star} \equiv$ $\ell_{0}^{\star}\left(M_{\infty}, \delta_{+}, \delta_{-}, M\right)$ such that $\forall \ell_{0}>\ell_{0}^{\star}$ and $\forall E \in\left(\widetilde{B}_{-}-\ell_{0}^{\nu-2}, \widetilde{B}_{-}\right] \cup\left[\widetilde{B}_{+}, \widetilde{B}_{+}+\ell_{0}^{\nu-2}\right)$,

$$
\sup _{\varepsilon>0}\left\|\chi_{2} R_{\Lambda_{\ell_{0}}}(E+i \varepsilon) \chi_{1}\right\| \leq e^{-\ell_{0}^{\nu / 3}}
$$

with probability $\geq 1-\ell_{0}^{-\xi}$, for some $\xi>2 d$.

Proof: From Corollary 6.1 and (H7), we compute the probability that $\sigma\left(H_{\left.\Lambda_{\ell_{0}, \omega}\right)}\right.$ is at a distance $\delta=2 \ell_{0}^{\nu-2}$ from $\widetilde{B}_{ \pm}$,

$\mathbb{P}\left\{\operatorname{dist}\left(\sigma\left(H_{\Lambda_{\ell_{0}}, \omega}\right), \widetilde{B}_{ \pm}\right)>2 \delta\right\} \geq 1-\ell_{0}^{d}\left(2 \ell_{0}^{\nu-2} M_{\infty}\left[\min \left(\delta_{+}, \delta_{-}\right)\right]^{-2} X\right)^{3 d / 2+\beta}$

where $X=m$ for $\widetilde{B}_{-}$and $X=M$ for $\widetilde{B}_{+}$. A simple computation shows that the right side of (6.9) is bounded below by $1-\ell_{0}^{-\xi}$ for some $\xi>2 d$ provided $\nu$ satisfies $0<\nu<4 \beta(2 \beta+3 d)^{-1}$. We now apply the Combes-Thomas exponential decay estimate [8] $H_{\Lambda_{\ell_{0}}, \omega}$. Since $\operatorname{dist}\left(\operatorname{supp} \chi_{2}\right.$, supp $\left.\chi_{1}\right) \geq \ell_{0} / 3$ (in dimension $d>9$, this is no longer true; one has to replace $\ell_{0} / 3$ by $\ell_{0} /(3 \sqrt{d})$, for the diameter of the inner cube), we obtain

$$
\begin{aligned}
\left\|\chi_{2} R_{\Lambda_{\ell_{0}}}(E+i \varepsilon) \chi_{1}\right\| & \leq C_{2} \sup \left(\left|\widetilde{B}_{+}-\widetilde{B}_{-}\right|^{-1}, \ell_{0}^{2-\nu}\right) \\
& \times e^{-i n f\left(\alpha_{0}, C_{1} \ell_{0}^{\nu / 2-1}\left|\widetilde{B}_{+}-\widetilde{B}_{-}\right|^{1 / 2}\right) \ell_{0} / 6}
\end{aligned}
$$

The result follows by taking $\ell_{0}$ large.

Corollary 6.2. There exists a length scale $\ell_{0}^{\star}>0$ such that $\forall \ell_{0}>\ell_{0}^{\star}$, hypothesis [H1] $\left(\gamma_{0}, \ell_{0}\right)$ holds $\forall E \in\left(\widetilde{B}_{-}-\ell_{0}^{\nu-2}, \widetilde{B}_{-}\right] \cup\left[\widetilde{B}_{+}, \widetilde{B}_{+}+\ell_{0}^{\nu-2}\right)$ and any $\nu$ satisfying $0<\nu<4 \beta(2 \beta+3 d)^{-1}, \beta$ as in $(\mathrm{H} 6)$. 
Proof: As in (8.23) of chapter 8, we write

$$
\begin{aligned}
\left\|W\left(\chi_{\ell, v}\right) R_{\Lambda_{\ell}} \chi_{\ell / 3}\right\| \leq & \left\|\left(\Delta \chi_{\ell, v}\right) R_{\Lambda_{\ell}} \chi_{\ell / 3}\right\| \\
& +2 \sum_{j=1}^{d}\left\|\left(\partial_{j} \chi_{\ell, v}\right)(p-A)_{j} R_{\Lambda_{\ell}} \chi_{\ell / 3}\right\|
\end{aligned}
$$

for a function $\chi_{\ell, v}$ localized within distance $v$ of $\partial \Lambda_{\ell}$. Let $\chi_{i}, i=1,2$, be smooth functions such that $\chi_{i} \chi_{\ell, v}=\chi_{\ell, v}, \chi_{2} \chi_{1}=\chi_{1}$, and $\operatorname{supp} \chi_{i}$ is localized within a distance $2 v$ for $i=1$ and $3 v$ for $i=2$, of $\partial \Lambda_{\ell}$. Then, we write for each $j$ and any $u \in L^{2}\left(\mathbb{R}^{d}\right)$,

$$
\begin{aligned}
\left\|\left(\partial_{j} \chi_{\ell, v}\right)(p-A)_{j} R_{\Lambda_{\ell}} u\right\|^{2} & \leq C_{0}\left\langle(p-A)_{j} R_{\Lambda_{\ell}} u, \chi_{1}(p-A)_{j} \chi_{2} R_{\Lambda_{\ell}} u\right\rangle \\
& \leq C_{0}\left\|\chi_{2} R_{\Lambda_{\ell}} u\right\|\left\|(p-A)_{j} \chi_{1}(p-A)_{j} R_{\Lambda_{\ell}} u\right\|
\end{aligned}
$$

Taking $u=\chi_{\ell / 3} f$, we see that (6.10) is bounded above as in Proposition 6.2 (taking $\ell_{0}^{\star}$ larger) provided we have $\left\|(p-A)^{2} R_{\Lambda_{\ell_{0}}} u\right\|$ bounded. This follows with a probability $\geq 1-\ell_{0}^{-\xi}$, since $V_{0}$ is relatively bounded and $V_{\omega}^{\Lambda_{\ell_{0}}}$ is bounded.

\section{Random Magnetic Schrödinger Operators and the Integer Quantum Hall Effect}

7.1. Overview: Integer Quantum Hall Effect. This chapter is devoted to the study of the family of random Schrödinger operators describing an electron moving in two-dimensions and subject to a random potential and a constant, transverse magnetic field, and the application of this model to the integral quantum Hall Effect (IQHE). The classic Hall effect for a thin conductor of width $\delta$ and infinite spatial extend in the $\left(x_{1}, x_{2}\right)$-directions, may be described as follows (we follow [15]). The conductor is viewed as a collection of fixed, positively charges centers and a sea of negatively charged, noninteracting, electrons with charge $q$ and density $n$. The sample is subject to a transverse, constant magnetic field $B=(0,0, B)$. In this ideal experiment, suppose that a constant electric field $E=(E, 0,0)$ is applied in the $x_{1}$-direction. This creates a current $J$ of electrons moving in the $x_{1}$-direction. The Lorentz force, proportional to $J \times B$, acts on the electrons in the $x_{2}$-direction. As a result, a potential difference develops in the $x_{2}$-direction with a corresponding electric field, the Hall field $E_{H}$, in the $x_{2}$-direction. If the system is in equilibrium, the force on the electrons from $E_{H}$ and the Lorentz force must balance, giving rise to the equation

$$
n q E_{H}+J \times B=0 .
$$

We can solve this equation by taking the curl with the magnetic field $B$ and obtain,

$$
J=\sigma E_{H},
$$

Restricting to the $\left(x_{1}, x_{2}\right)$-plane, and changing to surface density $\delta n$, the $2 \times 2$ conductivity tensor $\sigma$ has zero diagonal elements and nonvanishing off-diagonal elements given by

$$
\sigma_{21}=\frac{n q \delta}{B}=-\sigma_{12}
$$

We call $\sigma_{12}$ the Hall conductance $\sigma_{H}$. It is proportional to the electron density in the plane $n \delta$. This is the classic Hall effect and has been observed since the end of 
the nineteenth century. For comparison with the IQHE, we define the filling factor by

$$
\nu \equiv \frac{n \delta h}{B q},
$$

where $h$ is Planck's constant. In terms of this, the Hall conductance (7.3) is

$$
\sigma_{H}=\nu \frac{q^{2}}{h},
$$

which is proportional to the filling factor.

In the 1980's, experimental techniques had advanced to the point where the Hall effect experiments could be performed at very low temperatures. As is now widely known, the law in (7.3) is not observed, but rather von Klitzing and coworkers observed a quantization of the Hall conductivity. In particular, they found that

$$
\sigma_{H} \in \frac{q^{2}}{h} I N,
$$

That is, the Hall conductance is quantized in integer multiples of $q^{2} / h$. As the filling factor increases, the Hall conductance remains constant and then increases by integer amounts. The experiments are extremely accurate. This behavior of the Hall conductance can now be explained in terms of the Kubo formula for the conductance and properties of one-particle random Schrödinger operators, see, for example [15].

7.2. Landau Hamiltonians with Random Potentials. We consider a oneparticle Hamiltonian which describes an electron in two-dimensions $\left(x_{1}, x_{2}\right)$ subject to a constant magnetic field of strength $B>0$ in the perpendicular $x_{3}$-direction, and a random potential $V_{\omega}$. The Hamiltonian $H_{\omega}$ has the form

$$
H_{\omega}=(p-A)^{2}+V_{\omega}=H_{A}+V_{\omega}
$$

on the Hilbert space $L^{2}\left(\mathbb{R}^{2}\right)$, where $p \equiv-i \nabla$, and the vector potential $A$ is

$$
A=\frac{B}{2}\left(x_{2},-x_{1}\right),
$$

so the magnetic field $B=\nabla \times A$ is in the $x_{3}$-direction. The random potential $V_{\omega}$ is Anderson-like having the form

$$
V_{\omega}(x)=\sum_{i \in \mathbb{Z}^{2}} \omega_{i} u(x-i) .
$$

We denote by $H_{A} \equiv(p-A)^{2}$, the Landau Hamiltonian. As is well-known, the spectrum of $H_{A}$ consists of an increasing sequence $\left\{E_{n}(B)\right\}$ of eigenvalues, each of infinite multiplicity, given by

$$
E_{n}(B)=(2 n+1) B, n=0,1,2, \ldots
$$

We will call $E_{n}(B)$ the $\mathrm{n}^{\text {th }}$ Landau level and denote by $P_{n}$ the projection onto the corresponding subspace. The orthogonal projection is denoted by $Q_{n} \equiv 1-P_{n}$.

This family of random Schrödinger operators plays a key role in the theory of the integer quantum Hall effect (IQHE) described in section 7.1. Several recent discussions of the integer quantum Hall effect (IQHE) $[\mathbf{1 4}, \mathbf{1 5}, \mathbf{7 0}, \mathbf{9 6}, \mathbf{1 0 8}, \mathbf{1 0 9}, \mathbf{1 2 6}]$ require certain spectral properties of this family of random, one-particle Schrödinger operators. As proved in [15], the one-particle model of a free electron moving in two 
dimensions in the presence of a constant, transverse magnetic field will not exhibit the IQHE. Rather, the conductance follows the classical law (7.3). At very low temperatures, when dissipative effects are small, it is commonly believed that random impurities in the sample provide a necessary mechanism for the quantization of the Hall conductance and the appearance of the plateaus. The key hypothesis on the spectral properties of one-particle Hamiltonian is the existence of regions of localized states between the Landau levels.

In this chapter, we discuss the existence of localized states for Landau Hamiltonians with random perturbations. We provide the necessary arguments for the Wegner estimate and the initial length scale estimate that are necessary for the proof of the existence of localized states at energies between the Landau levels $E_{n}(B)$, and to within $\mathcal{O}\left(\log B B^{-1}\right)$ of the Landau energies, for fixed disorder and large magnetic field depending on the Landau level $n$. The actual proof that these estimates suffice to prove Anderson localization requires a more refined MSA than that presented in chapter 8 here and we refer the reader to the original references $[62,63]$.

There are two cases to consider. In the first case, the random potential is bounded. For bounded perturbations, a large magnetic field means that there are spectral gaps between the Landau bands of length $\mathcal{O}(B)$ since the Landau levels broaden no more than $\left\|V_{\omega}\right\|_{\infty}$, which is bounded independent of $B$. The corresponding eigenfunctions for energies in the localized regime decay exponentially with respect to distance and the magnetic field strength $B$. Kunz's proof $[\mathbf{9 6}]$ of the IQHE requires the existence of spectral gaps and localized states near the bandedges with finite localization length. A consequence of his analysis is a proof that the localization length diverges at some energy in each band. It is believed that for neutral samples, the localization length should diverge precisely at the Landau energy.

In the second case, the random potential is unbounded. Typically, the spectral gaps no longer exist, having been completely filled-in by spectrum. The importance of this case is discussed in a review article by Bellissard, van Elst, and SchulzBaldes [15]. These authors point out that in experiments, the disorder is strong enough to fill the gap between the Landau levels. In their proof of the IQHE, they require regions of localized states between the Landau energies in order that the quantum Hall conductivity exhibits a plateau region. They do not require a spectral gap. They prove that if the quantum Hall conductivity jumps by an integer (as a function of the filling factor), then the localization length must diverge at some energy between the localized state regions.

The second result presented in this chapter concerns the integrated density of states. Using a proof simpler than the one presented in chapter 4, we prove that, in general, the integrated density of states (IDS) is Lipschitz continuous at all energies but the Landau energies. If, in addition, the support of the single site potential $u$ includes the unit cell, then the IDS is Lipschitz continuous at all energies. It is now known that the IDS for the random Landau Hamiltonian is Lipschitz continuous at all energies, $[\mathbf{2 8}, \mathbf{2 9}, \mathbf{6 6}]$, but the proof requires a different version of the quantitative unique continuation principle. Much less is known about the regularity of the IDS. W. M. Wang $[\mathbf{1 3 4}, \mathbf{1 3 5}]$ proved the smoothness of the IDS at energies away from the Landau energies provided the single-site probability density is smooth. 
From the point of view of models, the randomly perturbed Landau Hamiltonian is interesting because the proof of localization requires the use of nontrivial results from the theory of bond percolation. In particular, the critical probability for bond percolation, the probability above which an infinite cluster exists almost surely, enters in a key way.

7.3. A Description of the Model and the Main Results. We make the following assumptions on the single-site potential $u$ and the coupling constants $\left\{\omega_{i}\right\}$ appearing in the Anderson-type potential (7.7).

(V1) $u \geq 0, u \in C^{2}$, supp $u \subset B(0,1 / \sqrt{2})$, and $\exists C_{0}>0$ and $r_{0}>0$ s.t. $u \mid B\left(0, r_{0}\right)>C_{0}$.

(V2) $\left\{\omega_{i}\right\}$ is an independent, identically distributed family of random variables with common distribution $g \in L^{\infty}([-M, M])$, for some $0<M \leq \infty$, s.t. $g(-\omega)=g(\omega), g(\omega)>0$ Lebesgue a.e. $\omega \in[-M, M]$, and for some $\epsilon>0$, $\sup _{\omega \in \mathbb{R}}\left\{\omega^{3+\epsilon} g(\omega)\right\}<\infty$.

The condition on the decay of $g$ implies that the first two moments are finite, i.e. $\int|\omega|^{k} g(\omega) d \omega<\infty$, for $k=0,1,2$. The above condition does not require any differentiability of the density $g$. If we require $g$ to be twice differentiable, we can replace (V2) by another condition.

(V2)' $\left\{\omega_{i}\right\}$ is an independent, identically distributed family of random variables with common distribution $g \in C^{2}([-M, M])$, for some $0<M \leq \infty$, s.t. $g(-\omega)=g(\omega), g(\omega)>0$ Lebesgue a.e. $\omega \in[-M, M],\left\|g^{(p)}\right\|_{1}<\infty$, for $p=0,1,2$, and $\int_{-M}^{M} \omega^{2} g(\omega)<\infty$.

We remark for future use that either condition (V2) or (V2)' implies that

$$
\mathbb{P}\{\omega \geq \xi>0\}=\mathcal{O}\left(\xi^{-N}\right) \text {, for all } \xi>0 \text { large and some } N \geq 2 .
$$

An important example is the case of Gaussian distributed coupling-constants for which $g(\omega)=(\alpha \pi)^{-1 / 2} e^{-\alpha \omega^{2}}$, for some $\alpha>0$.

Our main theorem concerning localization for random Landau Hamiltonians is the following $[\mathbf{2 6}, \mathbf{6 3}, \mathbf{1 3 3}]$.

THEOREM 7.1. Let $H_{\omega}$ be the family given in (7.7) with vector potential $A$ as in (7.8) with $B>0$, and the random potential $V_{\omega}$ as in (7.9), and satisfying (V1) and either (V2) or (V2)' Let $I_{n}(B)$ denote the unbounded set of energies

$I_{n}(B) \equiv\left(-\infty, B-\mathcal{O}\left(\log B \cdot B^{-1}\right)\right] \cup \bigcup_{j=0}^{n}\left[E_{j}(B)+\mathcal{O}\left(\log B \cdot B^{-1}\right), E_{j+1}(B)-\mathcal{O}\left(\log B \cdot B^{-1}\right)\right]$,

where the term $\mathcal{O}\left(\log B \cdot B^{-1}\right)$ depends on $n$. For each integer $n>0$, there exists $B_{n} \gg 0$ such that for $B>B_{n}$,

$$
\Sigma \cap I_{n}(B)
$$

is pure point and the corresponding eigenfunctions decay exponentially.

We remark that we can also prove localization at energies between the Landau levels for fixed, nonzero $B>0$ for the family $H_{\omega}(g) \equiv H_{A}+g V_{\omega}$, with a coupling constant $g>0$, provided that we work in the small coupling regime. This result does not require percolation theory, but we cannot control the spectrum to within $\mathcal{O}\left(B^{-1}\right)$ of the Landau levels. We refer the reader to section 6 of $[\mathbf{8}]$ where results of this type are proved in general situations. As with previous work, the Wegner estimate for the finite-area Hamiltonians allows us to control the integrated density 
of states. However, we no longer need to take $B$ large as in $[\mathbf{2 6}]$. The proof of the following theorem is given in $[\mathbf{2 8}]$ based on results in $[\mathbf{2 9 , 6 6 ]}$.

TheOREm 7.2. Let $H_{\omega}$ be the family given in (7.7) with vector potential $A$ as in (7.8) with $B>0$, and the random potential $V_{\omega}$ as in (7.9) and satisfying (V1) and either (V2) or (V2)'. Then, the integrated density of states is locally uniformly Lipschitz continuous on $\mathbb{R}$.

7.4. The Wegner Estimate. The Wegner estimate for this model follows the same lines of the proof of the Wegner estimate given in chapter 4. There are some simplifications which we will mention due to the explicit structure of the unperturbed Landau Hamiltonian $H_{A}$. As these are of interest in themselves, we give a proof of the Wegner that works for all energies away from the Landau levels. The proof of the Lipschitz continuity of the IDS at all energies is given in [28]. We will use the spectral averaging result of chapter 3 . The local Hamiltonians $H_{\Lambda}$ are defined as in chapter 4 . For a bounded region $\Lambda \subset \mathbb{R}^{2}$, we define the local potential $V_{\Lambda}$ by $V_{\omega} \mid \Lambda$. The local Hamiltonian is $H_{\Lambda} \equiv H_{A}+V_{\Lambda}$. As discussed in chapter 4, the essential spectrum of $H_{A}$ is invariant under this perturbation. The Wegner estimate for these local Hamiltonians takes the following form.

THEOREM 7.3. There exists a constant $B_{0}>0$ and a constant $C_{W}>0$ such that for all $B>B_{0}$ and for any $E \notin \sigma\left(H_{A}\right)$ and $\eta>0$ so that $[E-\eta, E+\eta] \cap \sigma\left(H_{A}\right)=\emptyset$, we have,

$$
\mathbb{P}_{\Lambda}\left\{\operatorname{dist}\left(\sigma\left(H_{\Lambda}\right), E\right)<\eta\right\} \leq C_{W}\left[\operatorname{dist}\left(\sigma\left(H_{A}\right), E\right)-\delta\right]^{-2}\|g\|_{\infty} \eta B|\Lambda| .
$$

In the proof of this theorem, the exponential decay of the resolvent of the unperturbed Hamiltonian is replaced by the explicit exponential decay of the projectors onto the the Landau levels when localized between disjoint sites. Let $P_{n}$ be the orthogonal projection on the $\mathrm{n}^{\text {th }}$ Landau level of $H_{A}$. The projector $P_{n}$ has an integral kernel given by

$$
P_{n}(x, y)=B e^{-i \frac{B}{2} x \wedge y} p_{n}\left(B^{1 / 2}(x-y)\right),
$$

where $p_{n}(x)$ is of the form

$$
p_{n}(x)=\left\{\mathrm{n}^{\text {th }} \text { degree polynomial in } x\right\} e^{-|x|^{2} / 2},
$$

and independent of $B$. We define the projector $Q_{n}$ as $Q_{n} \equiv 1-P_{n}$. We will make repeated use of the following two elementary lemmas. The proof of Lemma 7.4 follows by direct calculation using the kernel (7.12)-(7.13).

LEMMA 7.4. Let $\chi_{1}, \chi_{2}$ be functions of disjoint, not necessarily compact, support with $\left|\chi_{i}\right| \leq 1$, and let $\delta \equiv \operatorname{dist}\left(\operatorname{supp} \chi_{1}\right.$, supp $\left.\chi_{2}\right)>0$. Then,

(1) ||$\chi_{1} P_{n} \chi_{1} \|_{1} \leq C_{n} B\left|\operatorname{supp} \chi_{1}\right|$;

(2) $\left\|\chi_{1} P_{n} \chi_{2}\right\|_{H S} \leq C_{n} B^{1 / 2} e^{-B \delta^{2} / 8} \inf \left\{\left|\operatorname{supp} \chi_{1}\right|,\left|\operatorname{supp} \chi_{2}\right|\right\}^{1 / 2}$,

where $C_{n}$ varies from line to line and depends only on $n$, and $H S$ denotes the Hilbert Schmidt norm.

The next lemma allows us to estimate the size of the overlap between the perturbed band given by the range of $E_{\Delta}$ and the orthogonal complement of the first unperturbed Landau level. 
LEMma 7.5. The restriction of the projection $Q_{0}$ to the first band satisfies

$$
\left\|E_{\Delta} Q_{0} E_{\Delta}\right\| \leq d_{\Delta}^{-2}\left(1-\left(2 d_{\Delta}\right)^{-1}|\Delta|\right)^{-2} M_{0}^{2},
$$

where $d_{\Delta} \equiv \operatorname{dist}\left(\sigma\left(H_{A}\right) \backslash\{B\}, \Delta\right)=\mathcal{O}(B)$, and $M_{0} \equiv\left\|V_{\omega}\right\|_{\infty} \leq M\|u\|_{\infty}$.

Proof. Let $E_{m} \in \Delta$ be the center of the interval. We then can write

$$
\begin{aligned}
E_{\Delta} Q_{0} E_{\Delta} & \leq\left[\operatorname{dist}\left(\sigma\left(H_{A}\right) \backslash\{B\}, \Delta\right)\right]^{-1}\left(E_{\Delta}\left(H_{A}-E_{m}\right) Q_{0} E_{\Delta}\right) \\
& \leq d_{\Delta}^{-1}\left\{E_{\Delta}\left(H_{\Lambda}-E_{m}\right) Q_{0} E_{\Delta}+E_{\Delta} V_{\Lambda} Q_{0} E_{\Delta}\right\} .
\end{aligned}
$$

This implies that

$$
\left\|E_{\Delta} Q_{0} E_{\Delta}\right\| \leq d_{\Delta}^{-1}\left\{|\Delta| / 2\left\|E_{\Delta} Q_{0} E_{\Delta}\right\|+M_{0}\left\|Q_{0} E_{\Delta}\right\|\right\} .
$$

Since $d_{\Delta}=\mathcal{O}(B)$, it is clear that for all $B$ sufficiently large $\left(2 d_{\Delta}\right)^{-1}|\Delta| \ll 1$, so

$$
\left\|E_{\Delta} Q_{0} E_{\Delta}\right\| \leq d_{\Delta}^{-1}\left(1-\left(2 d_{\Delta}\right)^{-1}|\Delta|\right)^{-1} M_{0}\left\|E_{\Delta} Q_{0} E_{\Delta}\right\|^{1 / 2},
$$

and the result follows.

We will compute the Wegner estimate under the assumption that the closest point in $\sigma\left(H_{A}\right)$ to the energy $E$ is the first Landau level $E_{0}(B)$. The arguments apply to any Landau level but the constants depend on the index $n$.

Proof of Theorem 7.3.

1. As in the proof of Theorem 4.3, we must estimate $\mathbb{E}_{\Lambda}\left(\operatorname{Tr} E_{\Delta}\right)$. Working with the first Landau level for simplicity, we separate the trace into a piece depending upon $P_{0}$, and one depending on $Q_{0}$,

$$
\operatorname{Tr} E_{\Delta}=\operatorname{Tr} E_{\Delta} P_{0} E_{\Delta}+\operatorname{Tr} E_{\Delta} Q_{0} E_{\Delta} .
$$

We make the simple estimate

$$
\operatorname{Tr} E_{\Delta} Q_{0} E_{\Delta} \leq\left\|E_{\Delta} Q_{0} E_{\Delta}\right\|\left(\operatorname{Tr} E_{\Delta}\right)
$$

where we used the fact that $E_{\Delta} Q_{0} E_{\Delta} \geq 0$. Now by Lemma 7.3, $\left\|E_{\Delta} Q_{0} E_{\Delta}\right\|=$ $\mathcal{O}\left(B^{-2}\right)$, so it follows that for all $B$ sufficiently large,

$$
\operatorname{Tr} E_{\Delta} \leq 2 \operatorname{Tr}\left(P_{0} E_{\Delta} P_{0}\right) .
$$

2. Let us now suppose inf $\Delta>B$ for definiteness. From (7.7), and positivity we obtain

$$
\begin{aligned}
\operatorname{Tr} E_{\Delta} P_{0} E_{\Delta} & \leq \operatorname{Tr}\left(E_{\Delta}\left(H_{\Lambda}-B\right) P_{0}\left(H_{\Lambda}-B\right) E_{\Delta}\right) \cdot[\operatorname{dist}(\Delta, B)]^{-2} \\
& \leq \operatorname{Tr}\left(P_{0} V_{\Lambda} E_{\Delta} V_{\Lambda} P_{0}\right) \cdot[\operatorname{dist}(\Delta, B)]^{-2} .
\end{aligned}
$$

We now expand the potential $V_{\Lambda}$, writing $V_{\Lambda}=\sum_{i} \omega_{i} u_{i}$ for short. The trace in (7.18) is

$$
\sum_{i, j} \omega_{i} \omega_{j} \operatorname{Tr}\left(P_{0} u_{i} E_{\Delta} u_{j} P_{0}\right),
$$

where $i, j \in \Lambda \cap \mathbb{Z}^{2}$. Defining $A^{i j} \equiv u_{i}^{1 / 2} A u_{j}^{1 / 2}$, for any $A \in B(\mathcal{H})$, we have from (7.7.19),

$$
\sum_{i, j} \omega_{i} \omega_{j} \operatorname{Tr}\left(P_{0}^{j i} E_{\Delta}^{i j}\right)
$$


We must estimate

$$
\begin{aligned}
\mathbb{E}_{\Lambda}\left(\sum_{i, j} \omega_{i} \omega_{j} \operatorname{Tr}\left(P_{0}^{j i} E_{\Delta}^{i j}\right)\right) & \leq \sum_{i, j} \mathbb{E}_{\Lambda}\left(\left|\omega_{i} \omega_{j}\right|\left|\operatorname{Tr}\left(P_{0}^{j i} E_{\Delta}^{i j}\right)\right|\right) \\
& \leq M^{2} \sum_{i, j} \mathbb{E}_{\Lambda}\left(\left|\operatorname{Tr}\left(P_{0}^{j i} E_{\Delta}^{i j}\right)\right|\right) .
\end{aligned}
$$

3. To estimate $\mathbb{E}_{\Lambda}\left(\left|\operatorname{Tr}\left(P_{0}^{j i} E_{\Delta}^{i j}\right)\right|\right)$, we write the spectral decomposition of the trace-class operator $P_{0}^{j i}$ as

$$
P_{0}^{j i}=\sum_{n=1}^{\infty} \mu_{n}\left\langle\psi_{n}, \cdot\right\rangle \phi_{n}
$$

where $\left\{\mu_{n}\right\}$ are the deterministic, non-negative eigenvalues of $\left|P_{0}^{j i}\right|$, so that $\sum_{n} \mu_{n}=$ $\left\|P_{0}^{j i}\right\|_{1}$, and the set $\left\{\psi_{n}\right\}$ (respectively, $\left\{\phi_{n}\right\}$ ) is the orthonormal basis of eigenvectors for $\left|P_{0}^{j i}\right|$ (respectively, $\left|\left(P_{0}^{j i}\right)^{*}\right|=\left|P_{0}^{i j}\right|$ ). Expanding the trace in (7.21) in this basis, we obtain,

$$
\begin{aligned}
\left|\operatorname{Tr}\left(P_{0}^{j i} E_{\Delta}^{i j}\right)\right| & \leq \sum_{n} \mu_{n}\left|\left\langle\psi_{n}, E_{\Delta}^{i j} \phi_{n}\right\rangle\right| \\
& \leq \frac{1}{2} \sum_{n} \mu_{n}\left(\left\langle\phi_{n}, E_{\Delta}^{j j} \phi_{n}\right\rangle+\left\langle\psi_{n}, E_{\Delta}^{i i} \psi_{n}\right\rangle\right) .
\end{aligned}
$$

The expectation on the right side of (7.22) can be bounded above by

$$
\mathbb{E}_{\Lambda}\left(\left|\operatorname{Tr}\left(P_{0}^{j i} E_{\Delta}^{i j}\right)\right|\right) \leq \frac{1}{2}\left\|P_{0}^{j i}\right\|_{1} \sup _{n} \mathbb{E}_{\Lambda}\left(\left\langle\phi_{n}, E_{\Delta}^{j j} \phi_{n}\right\rangle+\left\langle\psi_{n}, E_{\Delta}^{i i} \psi_{n}\right\rangle\right)
$$

We estimate the two inner products on the right side of (7.23) using the spectral averaging result Corollary 3.1, with $B$ equal to $u_{j}^{1 / 2}$ and $u_{i}^{1 / 2}$, respectively. Consequently, (7.20) is bounded above by

$$
M^{2}|| g\left\|_{\infty}|\Delta| \sum_{i, j}\right\| P_{0}^{j i} \|_{1}
$$

since $C_{0}=1$.

4. To evaluate the sum, we first consider those indices $i$ and $j$ for which $|i-j|<2$. Let $\chi_{i j}$ be the characteristic function for supp $\left(u_{i}+u_{j}\right)$. Then the contribution from these indices to the sum in (7.24) is

$$
\begin{aligned}
\sum_{|i-j|<2}\left\|P_{0}^{j i}\right\|_{1} & \leq\|u\|_{\infty}^{2} \sum_{|i-j|<2}\left\|\chi_{i j} P_{0} \chi_{i j}\right\|_{1} \\
& \leq C_{1} B|\Lambda||\operatorname{supp} u|,
\end{aligned}
$$

by Lemma 7.4, part (1). Next, in order to estimate the sum over the complimentary set of indices, we define the function $\chi_{i j}^{+}$to be the characteristic function for the set $\left\{x \in R^{2}|| x-i|<| x-j \mid\right\}$, and write $\chi_{i j}^{-} \equiv 1-\chi_{i j}^{+}$. Using the inequality

$$
\|A B\|_{1} \leq\|A\|_{H S}\|B\|_{H S}
$$

we obtain

$$
\left\|P_{0}^{j i}\right\|_{1} \leq\left\|u_{j}^{1 / 2} P_{0} \chi_{i j}^{+}\right\|_{H S}\left\|\chi_{i j}^{+} P_{0} u_{i}^{1 / 2}\right\|_{H S}+\left\|u_{j}^{1 / 2} P_{0} \chi_{i j}^{-}\right\|_{H S}\left\|\chi_{i j}^{-} P_{0} u_{i}^{1 / 2}\right\|_{H S} .
$$


If $|i-j| \geq 2$, condition (V1) on the support of $u_{j}$ implies that

$$
\begin{aligned}
\operatorname{dist}\left(\operatorname{supp} \chi_{i j}^{+}, \operatorname{supp} u_{j}\right) & \geq \frac{|i-j|}{2}-\frac{1}{\sqrt{2}} \\
& \geq a|i-j|,
\end{aligned}
$$

for some strictly positive constant $a$. A similar inequality holds for $\operatorname{dist}\left(\operatorname{supp} \chi_{i j}^{-}, \operatorname{supp} u_{i}\right)$. By part (2) of Lemma 7.4, one obtains from (7.27) and (7.28),

$$
\left\|P_{0}^{j i}\right\|_{1} \leq 2 C_{0}^{2} B|\operatorname{supp} u| e^{-a B|i-j|^{2} / 8} .
$$

from which it follows that

$$
\sum_{|i-j| \geq 2}\left\|P_{0}^{j i}\right\|_{1} \leq C_{2}|\operatorname{supp} u||\Lambda| .
$$

Combining (7.25) and (7.30) in (7.24), we obtain an upper bound for all $B$ large enough,

$$
\mathbb{E}_{\Lambda}\left(\operatorname{Tr} E_{\Delta}\right) \leq C_{W}[\operatorname{dist}(B, E)-\delta]^{-2} B\|g\|_{\infty}|\Delta||\Lambda|,
$$

where $C_{W}$ depends on $M,\|u\|_{\infty}$, and supp $u$. This proves the theorem.

7.5. Percolation Theory. The most interesting aspect of the proof of localization for the randomly perturbed Landau Hamiltonian is the use of classical bond percolation theory. This is used in order to prove the initial length-scale exponential decay estimate $[\mathrm{H} 1]\left(\gamma_{0}, \ell_{0}\right)$. We begin by giving a brief overview of percolation theory in two dimensions. These are standard results on percolation theory which can be found in [69] and [22].

Let $\mathbb{Z}^{2}$ be the square lattice (the length of the side plays no role in the calculations). A bond (edge) of $\mathbb{Z}^{2}$ is said to be occupied with probability $p, 0 \leq p \leq 1$, and empty with probability $1-p$. We are interested in the case when the bonds are independent. This is called Bernoulli bond percolation. The critical percolation probability $p_{c}$ is defined as follows. Let $P_{\infty}(p)$ be the probability that the origin belongs to an infinite, connected, cluster of occupied bonds. Then, we define

$$
p_{c} \equiv \inf \left\{p \mid P_{\infty}(p)>0\right\} .
$$

For 2-dimensional Bernoulli bond percolation, it is a major result that $p_{c}=1 / 2$. Hence if $p>p_{c}$, occupied bonds percolate to infinity. That is, we can find a connected cluster of occupied bonds running off to infinity with non-zero probability.

Of importance for us are the results concerning the existence of closed circuits of occupied bonds. Let $r_{n, \ell}$ be a rectangle in $\mathbb{Z}^{2}$ of width $\ell$ and length $n \ell$. Let $R_{n, \ell}$ be the probability that there is a crossing of $r_{n, \ell}$, the long way, by a connected path of occupied bonds. This probability is controlled by an exponential factor $m(p)$, which is strictly positive for $p<p_{c}$ and $m(p) \searrow 0$ as $p / p_{c}$. This factor measures the probability that the origin $0 \in \mathbb{Z}^{2}$ is connected to $x \in \mathbb{Z}^{2}$ by a path of occupied bonds

$$
P_{0 x}(p) \leq e^{-m(p)|x|}
$$

The basic result on the probability that a rectangle $r_{n, \ell}$ is crossed, the long way, by a cluster of occupied bonds is the following theorem.

THEOREM 7.6. For $p>p_{c}, R_{n, \ell} \geq 1-C_{0} n \ell e^{-m(1-p) \ell}$, for some constant $C_{0}$. 
We will relate the existence of an effective potential barrier, which prevents an electron from percolating to infinity, to the existence of annular regions, about any point in space, which contain a closed cluster of occupied sites. From Theorem 7.6, we can derive the probability that such closed clusters exist in annular regions.

Let us write $r_{\ell}$ for $r_{1, \ell}$, the square box of side length $\ell$. An annular region of width $\ell$, between two concentric boxes, is denoted by $a_{\ell} \equiv r_{3 \ell} \backslash r_{\ell}$. A closed circuit of occupied bonds in $a_{\ell}$ is a connected path of occupied bonds lying entirely within $a_{\ell}$. Using Theorem 7.6 and the $F G K$ inequality, one can compute the probability $A_{\ell}$ of a closed circuit of occupied bonds in $a_{\ell}$ for $p>p_{c}$.

Theorem 7.7. For any $p \in[0,1], A_{\ell} \geq\left[R_{3, \ell}(p)\right]^{4}$. In particular, if $p>$ $p_{c}, \exists 0<C_{0}<\infty$ as in Theorem 7.6, such that

$$
A_{\ell} \geq 1-12 C_{0} \ell e^{-m(1-p) \ell} .
$$

7.6. Exponential Decay Estimates from Percolation Theory. Some of the earlier work on randomly perturbed Landau Hamiltonians concentrated on the one-band approximation. Instead of studying the full Hamiltonian $H_{\omega}$ directly, one considers the restricted Hamiltonian $P_{0} H_{\omega} P_{0}$ on the subspace $P_{0} L^{2}\left(\mathbb{R}^{2}\right)$ (cf. $[\mathbf{4 0}$, 41]). In the situation when the random potential is bounded, this approximation is reasonable for large magnetic fields since the separation of the Landau bands is $\mathcal{O}(B)$. Hence, one expects the interband interaction to be small. We will prove that this is true. In this way, the one-band approximation will give us some insight into the nature of localization.

Formally, if one neglects the band interaction, the effective Hamiltonian for an electron at energy $E$, near the first Landau level $E_{0}(B)=B$, is $E \sim P_{0} H_{\omega} P_{0}=$ $B+P_{0} V_{\omega} P_{0} \sim B+V_{\omega}(x)$. We assume that the interaction terms, $P_{0} V_{\omega} Q_{0}$, and its adjoint, and the term coming from the other bands, $Q_{0} V_{\omega} Q_{0}$, are small. Consequently, in this approximation, the electron motion is along equipotential lines $V(x)+B-E=0$. Since the potential $V$ is random, it is natural to estimate the probability that these equipotential lines percolate through a given box. If there is no percolation, the (classical) electron will remain confined to bounded regions. One can expect that the interband interaction will not change this picture. We will first show how to reformulate our problem as a problem in bond percolation. We will then show that the Green's function decays exponentially in $x$ and $B$ through regions where $|V(x)+B-E|>a>0$.

Recall that $V_{\omega}(x)=\sum_{i \in \mathbb{Z}^{2}} \omega_{i} u(x-i)$, where the single-site potential $u \geq 0$ and has support inside a ball of radius $r_{u}<1 / \sqrt{2}$. We define $r_{u}$ to be the smallest radius such that supp $u \subset B\left(0, r_{u}\right)$. Consider a new square lattice $\Gamma \equiv e^{i \pi / 4} \sqrt{2} \mathbb{Z}^{2}$. The midpoint of each bond of $\Gamma$ is a site of $\mathbb{Z}^{2}$. We will denote by $b_{j}$ the bond of $\Gamma$ having $j \in \mathbb{Z}^{2}$ as it's midpoint. For definiteness, we assume $E \in\left(B, B+M_{0}\right)$. The other energy interval $\left(B-M_{0}, B\right)$ can be treated similarly.

Definition 7.8. The bond $b_{j}$ of $\Gamma$ is occupied if $\omega_{j}<(E-B) / 2$. The probability $\mathbb{P}\left\{\omega_{j}<E-B / 2\right\} \equiv p$ is the probability that $b_{j}$ is occupied ( $p$ is independent of $j$ by the iid assumption).

Let us assume that the bond $b_{j}$ is occupied and consider,

$$
\mathcal{R}_{j} \equiv\left\{x \mid \operatorname{dist}\left(x, b_{j}\right)<1 / \sqrt{2}-r_{u} \equiv r_{1}\right\} .
$$


Obviously, $\mathcal{R}_{j}$ does not intersect the support of the other single-site potentials centered on $\mathbb{Z}^{2} \backslash\{j\}$ so that $V(x)=\omega_{j} u(x-j) \forall x \in \mathcal{R}_{j}$. Then, if $b_{j}$ is occupied, one has $V(x)<(E-B) / 2 \forall x \in \mathcal{R}_{j}$ (recall that $(E-B) / 2>0$ ). We now assume that there is a closed circuit of occupied bonds $\mathcal{C} \equiv \cup_{j \in \gamma} b_{j}, \gamma \subset \mathbb{Z}^{2}$ (i.e. a connected union of occupied bonds). We call $\mathcal{R} \equiv \cup_{j \in \gamma} \mathcal{R}_{j}$ the closed ribbon associated with $\mathcal{C}$. For all $x \in \mathcal{R}$, we have $V(x)<(E-B) / 2$. If we take $a \equiv(E-B) / 2$, then

$$
V(x)+B-E<-a \forall x \in \mathcal{R} .
$$

The existence of a closed ribbon $\mathcal{R}$ so that $V$ satisfies condition (7.35) is a consequence of the existence of a closed circuit $\mathcal{C}$ in $\Gamma$ of occupied bonds. We saw in the previous section how to estimate the probability that $\mathcal{C}$ exists. We now apply the result of Theorem 7.7 to our reformulation of the equipotential line problem. First, we need a definition. For a subset $\mathcal{O} \subset \mathbb{R}^{2}$, the in-radius of $\mathcal{O}$ is defined to be $\sup \left\{R>0 \mid B_{R} \subset \mathcal{O}\right\}$, where $B_{R}$ denotes a ball of radius $R$. We will write Inrad $\mathcal{O}$ for the in-radius of $\mathcal{O}$.

On the lattice $\Gamma$, the probability that any bond is occupied is given by

$$
p=\int_{-M}^{a} g(\omega) d \omega
$$

so, under our assumptions on the density $g$, if $a>0$ then $p>p_{c}=1 / 2$, and we are above the critical percolation threshold $p_{c}=1 / 2$. Note that when $E=B, a=0$ so $p=1 / 2=p_{c}$, the critical probability. It follows from Theorem 7.7 that any annular region $a_{\ell} \equiv r_{3 \ell} \backslash r_{\ell}$ in $\Gamma$ of in-radius $\sqrt{2} \ell \equiv 1 / 2(3 \sqrt{2} \ell-\sqrt{2} \ell)$ and sides parallel to the bonds of $\Gamma$ contains a closed circuit of occupied bonds with probability given by (7.33). By the argument above, there is a ribbon $\mathcal{R}$ associated with $\mathcal{C}$ in $a_{\ell}$ whose properties we summarize in the next proposition.

Proposition 7.1. Assume (V1) and (V2) and suppose that supp $u \subset B_{r_{u}}(0)$. Let $\ell>\sqrt{2}, E \in \sigma_{0} \backslash\{B\}$, and $a>0$. Then for $m(1-p)$ and $C_{0}$ as in Theorem 7.8, there exists a ribbon $\mathcal{R}$ satisfying

$$
\begin{aligned}
& \operatorname{dist}\left(\mathcal{R}, \partial r_{3 \ell}\right), \operatorname{dist}\left(\mathcal{R}, \partial r_{\ell}\right) \geq 1 / \sqrt{2}+r_{u} ; \\
& \mathcal{R} \subset a_{\ell},
\end{aligned}
$$

and such that

$$
V(x)+B-E<-a, \forall x \in \mathcal{R},
$$

with a probability larger than

$$
1-12 C_{0} \ell e^{-m(1-p) \ell}
$$

where

$$
p \equiv \int_{-M}^{a} g(\omega) d \omega
$$

It is important to note that the probability $p$ defined in (7.40) is a function of $a$. In the applications, the constant $0<a=(E-B) / 2$, for $E>B$. As $E$ approaches the Landau level $B, a \rightarrow 0$ and $p \rightarrow p_{c}$, so it is important to have a lower bound 
on the effective mass $m(p(a))$, defined in (7.32), in order to obtain both the region of localization to within $O(\log B / B)$ of the Landau levels, and good exponential bounds on the eigenfunctions. We refer to [64] for a detailed discussion of this question.

The ribbon $\mathcal{R}$ i described in Proposition 7.1 provides an effective barrier for the equipotential lines along which a classical electron at energy $E$ would move. The addition of quantum effects results in exponential decay of the resolvent of the Hamiltonian at energy $E$ through the ribbon region. We now show how to prove such an estimate. This will lead to our verification of $[\mathrm{H} 1]\left(\gamma_{0}, \ell_{0}\right)$. By the geometric resolvent equation, we will show that it suffices to consider the following ideal situation, where for some $a>0$,

$$
V(x)+B-E<-a, \forall x \in \mathbb{R}^{2},
$$

or, alternately,

$$
V(x)+B-E>a, \forall x \in \mathbb{R}^{2} .
$$

A condition such as (7.41) with $E>B$ is satisfied, with a probability given in Proposition 7.1, by a smoothing of the potential $V_{\mathcal{R}}$ defined as

$$
V_{\mathcal{R}}(x)= \begin{cases}V(x) & x \in \mathcal{R} \\ 0 & x \in \mathbb{R}^{2} \backslash \mathcal{R} .\end{cases}
$$

Here we obtain decay estimates on the model Hamiltonian

$$
H_{\mathcal{R}} \equiv H_{A}+V_{\mathcal{R}},
$$

with $V_{\mathcal{R}}$ having compact support with nonempty interior and satisfying (7.41) or (7.42).

As is often the case, we will derive exponential decay estimates through the introduction of analytic families of operators associated with $H_{\omega}$, and the projectors $P_{0}$ and $Q_{0}$. We will obtain a priori bounds on these operators using condition (7.41) or (7.42). The type of argument used in the proof of the next lemma is similar to that used in the proof of the Combes-Thomas estimate given in [8], so we will just sketch it.

Let $\mathcal{O}$ be an open, bounded, connected set in $R^{2}$ with smooth boundary and define $\rho(x)=\operatorname{dist}(x, \mathcal{O})$. Let $\eta \in C_{0}^{\infty}\left(R^{2}\right)$ with $\eta>0$ and supp $\eta \subset B_{1}(0)$. For any $\epsilon>0$, define $\eta_{\epsilon}(x)=\eta(x / \epsilon)$. We consider the smoothed distance function $\rho_{\epsilon}(x) \equiv\left(\eta_{\epsilon} \star \rho\right)(x) ; \operatorname{supp} \rho_{\epsilon} \subset \mathbb{R}^{2} \backslash\left\{x \mid\right.$ dist $\left.\left(x, \mathcal{O}^{c}\right)<\epsilon\right\}$. We fix $\epsilon>0$ small and write $\rho$ for $\rho_{\epsilon}$ below for simplicity. We have $\|\nabla \rho\|_{\infty}<C_{0} / \epsilon$ and $\|\Delta \rho\|_{\infty}<C_{1} / \epsilon^{2}$, for constants $C_{0}, C_{1}>0$ depending only on $\eta$ and $\mathcal{O}$. This $\epsilon$ will play no role in the analysis below and, consequently, we absorb it into the constants $C_{0}$ and $C_{1}$. We consider one-parameter families of operators defined for $\alpha \in \mathbb{R}$ as

$$
\begin{aligned}
H_{A}(\alpha) & \equiv e^{i \alpha \rho} H_{A} e^{-i \alpha \rho} \\
H(\alpha) & \equiv H_{A}(\alpha)+V \\
P(\alpha) & \equiv e^{i \alpha \rho} P e^{-i \alpha \rho}, \text { etc.. }
\end{aligned}
$$

Here, we write $P$ for the projector $P_{0}$ and $Q \equiv 1-P$. For $\alpha \in R$, these families are unitarily equivalent with the $\alpha=0$ operators.

Lemma 7.9. The family $H(\alpha), \alpha \in \mathbb{R}$, has an analytic continuation into the strip

$$
S \equiv\left\{\alpha \in \mathbb{C}|| \operatorname{Im} \alpha \mid<\eta_{\rho} B^{1 / 2},\right\}
$$


as a type $A$ analytic family with domain $D(H)$. The positive constant $\eta_{\rho}$ depends only on the distance function $\rho$. Furthermore, in this strip $S$, one has $P(\alpha)^{2}=$ $P(\alpha)$, and for some constant $C_{1}$ independent of $\alpha$,

$$
\|P(\alpha)\|<C_{1}
$$

and

$$
\left\|Q(\alpha)\left(H_{A}(\alpha)-z\right)^{-1}\right\|<C_{1} B^{-1} \text {, if } \operatorname{dist}(z, B) \leq B .
$$

Sketch of the Proof. For $\alpha \in \mathbb{R}$, one has

$$
\begin{aligned}
H_{A}(\alpha) & =(-i \nabla-\alpha \nabla \rho-A)^{2} \\
& =H_{A}-\alpha[\nabla \rho \cdot(p-A)+(p-A) \cdot \nabla \rho]+\alpha^{2}|\nabla \rho|^{2} \\
& =H_{A}+\alpha^{2}|\nabla \rho|^{2}+i \alpha \Delta \rho-2 \alpha \nabla \rho \cdot(p-A) .
\end{aligned}
$$

We first show that $H_{A}(\alpha)$ is a type $A$ analytic family on the strip $S$. For this, it suffices to show that

$$
\left\{\alpha^{2}|\nabla \rho|^{2}+i \alpha \Delta \rho-2 \alpha \nabla \rho \cdot(p-A)\right\}\left(H_{A}-z\right)^{-1},
$$

has norm less than 1 for some $z \notin \sigma\left(H_{A}\right)$ and $|\operatorname{Im} \alpha|<\eta_{\rho} B^{1 / 2}$ (cf [81], in particular, Theorem IV.1.1 and Chapter VII.2). We now proceed to bound each term for $z \in C(B) \equiv\{z|| z-B \mid=B\}$, circle of radius $B$ centered at $B$. Since type $A$ analyticity is stable under bounded perturbations, it follows that $H(\alpha)$ is a type $A$ analytic family of operators on $S$. For the projectors, we use the integral representation

$$
P(\alpha)=\frac{-1}{2 \pi i} \int_{C(B)}\left(H_{A}(\alpha)-z\right)^{-1} d z
$$

Theorem 7.10. Assume that $(V, E, B)$ satisfy (7.41) or (7.42) for some $a>0$ and $E \in \sigma_{0} \backslash\{B\}$. Furthermore, assume that supp $V$ is compact with non-empty interior. There exists constants $C_{2} \leq \eta_{\rho}, C_{3}$, and $B_{1}$, depending only on $M_{0} \equiv$ $\|V\|_{\infty},\|\nabla \rho\|_{\infty}$, and $\|\nabla V\|_{\infty}$, such that if we define $\gamma \equiv C_{2} \min \left\{B^{1 / 2}, a B\right\}$, and $u$ is a solution of

$$
\left(H_{A}+V-z\right) u=v, z \equiv E+i \epsilon, \epsilon>0, E>0,
$$

for some $v \in D\left(e^{\gamma \rho}\right)$, then for $B>B_{1}, \forall \alpha \in \mathbb{C},|\operatorname{Im} \alpha|<\gamma$, we have

$$
\begin{gathered}
u \in D\left(e^{i \rho \alpha}\right), \\
\left\|e^{i \alpha \rho} P u\right\| \leq C_{3} a^{-1}\left\|e^{i \alpha \rho} v\right\|,
\end{gathered}
$$

and

$$
\left\|e^{i \alpha \rho} Q u\right\| \leq C_{3} B^{-1}\left\|e^{i \alpha \rho} v\right\| .
$$

Proof. Let $v(\alpha)=e^{i \alpha \rho} v$, so that $v(\alpha)$ is analytic in the strip $|\operatorname{Im}(\alpha)|<\gamma$. Let $u(\alpha)=e^{i \alpha \rho} u, \alpha \in \mathbb{R}$, i.e.,

$$
u(\alpha)=(H(\alpha)-z)^{-1} v(\alpha) \equiv\left((H-z)^{-1} v\right)(\alpha),
$$

with $H \equiv H_{A}+V$, as above. Since $V$ is $H_{A}$-compact by assumption, $H$ has point spectrum, and according to Lemma 7.9 and standard arguments, $H(\alpha)$ has real spectrum independent of $\alpha$ in the strip $S$ defined in (7.48). Then, $u(\alpha)$ has an analytic continuation in $|\operatorname{Im}(\alpha)|<\gamma$, which proves (7.54). Furthermore, this 
continuation satisfies (7.53) in the whole strip $S$, i.e. $(H(\alpha)-z) u(\alpha)=v(\alpha)$, for all $\alpha \in S$. Projecting this equation along $P(\alpha)$ gives

$$
(B+V-z)(P u)(\alpha)=(P v)(\alpha)+([Q V P-P V Q] u)(\alpha),
$$

where $(P u)(\alpha) \equiv P(\alpha) u(\alpha)$, etc. Taking the scalar product of $(7.58)$ with $(P u)(\alpha)$ results in the inequality,

$$
\begin{aligned}
a\|(P u)(\alpha)\|^{2} & \leq\|(P u)(\alpha)\|\|(P v)(\alpha)\| \\
& +\left\{\left\|\left(P^{*} Q\right)(\alpha)\right\|\|(Q V P)(\alpha)\|\right\}\|(P u)(\alpha)\|^{2} \\
& +\|(P V Q)(\alpha)\|\|(P u)(\alpha)\|\|(Q u)(\alpha)\| .
\end{aligned}
$$

One can prove that for $B$ large enough,

$$
\|(Q V P)(\alpha)\| \leq C_{4} B^{-1 / 2}
$$

and

$$
\left\|\left(P^{*} Q\right)(\alpha)\right\| \leq C_{5}|\operatorname{Im} \alpha| B^{-1 / 2} .
$$

With these estimates, we obtain from (7.59),

$$
\begin{aligned}
\left(a-C_{6} \gamma B^{-1}\right)\|(P u)(\alpha)\|^{2} & \leq\|(P u)(\alpha)\|\|(P v)(\alpha)\| \\
& +C_{7} B^{-1 / 2}\|(P u)(\alpha)\|\|(Q u)(\alpha)\|,
\end{aligned}
$$

where the constants $C_{6}$ and $C_{7}$ depend only on $\|V\|_{\infty},\|\nabla V\|_{\infty}$, and $\|\nabla \rho\|_{\infty}$. To estimate $\|(Q u)(\alpha)\|$, it follows from the resolvent equation and (7.57) that (7.63)

$$
\begin{aligned}
\|(Q u)(\alpha)\| & \leq\left\|\left(Q\left(H_{A}-z\right)^{-1} v\right)(\alpha)\right\|+\left\|\left\{Q\left(H_{A}-z\right)^{-1} Q V(Q+P) u\right\}(\alpha)\right\| \\
& \leq C_{1} B^{-1}\|v(\alpha)\|+C_{1} B^{-1} M_{0}\|(Q u)(\alpha)\| \\
& +C_{1} B^{-1} M_{0}\|(Q V P u)(\alpha)\|,
\end{aligned}
$$

with $M_{0} \equiv\|V\|_{\infty}<\infty$. Using an estimate on $Q V P$ similar to (7.60), and taking $B>2 M_{0} C_{1}$, we obtain,

$$
\|(Q u)(\alpha)\| \leq 2 C_{1} B^{-1}\|v(\alpha)\|+C_{8} B^{-3 / 2}\|(P u)(\alpha)\|,
$$

where $C_{8} \equiv 2 M_{0} C_{1} C_{2}$. Substituting (7.64) into (7.62), we obtain

$$
\left(a-C_{6} \gamma B^{-1}-C_{7} C_{8} B^{-2}\right)\|(P u)(\alpha)\| \leq\left(C_{1}+2 C_{1} C_{7} B^{-3 / 2}\right)\|v(\alpha)\| .
$$

This proves (7.55) for $B$ large enough. Inserting (7.55) into (7.64) yields (7.56).

Corollary 7.1. Let $\mathcal{O}$ be an open, connected, bounded subset of $\mathbb{R}^{2}$ with smooth boundary, and suppose $\mathcal{E} \subset \mathbb{R}^{2} \backslash \mathcal{O}$. Let $E \in \sigma_{0} \backslash\{B\}$ and assume that $(B, E, V)$ satisfy $(7.41)$ or (7.42) for some $a>0$. Let $\chi_{X}, X=\mathcal{O}$ and $\mathcal{E}$, be bounded functions with support in $X$ and s.t. $\left\|\chi_{X}\right\|_{\infty} \leq 1$. Then,

$$
\sup _{\epsilon \neq 0}\left\|\chi_{\mathcal{E}}\left(H_{A}+V-E-i \epsilon\right)^{-1} \chi_{\mathcal{O}}\right\| \leq C \max \left\{a^{-1}, B^{-1}\right\} e^{-\gamma d},
$$

where $C$ and $\gamma$ are as in Theorem 7.10 and $d \equiv \operatorname{dist}(\mathcal{O}, \mathcal{E})$. 
Proof. This is an immediate consequence of Theorem 7.10 We set $\rho(x) \equiv \operatorname{dist}(x, \mathcal{O})$ and choose $v \equiv \chi_{\mathcal{O}} v$. Then, $e^{i \alpha \rho} v=v, \forall \alpha \in \mathbb{C}$. For $u$ a solution of $\left(H_{A}+V-\right.$ $E-i \epsilon) u=\chi_{\mathcal{O}} v$, one has $\forall \alpha \in \mathbb{C},|\operatorname{Im} \alpha|<\gamma$,

$$
\begin{gathered}
\left\|\chi_{\mathcal{E}}\left(H_{A}+V-E-i \epsilon\right)^{-1} \chi_{\mathcal{O}} v\right\|=\left\|\chi_{\mathcal{E}}(P+Q) u\right\| \\
\leq e^{-d(\operatorname{Im} \alpha)}\left\{\left\|e^{-i \alpha \rho} P u\right\|+\left\|e^{-i \alpha \rho} Q u\right\|\right\} \\
\leq e^{-d(\operatorname{Im} \alpha)} C \max \left\{a^{-1}, B^{-1}\right\}\|v\|,
\end{gathered}
$$

by Theorem 7.17 Taking $\operatorname{Im} \alpha \rightarrow \gamma$, we obtain (7.66).

7.7. Verification of Hypothesis $[\mathbf{H} 1]\left(\gamma_{0}, \ell_{0}\right)$. It remains to verify the initial length-scale hypothesis. The proof of localization then follows the lines as for Schrödinger operators as given in chapter 6 , but requires the bootstrap MSA of Germinet and Klein $[62,63]$. In particular, we see from (7.67) that the exponential decay of the localized resolvent at initial length scale $\ell_{0}$ is approximately $\sim C_{1} \min \left(a B, B^{1 / 2}\right) / \ell_{0}$. From this we see that $\ell_{0}$ cannot be too big relative to $B$ and $a$. On the other hand, the MSA requires the initial length scale to be large enough. These two opposing demands require a more refined multiscale analysis than the one presented in chapter 8 and we refer to $[\mathbf{6 2}, \mathbf{6 3}]$. The underlying ideas, however, are similar.

Proposition 7.2. Let $\chi_{2}$ be any function, $\left\|\chi_{2}\right\|_{\infty} \leq 1$, supported on $\Lambda_{\ell} \cap$ ExtR, where $E x t \mathcal{R} \equiv\left\{x \in \mathbb{R}^{2} \mid \lambda x \notin \mathcal{R} \forall \lambda \geq 1\right\}$, so that, in particular, supp $\chi_{2} \cap$ $\mathcal{R}=\emptyset$. For any $E \in \sigma_{0} \backslash\{B\}, \delta>0, \epsilon>0$, and $a>0$, we have

$$
\begin{aligned}
\sup _{\epsilon \neq 0}\left\|\chi_{2} R_{\Lambda_{\ell}}(E+i \epsilon) \chi_{\ell / 3}\right\| \leq & C e^{-\gamma d} \max \left\{a^{-1}, B^{-1}\right\} \cdot \max \left\{\delta^{-1},\right. \\
& \left.\left(2 M_{0}+|E|\right) \delta^{-2}\right\},
\end{aligned}
$$

where $C$ depends on $C_{3}$ of Theorem 7.10, the constants $\chi_{2}$ and $\gamma$ are defined in Theorem 7.10 , and $d \equiv\left(r_{1}-3 \epsilon\right) / 2\left(r_{1} \equiv\right.$ inrad $\left.\mathcal{R}\right)$, with a probability larger than

$$
1-\left\{C \ell e^{-m \ell}+C_{W}[\operatorname{dist}(E, B)-\delta]^{-2}\|g\|_{\infty} \delta B \ell^{2}\right\} .
$$

In particular, for $\chi_{\ell, \delta}$ defined above and $E \in \sigma_{0}$ with $a=(E-B) / 2=\mathcal{O}\left(B^{-1+\sigma}\right)$, any $\sigma>0$, we have that for any $\ell_{0}>\sqrt{2}$ and large enough, and any $\xi>4, \exists B\left(\ell_{0}\right)>0$ such that $\forall B>B\left(\ell_{0}\right),[\mathrm{H} 1]\left(\gamma_{0}, \ell_{0}\right)$ holds for some $\gamma_{0}>$ $\gamma d / 4 \ell_{0}>0$, so that $\gamma_{0}=\mathcal{O}\left\{\min \left(B^{1 / 2}, B^{\sigma}\right)\right\}$.

\section{Proof.}

1. By Proposition 7.1, there exists a constant $B_{0}$ such that $B>B_{0}$ implies there exists a ribbon $\mathcal{R} \subset \Lambda_{\ell} \backslash \Lambda_{\ell / 3}$ (with a probability given by (7.39) satisfying

$$
\operatorname{dist}\left(\mathcal{R}, \partial \Lambda_{\ell}\right) \text {, and } \operatorname{dist}\left(\mathcal{R}, \partial \Lambda_{\ell / 3}\right)>1 / \sqrt{2}+r_{u}>0,
$$

and

$$
r_{1} \equiv \operatorname{inrad\mathcal {R}}>2\left(1 / \sqrt{2}-r_{u}\right)
$$

and such that

$$
V(x)+B-E>-a \forall x \in \mathcal{R}, a=E-B / 2 .
$$


We assume $E>B$; similar arguments hold for $E<B$. For any $\epsilon>0,3 \epsilon \ll r_{1}$, define the border of $\mathcal{R}$ by

$$
\mathcal{R}_{\epsilon} \equiv\{x \in \mathcal{R} \mid \operatorname{dist}(x, \partial \mathcal{R})<\epsilon\} .
$$

Then $\mathcal{R}_{\epsilon} \equiv \mathcal{R}_{\epsilon}^{+} \cup \mathcal{R}_{\epsilon}^{-}$, where $\mathcal{R}_{\epsilon}^{ \pm}$are two disjoint, connected subsets of $\mathcal{R}$. Let $\mathcal{C} \equiv\left\{x \in \mathcal{R} \mid \operatorname{dist}\left(x, \mathcal{R}_{\epsilon}^{+}\right)=\operatorname{dist}\left(x, \mathcal{R}_{\epsilon}^{-}\right)\right\} ; \mathcal{C}$ is a closed, connected path in $\mathcal{R}$. Let $\mathcal{C}_{\epsilon} \equiv\{x \in \mathcal{R} \mid$ dist $(x, \mathcal{C})<\epsilon / 2\} \subset \mathcal{R}$, so that inrad $\mathcal{C}_{\epsilon}=\epsilon$ and

$$
\operatorname{dist}\left(\mathcal{C}_{\epsilon}, \mathcal{R}_{\epsilon}^{ \pm}\right) \geq\left(r_{1}-3 \epsilon\right) / 2 \text {. }
$$

This is strictly positive. Because of this, we can adjust $\mathcal{C}_{\epsilon}$ so that $\partial \mathcal{C}_{\epsilon}$ is smooth. We need two, $C^{2}$, positive cut-off functions. Let $\chi_{\mathcal{R}}>0$ satisfy $\chi_{\mathcal{R}} \mid \mathcal{C}_{\epsilon}=1$ and $\operatorname{supp}\left|\nabla \chi_{\mathcal{R}}\right| \subset \mathcal{R}_{\epsilon}$. Let $\chi_{1}$ satisfy $\chi_{1} \mid \Lambda_{\ell / 3}=1$ and $\operatorname{supp}\left|\nabla \chi_{1}\right| \subset \mathcal{C}_{\epsilon}$. By simple commutation, we have (with $\chi_{2}$ as in the proposition),

$$
\begin{aligned}
\chi_{2} R_{\Lambda_{\ell}}(E+i \epsilon) \chi_{\ell / 3} & =\chi_{2} R_{\Lambda_{\ell}} \chi_{1} \chi_{\ell / 3} \\
& =\chi_{2} R_{\Lambda_{\ell}} W\left(\chi_{1}\right) R_{\Lambda_{\ell}} \chi_{\ell / 3} \\
& =\chi_{2} R_{\Lambda_{\ell}} \chi_{\mathcal{R}} W\left(\chi_{1}\right) R_{\Lambda_{\ell}} \chi_{\ell / 3} .
\end{aligned}
$$

Next, denote by $R_{\mathcal{R}}$ the resolvent of $H_{\mathcal{R}}$ defined in (7.44). The GRE relating $R_{\Lambda_{\ell}}$ and $R_{\mathcal{R}}$ is

$$
R_{\Lambda_{\ell}} \chi_{\mathcal{R}}=\chi_{\mathcal{R}} R_{\mathcal{R}}+R_{\Lambda_{\ell}} W\left(\chi_{\mathcal{R}}\right) R_{\mathcal{R}} .
$$

Substituting (7.74) into (7.73) and noting that $\chi_{2} \chi_{\mathcal{R}}=0$, we obtain

$$
\chi_{2} R_{\Lambda_{\ell}} \chi_{\ell / 3}=\chi_{2} R_{\Lambda_{\ell}} W\left(\chi_{\mathcal{R}}\right) R_{\mathcal{R}} W\left(\chi_{1}\right) R_{\Lambda_{\ell}} \chi_{\ell / 3} .
$$

Note that from (7.72) and the choice of $\chi_{\mathcal{R}}$ and $\chi_{1}$, we obtain that

$$
\operatorname{dist}\left(\operatorname{supp} W\left(\chi_{\mathcal{R}}\right), \operatorname{supp} W\left(\chi_{1}\right)\right) \geq\left(r_{1}-3 \epsilon\right) / 2 \text {. }
$$

We apply Wegner's estimate, Theorem 7.4 to control the two $R_{\Lambda_{\ell}}$ factors in (7.75), and the decay estimate, Corollary 7.1, to control the factor $R_{\mathcal{R}}$, which is possible due to the localization of $W\left(\chi_{\mathcal{R}}\right)$ and $W\left(\chi_{1}\right)$ and (7.76).

2. To estimate the $R_{\mathcal{R}}(E+i \epsilon)$ contribution, we use Corollary 7.1 with $\mathcal{O} \equiv \mathcal{C}_{\epsilon}$ and $\mathcal{E}=\mathcal{R}_{\epsilon}$. Let $\chi_{X}, X=\mathcal{O}$ and $\mathcal{E}$, be a characteristic function on these sets. Then $W\left(\chi_{\mathcal{R}}\right) \chi_{\mathcal{E}}=W\left(\chi_{\mathcal{R}}\right)$ and $\chi_{\mathcal{O}} W\left(\chi_{1}\right)=W\left(\chi_{1}\right)$. Inserting these localization functions into (7.75), we obtain from Corollary 7.1,

$$
\left\|\chi_{\mathcal{E}} R_{\mathcal{R}}(E+i \epsilon) \chi_{\mathcal{O}}\right\| \leq C \max \left\{a^{-1}, B^{-1}\right\} e^{-\gamma d},
$$

with probability larger than

$$
1-C \ell e^{-m \ell},
$$

for some $m=m(1-p)>0$ (see (7.39) and $0<C<\infty$. The factor $d$ satisfies

$$
d \geq\left(r_{1}-3 \epsilon\right) / 2
$$

where $r_{1} \equiv$ inrad $\mathcal{R}$ as in (7.36).

3. Next, we turn to

$$
W\left(\chi_{1}\right) R_{\ell}(E+i \epsilon) \chi_{\ell / 3},
$$

and

$$
\chi_{2} R_{\ell}(E+i \epsilon) W\left(\chi_{\mathcal{R}}\right),
$$


where we write $R_{\ell}$ for $R_{\Lambda_{\ell}}$ for short. We will bound $R_{\ell}$ by Wegner's estimate and the terms (7.80)-(7.81) via Proposition 7.2. From Theorem 7.4, we have for any $\delta>0$

$$
\left\|R_{\ell}(E+i \epsilon)\right\|<\delta^{-1}
$$

with probability larger than

$$
1-C_{W}[\operatorname{dist}(E, B)-\delta]^{-2}\|g\|_{\infty} \delta B \ell^{2} .
$$

From Proposition 7.2, both (7.80) and (7.81) are bounded above by

$$
C_{4} \max \left\{\delta^{-1 / 2},(2 M+|E|)^{1 / 2} \delta^{-1}\right\},
$$

with probability at least (7.39). The constant $C_{4}$ depends on $\partial^{\alpha} \chi_{1}$, for $|\alpha|=0,1,2$. 4. Using the estimate $P(A \cap B) \geq P(A)+P(B)-1$, and (7.75)-(7.77), (7.77), and (7.82)-(7.83), we find

$$
\begin{aligned}
\left\|\chi_{2} R_{\ell}(E+i \epsilon) \chi_{\ell / 3}\right\| \leq & 2 C \max \left\{a^{-1}, B^{-1}\right\} \cdot \max \left\{\delta^{-1},\right. \\
& \left.\left(2 M_{0}+|E|\right) \delta^{-2}\right\} \cdot e^{-\gamma d},
\end{aligned}
$$

with probability at least

$$
1-\left\{C \ell e^{-m \ell}+C_{W}[\operatorname{dist}(E, B)-\delta]^{-2}\|g\|_{\infty} \delta B \ell^{2}\right\} .
$$

This proves the first part of the proposition.

5. To estimate $W\left(\chi_{\ell, \delta}\right) R_{\ell} \chi_{\ell / 3}$, we again use Proposition 7.2 which gives

$$
\begin{aligned}
\left\|\chi_{2}(p-A)_{i} R_{\ell} \chi_{\ell / 3}\right\|^{2} & \leq\left\|\chi_{2} R_{\ell} \chi_{\ell / 3}\right\|+(2 M+|E|)\left\|\chi_{2} R_{\ell} \chi_{\ell / 3}\right\|^{2} \\
& +2 \max _{i=1,2}\left\|\left(\partial_{i} \chi_{2}\right) R_{\ell} \chi_{\ell / 3}\right\|\left\|\chi_{2}(p-A)_{i} R_{\ell} \chi_{\ell / 3}\right\| .
\end{aligned}
$$

Since $\partial_{i} \chi_{2}$ satisfies the same condition as $\chi_{2}$, the factor $\left\|\left(\partial_{i} \chi_{2}\right) R_{\ell} \chi_{\ell / 3}\right\|$ in $(7.87)$ satisfies the estimate (7.85) with possibly a different constant. Solving the quadratic inequality (7.87), we obtain

$$
\begin{aligned}
\left\|\chi_{2}(p-A)_{i} R_{\ell} \chi_{\ell / 3}\right\| \leq & \max _{i=1,2}\left\{\left\|\left(\partial_{i} \chi_{2}\right) R_{\ell} \chi_{\ell / 3}\right\|\right. \\
& +\left[\left\|\left(\partial_{i} \chi_{2}\right) R_{\ell} \chi_{\ell / 3}\right\|^{2}+\left(\left\|\chi_{2} R_{\ell} \chi_{\ell / 3}\right\|\right.\right. \\
& \left.\left.\left.+\left(2 M_{0}+|E|\right)\left\|\chi_{2} R_{\ell} \chi_{\ell / 3}\right\|^{2}\right)\right]^{1 / 2}\right\}
\end{aligned}
$$

which can be estimated as in (7.85). Finally, we write

$$
\left\|W\left(\chi_{\ell, \delta}\right) R_{\ell} \chi_{\ell / 3}\right\| \leq\left\|\left(\Delta \chi_{\ell, \delta}\right) R_{\ell} \chi_{\ell / 3}\right\|+2 \sum_{j=1}^{2}\left\|\left(\partial_{j} \chi_{\ell, \delta}\right)(p-A)_{j} R_{\ell} \chi_{\ell / 3}\right\|
$$

which can be estimated from (7.77) with $\chi_{2} \equiv \Delta \chi_{\ell, \delta}$ and (7.88) with $\chi_{2} \equiv\left(\partial_{j} \chi_{\ell, \delta}\right)$. 6. We now show that for any $\ell_{0}$ large enough, $\exists B_{0} \equiv B_{0}\left(\ell_{0}\right)$ such that for all $B>B_{0}$, condition $[H 1]\left(\ell_{0}, \gamma_{0}\right)$ is satisfied with $\gamma_{0}=\mathcal{O}\left\{\min \left(B^{1 / 2}, B^{\sigma}\right)\right\}$. We take $E \in\left[B-M_{0}, B-\mathcal{O}\left(B^{-1}\right)\right] \cup I_{0}(B) \cap \sigma_{0}$ and $a=(E-B) / 2=\mathcal{O}\left(B^{-1+\sigma}\right)$, for any $\sigma>0$. First, we require that (7.66) be bounded above by $e^{-\gamma d / 2}$. This leads to the condition

$$
C \delta^{-2} B^{2-\sigma} e^{-\gamma d} \leq e^{-\gamma d / 2},
$$


where $\gamma=C_{2} \min \left\{B^{1 / 2}, B^{\sigma}\right\}$. This condition implies that we must choose $\delta$ in (7.90) to satisfy

$$
\delta>B^{1-(\sigma / 2)} e^{-\gamma d / 4} .
$$

If we now define $\gamma_{0} \equiv \gamma d / 4 \ell_{0}$, we find that

$$
\left\|W\left(\chi_{\ell, \delta}\right) R_{\ell} \chi_{\ell / 3}\right\| \leq e^{-\gamma_{0} \ell_{0}} .
$$

Next, in order to obtain the probability estimate (5.28), it follows from (7.67) that we must require that

$$
C \ell e^{-m \ell_{0}}+C_{2} B^{3-2 \sigma} \delta \ell_{0}^{2} \leq \ell_{0}^{-\xi},
$$

or, for all $\ell_{0}$ large,

$$
C_{3} B^{3-2 \sigma} \delta \ell_{0}^{2} \leq \ell_{0}^{-\xi},
$$

for some $\xi>4$. We can choose $\delta$ so that both conditions (7.90) and (7.94) are satisfied provided the condition

$$
\ell_{0}^{\xi+2}<B^{3 / 2-(5 / 2) \sigma} e^{\gamma d / 4},
$$

is satisfied for some $\xi>4$. It is clear from the definition of $\gamma$, that for any $\ell_{0}$, there exists a $B_{0} \equiv B_{0}\left(\ell_{0}\right)$ such that condition (5.28) is satisfied for all $B>B_{0}$. We remind the reader again that $m$ is a function of $a=(E-B) / 2$, so this must be kept in mind while making these estimates. This completes the proof of the theorem.

\section{Fixed-Energy Multiscale Analysis}

8.1. Introduction. In this chapter, we complete the discussion of chapter 5 on the multiscale analysis (MSA) by giving the proof of Lemma 5.4. We introduced two hypotheses on the finite-volume Hamiltonians, called [H1] $\left(\gamma_{0}, \ell_{0}\right)$ and the Wegner estimate $[\mathrm{W}]$, in chapter 5 . We show in this chapter that these two hypotheses, $[\mathrm{H} 1]\left(\gamma_{0}, \ell_{0}\right)$ and $[\mathrm{W}]$, allow us to prove the hypotheses [H1] and [H2] of chapter 5. Hypotheses [H1] and [H2] are required for Theorems 5.3 and 5.6. As a result of the proofs in this chapter, we will have shown that these two hypotheses $[\mathrm{H} 1]\left(\gamma_{0}, \ell_{0}\right)$ and $[\mathrm{W}]$ are the starting points for deriving the almost sure exponential decay of the infinite-volume Green's function. We saw in chapter 3 that this estimate on the resolvent is essential for eliminating the continuous singular spectrum almost surely. The proof given here is a simplified and modified version of the multiscale analysis for lattice models developed in the work of Fröhlich and Spencer [56], Spencer [120], and von Dreifus and Klein [132]. A summary of this method for lattice models is given in the book of Carmona and Lacroix [21]. The proof for random operators on $\mathbb{R}^{d}$ requires the use of geometric methods common in this book. The MSA used here is a fixed-energy MSA. There are other methods, pioneered by von Dreifus and Klein [132] in which all energies in a fixed interval are treated simultaneously. In this method, the techniques of chapter 3 are replaced by an analysis of the generalized eigenfunctions. This is discussed in the next section. The energy-interval method for Schrödinger operators on $L^{2}\left(\mathbb{R}^{d}\right)$ is presented in [62]. Let us first recall Lemma 5.4. 
Lemma 8.1. Let $\gamma_{0}>0$ and assume $[W]$. There exists a minimum length scale $l^{*}=l^{*}\left(\gamma_{0}, q, d, C_{W}\right)$ such that if $[H 1]\left(\gamma_{0}, l_{0}\right)$ holds for $l_{0}>l^{*}$, then for $l_{k} \equiv l_{0}^{(3 / 2)^{k}}$, and for some $\xi>2 d$, there exists a finite constant $\kappa>0$ such that for each $k$

$$
\mathbb{P}\left\{\sup _{\epsilon>0}\left\|W_{l_{k}} R_{l_{k}}(E+i \epsilon) \chi_{l_{k} / 3}\right\| \leq e^{-\kappa \gamma_{0} l_{k}}\right\} \geq 1-l_{k}^{-\xi} .
$$

This lemma shows that the two assumptions $[\mathrm{H} 1]\left(\gamma_{0}, \ell_{0}\right)$ and $[\mathrm{W}]$ are sufficient to prove the hypotheses of Theorem 5.3. Theorem 5.3 is the almost sure boundedness of the boundary-value of $\left\|\left(H_{\omega}-E-i 0^{+}\right)^{-1} c\right\|$, for suitable functions of compact support $c$.

8.2. Fixed Energy vs. Energy Interval Hypotheses. As has been discussed in previous chapters, we take the fixed energy approach to proving localization. Another approach, introduced in Fröhlich, Spencer, Martinelli, and Scoppola [55], and simplified in the work of von Dreifus and Klein [132], considers working in a fixed-energy interval. The technical aspects of these two approaches differ in two respects. First, the energy interval approach does not use the results on the perturbation of singular spectra presented in chapter 3. Instead, the approach uses the result that the spectrum of a Hamiltonian is characterized by the existence of polynomially-bounded solutions to the eigenvalue equation $H_{\omega} \psi_{\omega}=E \psi_{\omega}$, for spectrally almost all energies and fixed $\omega$. Secondly, the probabilistic estimates required for the inductive step, presented in section 8.3, are different. We present these to contrast and compare them with our hypotheses $[\mathrm{H} 1]\left(\gamma_{0}, \ell_{0}\right)$ and $[\mathrm{W}]$.

The basic finite-volume Hamiltonian assumption used by von Dreifus and Klein $[\mathbf{1 3 2}]$ is the following.

[vDK1]. Let $I \in \mathbb{R}$ be an energy interval, and let $p>d, L_{0}>0$, and take $1<\alpha<2 p / d$. We set $L_{k+1} \equiv L_{k}^{\alpha}$. Then, for any $k=0,1, \ldots$, we have

$$
\mathbb{P}\left\{\text { for any } E \in I \text {, either } \Lambda_{k}(x) \text { or } \Lambda_{k}(y) \text { is } \gamma-\operatorname{good} \text { at energy } E\right\} \geq 1-L_{k}^{-p},
$$

for any $x, y \in \Gamma_{k}$ with $\|x-y\|>L_{k}$.

Note that unlike our hypotheses, control over the resolvent is required at all energies $E \in I$. Given hypothesis [vDK1], the main step replacing the Theorem 5.3 , and the perturbation of singular spectra analysis of chapter 3 , is the use of the generalized eigenfunctions associated with $H_{\omega}$. A generalized eigenfunction at energy $E$ is a nonzero, polynomially bounded solution of the equation $H \psi=E \psi$. An energy $E$ for which there exists a generalized eigenfunction is called a generalized eigenvalue. The main result about these generalized eigenfunctions is the following theorem. A proof of this theorem can be found, for example, in [35].

TheOREM 8.2. Let $H$ be a self-adjoint operator on a separable Hilbert space. With respect to the spectral measure of $H$, almost every energy $E \in \mathbb{R}$ is a generalized eigenvalue.

One defines a set $\Omega_{0}$ of configurations with $\mathbb{P}\left(\Omega_{0}\right)=1$, and for which not too many singular sites occur for the corresponding local Hamiltonians. One then proves that for $\omega \in \Omega_{0}$, the generalized eigenfunctions of $H_{\omega}$ decay, in fact, exponentially fast. Hence, they are eigenfunctions.

Condition (8.2) is not easy to prove directly. As in chapter 5 , it is reduced to a consequence of two fixed length-scale hypotheses. The inductive part of the multiscale analysis then involves showing that these two fixed length-scale hypotheses 
imply condition (8.2). The fixed length-scale hypotheses of von Dreifus and Klein $[\mathbf{1 3 2}]$ are the following.

$[\mathbf{P 1}]$. There exists an $L_{0}>0$, so that for some $E_{0} \in \mathbb{R}$, we have

$$
\mathbb{P}\left\{\Lambda_{L_{0}}(0) \text { is } \gamma-\text { good at energy } E_{0}\right\} \geq 1-L_{0}^{-p},
$$

for some $p>d$.

[P2]. For some $\eta>0$, and for all $E \in\left(E_{0}-\eta, E_{0}+\eta\right)$, and for all $L>L_{0}$, there is a $\beta$ satisfying $0<\beta<1$, and a $q>4 p+6 d$, such that

$$
\mathbb{P}\left\{\operatorname{dist}\left(E, \sigma\left(H_{\Lambda_{L}(0)}\right)<e^{L^{\beta}}\right\} \leq L^{-q} .\right.
$$

These two hypotheses correspond to our hypotheses $[\mathrm{H} 1]\left(\gamma_{0}, \ell_{0}\right)$ and $[\mathrm{W}]$, respectively. It is easy to show by using the first resolvent equation that condition [P1] can be extended to a small interval of energy about $E_{0}$. Condition [P2] is similar to the Wegner estimate $[\mathrm{W}]$. A Wegner estimate at any fixed energy can always be extended to an interval about that energy.

8.3. The Inductive Step. The key to the proof of Lemma 5.4 is an inductive step. The proof of this step goes back to the fundamental work of Fröhlich and Spencer [56], and was successively refined by Spencer [120] and von Dreifus and Klein [132]. Like most of the proofs in the field of random operators, it has two components. First, one makes an assumption about the scarcity of regions which will produce eigenvalues for the local Hamiltonians close to the energy of interest. These regions are called resonant regions. Their presence causes small denominators in the iterated geometric resolvent equation. Second, one computes the probability that the bad, resonant regions occur.

For a given $l$, we will consider a covering of $\mathbb{R}^{d}$ by boxes $\Lambda_{l / 3}(x)$, centered at points $x$ on a scaled lattice $\Gamma_{\ell} \equiv \frac{l}{3} \ell \mathbb{Z}^{d}$. In particular, the box $\Lambda_{l}(0)$ is covered by $3^{d}$ such boxes of side length $\ell / 3$. We define a family of $n$ chained $l$ boxes to be a sequence of boxes $\Lambda_{l}\left(x_{k}\right), k=1, \ldots, n$, such that $x_{k} \in \Gamma_{l} \cap \Lambda_{l}\left(x_{k-1}\right)$ and $x_{k} \neq x_{k-1}$. For later purposes, we note that $d\left(x_{k}, \partial \Lambda_{l}\left(x_{k-1}\right)\right)=\frac{1}{6} l$, and that $\left|x_{n}-x_{1}\right| \leq \frac{\sqrt{2}}{3}(n-1) l$. Whenever convenient, we write $\chi_{l}, R_{l}$, etc. for $\chi_{\Lambda_{l}}, R_{\Lambda_{l}}$, etc. Let us recall that for a fixed $\delta>0$, we define a subbox $\tilde{\Lambda}_{l}$ of $\Lambda_{l}$ by $\tilde{\Lambda}_{l} \equiv\{x \in$ $\left.\Lambda \mid \operatorname{dist}\left(x, \partial \Lambda_{l}\right)>\delta\right\}$.

We single out a family of boxes for which the resolvent of the local Hamiltonian has exponential decay across the box. This condition is roughly equivalent to saying that the eigenvalues of $H_{\Lambda_{l}}$ are far separated from the energy $E$ of interest. if

$$
\sup _{\epsilon>0}\left\|W\left(\chi_{l}\right) R_{\Lambda_{l}}(E+i \epsilon) \chi_{\Lambda_{l / 3}}\right\|<e^{-\gamma l} .
$$

We show that if there are not too many disjoint $\gamma$-bad $l$-boxes $\Lambda_{l}$ in a bigger box $\Lambda_{l^{\prime}}$, then the assumption that $\Lambda_{l}$ is $\gamma$-good implies that $\Lambda_{l^{\prime}}$ is $\gamma^{\prime}$-good without losing too much in the exponent $\gamma$.

LEMMA 8.4. Let $l^{\prime}>>4 l$ be chosen large enough and assume [W]. If $\gamma>>d / l$ and

$$
\mathbb{P}\left\{\Lambda_{l} \text { is a } \gamma \text {-good box }\right\} \geq 1-\eta,
$$


then we have

$$
\mathbb{P}\left\{\Lambda_{l^{\prime}} \text { is a } \gamma^{\prime}-\operatorname{good} \text { box }\right\} \geq 1-\eta^{\prime},
$$

where, for a constant $c_{d}$ depending only on $d$ and any $\xi>2 d$,

$$
\eta^{\prime}=c_{d}\left(\frac{l^{\prime}}{l}\right)^{2 d} \eta^{2}+\frac{1}{2}\left(l^{\prime}\right)^{-\xi},
$$

and

$$
\gamma^{\prime}=\left(\gamma-\frac{c_{1}}{l}\right)\left(1-\frac{4 l}{l^{\prime}}\right)-c_{2} \frac{\log l^{\prime}}{l^{\prime}},
$$

for some constants $c_{1}, c_{2}>0$ depending on $d, \xi$, and $C_{W}$.

Proof. Without restricting generality we consider the cube $\Lambda_{l^{\prime}}$ centered at the origin.

1. We first assume that $\Lambda_{q l^{\prime}}$, for $q \geq 2$, does not contain two non-overlapping $\gamma$-bad boxes $\Lambda_{l}$ centered on the scaled lattice $\Gamma_{l}$. This means that there may be only one cluster $\Lambda_{b}$ of overlapping $\gamma$-bad $l$-cubes in $\Lambda_{q l^{\prime}}$ centered on $\Gamma_{l}$. The diameter of this bad region $\Lambda_{b}$ is at most $\frac{5 \sqrt{2}}{3} l$. We now consider a covering of $\Lambda_{l^{\prime} / 3}(0)$ by boxes $\Lambda_{l / 3}$ with centers in $\Gamma_{l} \cap \Lambda_{l^{\prime} / 3}(0)$. There are $\left(l^{\prime} / l\right)^{d}$ such centers and we call $x$ one of them. We also consider a covering of $\Lambda_{l^{\prime}}(0) \backslash \tilde{\Lambda}_{l^{\prime}}(0)$ by such cubes $\Lambda_{\ell / 3}$ with centers in $\Lambda_{q l^{\prime}}(0) \cap \Gamma_{l}$. There are $\left(d \delta / l^{\prime}\right)\left(3 l^{\prime} / l\right)^{d}$ such centers. Let $y$ be one of them so that $\|x-y\| \geq\left(l^{\prime} / 3-\delta / 2\right)$. We want to estimate

$$
\left\|\chi_{l / 3}(x) R_{q l^{\prime}}(E+i \epsilon) \chi_{l / 3}(y)\right\|,
$$

for the multiplier $q \geq 2$. We need the estimate for $q \geq 2$ in order to compensate for the boundary, as we will explain below.

2. We estimate this norm by constructing suitable families of chained $l$-boxes, which are $\gamma$-good $l$-boxes with a certain probability, starting at $x_{1} \equiv x$ or $y_{1} \equiv y$. By iterating the geometric resolvent equation (GRE) presented in section 5.1 (5.11) along such a chain, we obtain an estimate for the decay of the operator in $(8.10)$ due to (8.1) and an a priori estimate derived from the Wegner estimate. In order to begin this process, we write the GRE as

$$
\chi_{l / 3}(x) R_{q l^{\prime}}-R_{l} \chi_{l / 3}(x)=R_{l} W_{l}\left(x_{1}\right) R_{q l^{\prime}} .
$$

Substituting this into (8.10), and using the fact that $\chi_{l / 3}(x) \chi_{l / 3}(y)=0$, we obtain

$$
\chi_{l / 3}\left(x_{1}\right) R_{q l^{\prime}} \chi_{l / 3}(y)=\chi_{l / 3}\left(x_{1}\right) R_{l} W_{l}\left(x_{1}\right) R_{q l^{\prime}} \chi_{l / 3}(y) .
$$

3. Let $\chi_{l}^{(m)}$ be a covering of $\partial \Lambda_{l}\left(x_{1}\right) \equiv \operatorname{supp} W_{l}\left(x_{1}\right)$ by cubes $\Lambda_{l / 3}$ centered on $\Gamma_{l} \cap$ $\Lambda_{l}\left(x_{1}\right)$. One needs $b_{d} \leq(d \delta / l) 3^{d}<3^{d}$ such cubes. We decompose the perturbation $W_{\ell}\left(x_{1}\right)$ appearing in (8.12) as

$$
W_{l}\left(x_{1}\right)=\sum_{m=1}^{b_{d}} W_{l}\left(x_{1}\right) \chi_{l / 3}^{(m)}
$$

Substituting this into (8.12), one obtains from (8.10)

$$
\begin{aligned}
& \left\|\chi_{l / 3}\left(x_{1}\right) R_{q l^{\prime}} \chi_{l / 3}\left(y_{1}\right)\right\| \\
& \leq b_{d}\left\|\chi_{l / 3}\left(x_{1}\right) R_{l} W_{l}\left(x_{1}\right)\right\|\left\{\max _{m}\left\|\chi_{l / 3}^{(m)} R_{q l^{\prime}} \chi_{l / 3}\left(y_{1}\right)\right\|\right\} .
\end{aligned}
$$


If $x_{1} \notin \Lambda_{b}$ and if $x_{2}$ denotes the center in $\Gamma_{l}$ for which the maximum on the right hand side of (8.14) is reached, one obtains

$$
\left\|\chi_{l / 3}\left(x_{1}\right) R_{q l^{\prime}} \chi_{l / 3}\left(y_{1}\right)\right\| \leq b_{d} e^{-\gamma l}\left\|\chi_{l / 3}\left(x_{2}\right) R_{q l^{\prime}} \chi_{l / 3}\left(y_{1}\right)\right\| .
$$

4. If $x_{2} \notin \Lambda_{b}$ and $\Lambda_{l}\left(x_{2}\right) \cap \Lambda_{l / 3}\left(y_{1}\right)=\emptyset$, one can continue this procedure and construct in this way a family of $n$ chained $\gamma$-good boxes $\Lambda_{l}\left(x_{k}\right), k=1, \ldots, n$. The chain is stopped when $d\left(x_{n}, \Lambda_{b}\right)=l / 6$ or $\left\|x_{n}-y\right\|=l / 3$. In the case that $\left\|x_{n}-y\right\|=l / 3$, the number of elements in the chain satisfies $n \geq 3\|x-y\| / l$ so that, assuming $\gamma>\log b_{d} / l$ :

$$
\left\|\chi_{l / 3}\left(x_{1}\right) R_{q l^{\prime}} \chi_{l / 3}(y)\right\| \leq e^{-3\left(\gamma-\left(\log b_{d}\right) / l\right)\|x-y\|}\left\|\chi_{l / 3}\left(x_{n}\right) R_{q l^{\prime}} \chi_{l / 3}(y)\right\| .
$$

In the first case when $d\left(x_{n}, \Lambda_{b}\right)=l / 6$, one starts a new chain $\left(y_{1}, \ldots, y_{m}\right)$ from $y_{1}=y$. Once again, the chain is stopped when $d\left(y_{m}, \Lambda_{b}\right)=l / 6$ or $\left|x_{n}-y_{m}\right|=l / 3$. In this last case, the number of sites in the chain starting at $y_{1}$ satisfies $m \geq$ $3\left|x_{n}-y_{1}\right| / l$, so that $m+n \geq 3\|x-y\| / l$, and an inequality like (8.16), with $y_{m}$ instead of $y$, still holds. In the first case for which $d\left(y_{m}, \Lambda_{b}\right)=l / 6$, we find that

$$
(m+n-2) \frac{l}{3}+2 l \geq\|x-y\|
$$

and one obtains

$$
\left\|\chi_{l / 3}(x) R_{q l^{\prime}} \chi_{l / 3}(y)\right\| \leq e^{-\left(\gamma-\left(\log b_{d} / l\right)[3\|x-y\|-4 l]\right.}\left\|R_{q l^{\prime}}\right\| .
$$

Note that $\|x-y\| \geq l^{\prime} / 3$. We now turn this estimate into an estimate for

$$
\left\|\chi_{l^{\prime} / 3} R_{q l^{\prime}}(E+i \epsilon) \tilde{\chi}_{l^{\prime}}\right\| .
$$

We use our covering of $\Lambda_{l^{\prime} / 3}$ and of the boundary region $\Lambda_{l^{\prime}} \backslash \tilde{\Lambda}_{l^{\prime}}$. Since each term satisfies an identical estimate, we obtain

$$
\left\|\chi_{l^{\prime} / 3} R_{q l^{\prime}}(E+i \epsilon) \tilde{\chi} l^{\prime}\right\| \leq \frac{\delta d}{l^{\prime}} 3^{d}\left(\frac{l^{\prime}}{l}\right)^{2 d} e^{-\left(\gamma-\left(\log b_{d}\right) / l\right)\left(l^{\prime}-4 l\right)}\left\|R_{q l^{\prime}}(E+i \epsilon)\right\|,
$$

where $\tilde{\chi}_{l^{\prime}}$ is a characteristic function of the boundary region $\Lambda_{l^{\prime}} \backslash \tilde{\Lambda}_{l^{\prime}}$.

5. The main reason for introducing $q \geq 2$ is twofold. First, we must make sure we can cover all of the boundary region $\Lambda_{l^{\prime}} \backslash \tilde{\Lambda}_{l^{\prime}}$ with cubes $\Lambda_{l / 3}$ centered on the lattice $\Gamma_{l}$. Secondly, in the case when the chain starting at $x_{1}$ stops because it meets the bad regions $\Lambda_{b}$, we must be sure that we can iterate the chain starting at $y_{1}$ sufficiently far enough so that the combination $(n+m)$ satisfies an estimate like (8.17). For example, it might occur that a chain starting at $y_{1}$ in the boundary region has to be stopped because it hits the boundary of $\Lambda_{l^{\prime}}$ before $m$ is large enough. To avoid this, we continue to iterate into the expanded region $\Lambda_{q l^{\prime}}$, if necessary. The price that we pay for this is not serious: we obtain a factor $q^{d}$ in the coefficient of the resolvent estimate and in the probability estimate. Since these constants are independent of the length scale, they do not affect the results.

6. From (8.20), one obtains a similar bound for $\left\|\chi_{l^{\prime} / 3} R_{q l^{\prime}}(E+i \epsilon) W_{l^{\prime}}\right\|$, with an extra multiplicative constant which only depends on $\left\|\nabla \chi_{l^{\prime}}\right\|_{\infty}$ and $\left\|\Delta \chi_{l^{\prime}}\right\|_{\infty}$. This 
is easily seen by writing $W_{l^{\prime}}=-2 \nabla \cdot \nabla \chi_{l^{\prime}}-\Delta \chi_{l^{\prime}}$, and using the following inequality:

$$
\begin{aligned}
\left\|\nabla\left(\nabla \chi_{l^{\prime}} u\right)\right\|^{2}= & 2 \operatorname{Re}\left\langle-\Delta u,\left|\nabla \chi_{l^{\prime}}\right|^{2} u\right\rangle+2\left\|\left(\Delta \chi_{l^{\prime}}\right) u\right\|^{2} \\
\leq & 2\left|\operatorname{Re}\left\langle\left(H_{\Lambda_{q l^{\prime}}}-E-i \epsilon\right) u,\left|\nabla \chi_{l^{\prime}}\right|^{2} u\right\rangle\right|+2 \sum_{i=1}^{d}\left\|\left|\nabla \partial_{i} \chi_{l^{\prime}}\right| u\right\|^{2} \\
& +2\left|\operatorname{Re}\left\langle\left(E-V_{0}-V_{\Lambda_{q l^{\prime}}}\right) u,\left|\nabla \chi_{l^{\prime}}\right|^{2} u\right\rangle\right| .
\end{aligned}
$$

This inequality can be proved using the equality

$$
\|\nabla(\psi u)\|^{2}=\operatorname{Re}\left\langle\nabla u, \nabla\left(\psi^{2} u\right)\right\rangle+\||\nabla \psi| u\|^{2},
$$

which can be found in the book by Agmon [1] on page 20, equation (1.16). Taking $u=R_{q l^{\prime}}(E+i \epsilon) \chi_{l^{\prime} / 3} \psi$, for any $\psi \in L^{2}\left(\mathbb{R}^{d}\right)$, and using the fact that $\left|\nabla \chi_{l^{\prime}}\right| \chi_{l^{\prime} / 3}=0$, we finally obtain

$$
\begin{aligned}
\left\|W_{l^{\prime}} R_{q l^{\prime}}(E+i \epsilon) \chi_{l^{\prime} / 3}\right\| \leq & \left(2 \sum_{i, j=1}^{d}\left\|\partial_{i} \partial_{j} \chi_{l^{\prime}}\right\|_{\infty}+2\left\|E-V_{\Lambda_{l^{\prime}}}-V_{0}\right\|_{\infty}^{1 / 2}\left\|\nabla \chi_{l^{\prime}}\right\|_{\infty}^{2}\right. \\
& \left.+2\left\|\nabla \chi_{l^{\prime}}\right\|_{\infty}\left\|\Delta \chi_{l^{\prime}}\right\|_{\infty}\right) \\
& \times\left\|\chi_{l^{\prime}} R_{q l^{\prime}}(E+i \epsilon) \chi_{l^{\prime} / 3}\right\| .
\end{aligned}
$$

Here, we take a function $\tilde{\chi}_{l^{\prime}}$ with a slightly larger support so that $\tilde{\chi}_{l^{\prime}} \chi_{l^{\prime}}=\chi_{l^{\prime}}$.

7. Next, we use the GRE to pass from the result for $R_{q l^{\prime}}$ to one for $R_{l^{\prime}}$. As we have

$$
\chi_{l^{\prime}} R_{q l^{\prime}}-R_{l^{\prime}} \chi_{l^{\prime}}=R_{l^{\prime}} W_{l^{\prime}} R_{q l^{\prime}},
$$

we obtain

$$
W_{l^{\prime}} R_{q l^{\prime}} \chi_{l^{\prime} / 3}=W_{l^{\prime}} R_{l^{\prime}} \chi_{l^{\prime} / 3}+W_{l^{\prime}} R_{l^{\prime}} W_{l^{\prime}} R_{q l^{\prime}} \chi_{l^{\prime} / 3} .
$$

We rewrite this equation to obtain a bound involving some constant $C$, depending only on $\left\|\nabla \chi_{l^{\prime}}\right\|_{\infty},\left\|\Delta \chi_{l^{\prime}}\right\|_{\infty}$, and $E$, similar to the coefficient on the right side of (8.23),

$\left\|W_{l^{\prime}} R_{l^{\prime}}(E+i \epsilon) \chi_{l^{\prime} / 3}\right\| \leq C\left(1+\left\|R_{l}(E+i \epsilon) \chi_{l^{\prime} / 3}\right\|\right)\left\|\tilde{\chi}_{l^{\prime}} R_{q l^{\prime}} \chi_{l^{\prime} / 3}\right\|+\left\|W_{l^{\prime}} R_{q l^{\prime}}(E+i \epsilon) \chi_{l^{\prime} / 3}\right\|$.

8. Finally, we use the Wegner estimate $[\mathrm{W}]$ to bound the norm of the resolvent on the right side of (8.23) and of (8.26) by

$$
\left\|R_{p l^{\prime}}(E+i \epsilon)\right\| \leq 2 C_{W} p^{d}\left(l^{\prime}\right)^{\xi+d} \text {, for } p=1 \text { or } q .
$$

This estimate holds with probability at least $1-\frac{1}{2}\left(l^{\prime}\right)^{-\xi}$, provided $l$, and hence $l^{\prime}$, is large enough. We have obtained the following estimate on the resolvent of the local Hamiltonian $H_{\Lambda_{l^{\prime}}}$ from (8.26)-(8.27),

$$
\left\|W_{l^{\prime}} R_{l^{\prime}}(E+i \epsilon) \chi_{l^{\prime} / 3}\right\| \leq e^{-\gamma^{\prime} l^{\prime}}
$$

with

$$
\gamma^{\prime}=\left(\gamma-\frac{\log b_{d}}{l}\right)\left(1-\frac{4 l}{l^{\prime}}\right)-c \frac{\log l^{\prime}}{l^{\prime}},
$$

with a constant $c$ depending only on $d, C_{W}$, and $\xi$. Recall that $\xi>2 d$, so $\xi$ can be taken to be a simply a multiple of $d$. This holds provided the resolvent estimate (8.27) is satisfied, and there are not two disjoint or more $\gamma$-bad $\Lambda_{l}$ boxes centered in $\Gamma_{l} \cap \Lambda_{q l^{\prime}}$. The probability of this last event is larger than $1-c_{d}\left(l^{\prime} / l\right)^{2 d} \eta^{2}$, where $c_{d}$ is a combinatorial factor depending only on $d$ and $q$. Here, we use the independence of events associated with disjoint regions. Consequently, our localized resolvent 
estimate (8.28) holds with probability $\geq 1-c_{d} \eta^{2}\left(l^{\prime} / l\right)^{2 d}-\frac{1}{2}\left(l^{\prime}\right)^{-\xi}$. This concludes the proof of Lemma 8.4.

8.4. Consequences of the Inductive Step. We prove Lemma 5.4 from the inductive step described in Lemma 8.4.

there

Proof of Lemma 5.4. Assuming $l_{0}$ is large enough, say $l_{0} \geq l_{1}^{*}$, one can define $\gamma_{k}$ inductively according to (8.9) in Lemma 8.4,

$$
\gamma_{k+1}=\gamma_{k}\left(1-\frac{4 l_{k}}{l_{k+1}}\right)-c_{k}
$$

where

We find

$$
c_{k}=\frac{c_{1}}{l_{k}}\left(1-\frac{4 l_{k}}{l_{k+1}}\right)+c_{2} \frac{\log l_{k+1}}{l_{k+1}} .
$$

$$
\gamma_{k+1}=\gamma_{0} \prod_{j=0}^{k}\left(1-N_{j}\right)-\sum_{l=0}^{k} \prod_{j=0}^{(l-1)}\left(1-N_{j}\right) c_{k-l}
$$

with $N_{j}=4 l_{j} / l_{j+1}$. It is easy to check that $\prod_{j=0}^{k}\left(1-N_{j}\right)$ is uniformly bounded below by a strictly positive number $\kappa$ uniformly in $l_{0}$ if $l_{0} \geq 2$. The subtracted term in (8.31) is certainly $\leq \kappa \gamma_{0} / 2$ if $l_{0}$ is large enough, say $l_{0} \geq l_{2}^{*}$, so that $\gamma_{k} \geq \kappa \gamma_{0} / 2$ for all $k$. According to Lemma 8.4, suppose $\Lambda_{k}$ is $\gamma_{k}$-good with probability $\geq 1-\eta_{k}$, where $\eta_{k}=l_{k}^{-\xi}$. We compute an upper bound on $\eta_{k+1}$ using the definition given in (8.8),

$$
\begin{aligned}
\eta_{k+1} & =c_{d} l_{k}^{(d-2 \xi)}+\frac{1}{2} l_{k+1}^{-\xi}=c_{d}\left(l_{k+1}\right)^{2(d-2 \xi) / 3}+\frac{1}{2} l_{k+1}^{-\xi} \\
& \leq\left(\frac{1}{2}+c_{d} l_{k+1}^{(2 d-\xi / 3)}\right) l_{k+1}^{-\xi} .
\end{aligned}
$$

This is less than $l_{k+1}^{-\xi}$ since $\xi>2 d$ for $l_{0}>l_{3}^{*}=\left(2 c_{d}\right)^{(\xi-2 d) / 3}$. In these last estimates, we have used the identity $l_{k+1}=l_{k}^{3 / 2}$. We obtain the result with $l^{*}=$ $\max \left\{l_{i}^{*}, i=1,2,3\right\}$.

We now give a refinement of these lemmas needed in the proof of Theorem 5.4.

LEMMA 8.5. Let $\gamma_{0}>0$ and assume $[W]$. Let $l^{*}$ be the minimum length scale of Lemma 5.4 and suppose $[\mathrm{H} 1]\left(\gamma_{0}, l_{0}\right)$ holds for $l_{0}>l^{*}$. Then, there exists a constant $C_{p}=C_{p}\left(\gamma_{0}, C_{W}, d\right)$, such that for any cube $\Lambda \subset \mathbb{R}^{d}$ sufficiently large so that $\Lambda_{q l_{k}} \subset \Lambda$, for some large $k$ and $q \geq 2$, and $x, y \in \Lambda$ such that

$$
\operatorname{dist}(x \text { or } y, \partial \Lambda)>\|x-y\|>q l_{k}
$$

and

$$
q l_{k+1}>\|x-y\|>q l_{k},
$$

one has

$$
\mathbb{P}\left\{\sup _{\epsilon>0}\left\|\chi_{x} R_{\Lambda}(E+i \epsilon) \chi_{y}\right\| \leq e^{-(1 / 2 \sqrt{d}) \gamma_{0}\|x-y\|}\right\} \geq 1-C_{p}|\Lambda| q^{2 d}\|x-y\|^{-\xi},
$$


where $\chi_{x}, \chi_{y}$ are characteristic functions of unit cubes centered at $x$ or $y$ and we write $\gamma_{0}$ for $\kappa \gamma_{0} / 2$ of Lemma 8.4 for simplicity.

Proof. Let us suppose dist $(x, \partial \Lambda) \geq \operatorname{dist}(y, \partial \Lambda)$; in the other case we begin the construction at $y$. We assume without restricting generality that $x, y \in \Gamma_{l_{k}}$. As in Lemma 8.4 we can find a least $n$ chained boxes of side $l_{k}$ centered on points in $\Gamma_{l_{k}}$ starting at $x$ before reaching $\partial \Lambda$ or $y$ where $n \geq 3\|x-y\| / l_{k}$. By Lemma 8.4, the probability that each box $\Lambda_{l_{k}}$ is $\gamma_{0}$-good is $\geq 1-l_{k}^{-\xi}$. As in the proof of Lemma 8.4 ,

$$
\sup _{\epsilon>0}\left\|\chi_{l_{k} / 3}(x) R_{\Lambda}(E+i \epsilon) \chi_{l_{k} / 3}(y)\right\| \leq e^{-(3 / 4 \sqrt{d}) \gamma_{0}\|x-y\|}\left\|R_{\Lambda}(E+i \epsilon)\right\|,
$$

with probability $\geq 1-\eta l_{k}^{-\xi} \geq 1-l_{k}^{-\xi}|\Lambda| c_{d}$, where $c_{d}$ depends only on $d$. Here we have assumed that $k$ is large enough so that

$$
\gamma_{0}-\frac{\log b_{d}}{l_{k}}>\frac{3 \gamma_{0}}{4}
$$

We use Wegner's lemma $[\mathrm{W}]$ to estimate $\left\|R_{\Lambda}\right\|$ and obtain

$$
\mathbb{P}\left\{\left\|R_{\Lambda}(E+i \epsilon)\right\| \leq e^{(1 / 4 \sqrt{d}) \gamma_{0} l_{k}}\right\} \geq 1-C_{W}|\Lambda| e^{-(1 / 4 \sqrt{d}) \gamma_{0} l_{k}} .
$$

Estimates (8.36)-(8.37) yield

$$
\sup _{\epsilon>0}\left\|\chi_{l_{k} / 3}(x) R(\Lambda)(E+i \epsilon) \chi_{l_{k} / 3}(y)\right\| \leq e^{-(1 / 2 \sqrt{d}) \gamma_{0}\|x-y\|},
$$

with probability $\geq 1-|\Lambda|\left\{C_{W} e^{-(1 / 4 \sqrt{d}) \gamma_{0} l_{k}}+l_{k}^{-\xi}\right\} \geq 1-c_{p}|\Lambda| q^{2 d}\|x-y\|^{-2 d}$, where we have used the identity $l_{k}=\left(l_{k+1}\right)^{2 / 3}$.

\section{References}

[1] S. Agmon, Lectures on exponential decay of solutions of second-order elliptic equations, Mathematical Notes 29, Princeton University Press, Princeton, NJ, 1982.

[2] Aizenman:1994 M. Aizenman: Localization at weak disorder: some elementary bounds, Rev. Math. Phys. 6, No. 5a, 1163-1182 (1994).

[3] M. Aizenman, A. Elgart, S. Naboko, J. H. Schenker, G. Stolz: Moment analysis for localization in random Schrödinger operators, Invent. Math. 163, 343-413 (2006).

[4] M. Aizenman, S. Molchanov: Localization at large disorder and at extreme energies: an elementary derivation, Commun. Math. Phys. 157, 245-278 (1993).

[5] M. Aizenman, R. Sims, S. Warzel: Stability of the absolutely continuous spectrum of random Schrödinger operators on tree graphs, Probab. Theory Related Fields 136, 363394 (2006).

[6] P. W. Anderson, Absence of diffusion in certain random lattices, Physical Review 109, 1492-1505 (1958).

[7] N. Aronszajn: On a problem of Weyl, Amer. J. Math. 79, 596-620 (1957).

[8] J.-M. Barbaroux, J. M. Combes, and P. D. Hislop: Localization near band edges for random Schrödinger operators, Helv. Phys. Acta 70, 16-43 (1997).

[9] J.-M. Barbaroux, J. M. Combes, and P. D. Hislop: Landau Hamiltonians with unbounded random potentials, Letts. Math. Phys. 40, 355-369 (1997).

[10] J.-M. Barbaroux, J. M. Combes, and R. Montcho, Remarks on the relation between quantum dynamics and fractal spectra, J. Math. Anal. Appl. 213, 698-722 (1997).

[11] J.- M. Barbaroux, thèse, Université de Toulon et du Var 1997.

[12] J.-M. Barbaroux, F. Germinet, S. Tcheremchantsev: Fractal dimensions and the phenomenon of intermittency in quantum dynamics, Duke Math. J. 110, 161-193 (2001).

[13] D. Belitz and T. R. Kirkpatrick: The Anderson-Mott Transition, Rev. of Modern Phys. 66, 261 (1994).

[14] J. Bellissard: Ordinary quantum Hall effect and non-commutative cohomology, in Localization in Disordered Systems, W. Weller and P. Zieche, eds., Leipzig: Teubner 1988. 
[15] J. Bellissard, A. van Elst, and H. Schulz-Baldes, The noncommutative geometry of the quantum Hall effect, J. Math. Phys. 35, 5373-5451 (1994).

[16] M. S. Birman, M. Z. Solomjak: Spectral Theory of Self-Adjoint Operators in Hilbert Space, Dordrecht: D. Reidel Publishing Co. 1987.

[17] M. S. Birman, D. R. Yafaev: The spectral shift function: The work of M. G. Krein and its further development, St. Petersburg Mathematics Journal 4, 833-870 (1992).

[18] Ph. Briet, J. M. Combes, P. Duclos: Spectral stability under tunneling, Commun. Math. Phys., 126, 133-186 (1989).

[19] J. Bourgain, C. Kenig: On localization in the continuous Anderson-Bernoulli model in higher dimension, Invent. Math. 161, 389-426 (2005).

[20] A. Boutet de Monvel, S. Naboko, P. Stollmann, G. Stolz: Localization near fluctuation boundaries via fractional moments and applications, J. d'Anal. (2007).

[21] R. Carmona, J. Lacroix: Spectral theory of random Schrödinger operators, Birkhaüser, Boston, 1990.

[22] J. L. Chayes and L. Chayes, Percolation and Random Media, in Critical Phenomena, Random Systems, and Gauge Theories, Proc. les Houches Summer School, Session XLIII, $1000-1142(1984)$.

[23] J. M. Combes: Connections between quantum dynamics and spectral properties of timeevolution operators, in Differential equations with applications to mathematical physics, 59-68, Math. Sci. Engrg., 192, Academic Press, Boston, MA, 1993

[24] J. M. Combes, P. D. Hislop: Some Transport and Spectral Properties of Disordered Media, in Schrödinger Operators: The Quantum Many-body Problem, E. Balslev (ed.), 16-47, Berlin: Springer Verlag (1992).

[25] J. M. Combes, P. D. Hislop: Localization for some continuous random Hamiltonians in d-dimensions, J. Funct. Anal. , 124, 149-180 (1994).

[26] J. M. Combes, P. D. Hislop: Landau Hamiltonians with random potentials: Localization and density of states, Commun. Math. Phys., 177, 603-629 (1996).

[27] J. M. Combes, P. D. Hislop, F. Klopp: Hölder continuity of the integrated density of states for some random operators at all energies, Int. Math. Res. Not. 2003, no. 4, 179-209.

[28] J. M. Combes, P. D. Hislop, F. Klopp: An optimal Wegner estimate and its application to the global continuity of the integrated density of states for random Schrödinger operators, to appear in the Duke Mathematical Journal, 2007.

[29] J. M. Combes, P. D. Hislop, F. Klopp, G. Raikov: Global continuity of the integrated density of states for random Landau Hamiltonians, Comm. Partial Differential Equations 29, 1187-1213 (2004).

[30] J. M. Combes, P. D. Hislop, E. Mourre: Spectral averaging, perturbation of singular spectra, and localization, Trans. Am. Math. Soc., 348, 4883-4894 (1996).

[31] J. M. Combes, P. D. Hislop, E. Mourre: Correlated Wegner inequalities for random Schrödinger operators, in Advances in Differential Equations and Mathematical Physics, Proceedings Int. Conf. Diff. Eqns. and Math. Phys., 1997, E. Carlen, E. M. Harrell, and M. Loss, eds., Contemporary Mathematics Vol. 217, American Mathematical Society, Providence, RI, 1998.

[32] J. M. Combes, P. D. Hislop, S. Nakamura: The $L^{p}$-theory of the spectral shift function, the Wegner estimate, and the integrated density of states for some random operators, Comm. Math. Phys. 218, 113-130 (2001).

[33] J. M. Combes, P. D. Hislop, and A. Tip: Band edge localization and the density of states for electromagnetic and acoustic waves in random media, Ann. Inst. H. Poincaré 70, 381-428 (1999).

[34] J. M. Combes, L. Thomas: Asymptotic behaviour of eigenfunctions for multiparticle Schrödinger operators, Commun. Math. Phys. 34, 251-270 (1973).

[35] H. L. Cycon, R. G. Froese, W. Kirsch, B. Simon: Schrödinger operators. Berlin : SpringerVerlag, 1987.

[36] E. B. Davies: Spectral theory and differential operators. Cambridge: Cambridge University Press, 1995.

[37] P.A. Deift, R. Hempel: On the existence of eigenvalues of the Schrödinger operator $H+\lambda W$ in a gap of $\sigma(H)$, Commun. Math. Phys. 103, 461-490 (1986).

[38] F. Delyon, Y. Levy, B. Souillard: Anderson localization for multidimensional systems at large disorder or large energy, Comm. Math. Phys. 100, 463-470 (1985). 
[39] W. Donoghue: On the perturbation of spectra, Comm. Pure Appl. Math. 18, 559-579 (1965).

[40] T. C. Dorlas, N. Macris, J. V. Pulé: Localization in a Single-Band Approximation to Random Schrödinger Operators with Magnetic Field, Helv. Phys. Acta. 68 , 329-364 (1995).

[41] T. C. Dorlas, N. Macris, J. V. Pulé: Localization in single Landau Bands, J. Math. Phys. 37, 1574-1595 (1996).

[42] H. J. S. Dorren and A. Tip, Maxwell's equations for non-smooth media; fractal-shaped and pointlike objects, J. Math. Phys. 32 , 3060 (1991 ).

[43] W. Faris: Localization for a random discrete wave equation, in Random Media, IMA Volume 7, G. Papanicolaou, ed., Springer-Verlag, New York, 1987.

[44] W. Faris: A localization principle for multiplicative perturbations, J. Funct. Anal. 67 (1986), 105-114.

[45] A. Figotin, A. Klein: Localization phenomenon in gaps of the spectrum of random lattice operators, J. Stat. Phys. 75, 997-1021 (1994).

[46] A. Figotin and A. Klein: Localization of Electromagnetic and Acoustic Waves in Random Media: Lattice Models, J. Stat. Phys. 76, 985-1003 (1994).

[47] A. Figotin and A. Klein: Localization of Classical Waves I: Acoustic Waves, Commun. Math. Phys. 180, 439-482 (1996).

[48] A. Figotin, and A. Klein: Localization of Classical Waves II: Electromagnetic Waves, Commun. Math. Phys. 184, 411-441 (1997).

[49] A. Figotin, and A. Klein: Midgap defect modes in dielectric and acoustic media, SIAM J. Appl. Math. 58, 1748-1773 (1998).

[50] A. Figotin and P. Kuchment: Band-Gap structure of spectra of periodic dielectric and acoustic media. I. scalar Model, SIAM J. Appl. Math. 56, No. 1, 68-88 (1996).

[51] A. Figotin and P. Kuchment: Band-Gap structure of spectra of periodic dielectric and acoustic media. II. 2D Photonic Crystals, SIAM J. Appl. Math. 56, No. 6, 1561-1620 (1996).

[52] W. Fischer, H. Leschke, P. Müller: Towards localization by Gaussian random potentials in multidimensional continuous space, Letts. Math. Phys. 38, 343-348 (1996).

[53] W. Fischer, T. Hupfer, H. Leschke, P. Müller: Existence of the density of states for multidimensional continuum Schrödinger operators with Gaussian random potentials, Commun. Math. Phys. 190, 133-141 (1997).

[54] W. Fischer, H. Leschke, P. Müller: Spectral localization by Gaussian random potentials in multi-dimensional continuous space, J. Statist. Phys. 101, 935-985 (2000).

[55] J. Fröhlich, F. Martinelli, E. Scoppola, T. Spencer: A constructive proof of localization in Anderson tight binding model, Commun. Math. Phys. 101, 21-46 (1985).

[56] J. Fröhlich and T. Spencer: Absence of diffusion in the Anderson tight binding model for large disorder or low energy, Commun. Math. Phys. 88, 151-184 (1983).

[57] R. Froese, D. Hasler, W. Spitzer: Absolutely continuous spectrum for the Anderson model on a tree: a geometric proof of Klein's theorem, Commun. Math. Phys. 269, 239-257 (2007).

[58] F. Germinet, S. DeBièvre: Dynamical localization for discrete and continuous random Schrödinger operators, Commun. Math. Phys. 194, 323-341 (1998).

[59] F. Germinet, P. D. Hislop, A. Klein: On localization for the Schrödinger operator with a Poisson random potential, C. R. Math. Acad. Sci. Paris 341, 525-528 (2005).

[60] F. Germinet, P. D. Hislop, A. Klein: Localization for Schrödinger operators with Poisson random potential, to appear in J. European Math. Soc. (2007).

[61] F. Germinet, P. D. Hislop, A. Klein: Localization at low energies for attractive Poisson random Schrödinger operators, to appear in CRM Lect. Proc. (2007).

[62] F. Germinet, A. Klein: Bootstrap multiscale analysis and localization in random media, Commun. Math. Phys. 222, 415-448 (2001).

[63] F. Germinet, A. Klein: Explicit finite volume criteria for localization in continuous random media and applications, Geom. Funct. Anal. 13, 1201-1238 (2003).

[64] F. Germinet, A. Klein: Operator kernel estimates for functions of generalized Schrödinger operators, Proc. Amer. Math. Soc. 131, 911-920 (2003).

[65] F. Germinet, A. Klein: New characterizations of the region of complete localization for random Schrödinger operators, J. Stat. Phys. 122, 73-94 (2006). 
[66] F. Germinet, A. Klein, J. Schenker: Dynamical delocalization in random Landau Hamiltonians, to appear in Ann. Math..

[67] F. Gesztesy: Some applications of commutation methods, in Schrödinger operators: Proc. of the Nordic Summer School in Mathematics held at Sandbjerg Slot, Sonderborg, Denmark, 1988, Lecture Notes in Physics No. 345, H. Holden and A. Jensen, eds., Berlin: SpringerVerlag 1989.

[68] F. Ghribi, P. D. Hislop, F. Klopp: Localization for Schrödinger operators with random vector potentials, preprint.

[69] G. Grimmett: Percolation. Springer-Verlag, Berlin, 1980.

[70] B. I. Halperin, Quantized Hall conductance, current-carrying edge states, and the existence of extended states in a two-dimensional disordered potential, Phys. Rev.B 25, 2185 (1982).

[71] P. D. Hislop, F. Klopp: The integrated density of states for some random operators with nonsign definite potentials, J. Funct. Anal. 195, 12-47 (2002).

[72] F. Klopp, S. Nakamura, F. Nakano, Y. Nomura: Anderson localization for 2D discrete Schrödinger operators with random magnetic fields, Ann. Henri Poincaré 4, 795-811 (2003).

[73] P. D. Hislop, F. Klopp, J. Schenker: Continuity with respect to disorder of the integrated density of states, Illinois J. Math. 49, 893-904 (2005).

[74] P. D. Hislop, and S. Nakamura: Stark Hamiltonians with Unbounded Random Potentials, Reviews in Math. Phys. 2, 479-494 (1991).

[75] P. D. Hislop, I. M. Sigal, Introduction to Spectral Theory, with Applications to Schrödinger Operators, Applied Mathematical Sciences series volume 113, Springer, New York, 1996.

[76] J. Howland: Perturbation theory of dense point spectra, J. Funct. Anal. 74, 52-80 (1987).

[77] J. Howland: Quantum stability, in Schrödinger Operators, E. Balslev, ed. , Lecture Notes in Physics No. 403, Springer-Verlag, 1992, pp. 100-122.

[78] D. Hundertmark, R. Killip, S. Nakamura, P. Stollmann, I. Veselić: Bounds on the spectral shift function and the density of states, Comm. Math. Phys. 262, 489-503 (2006).

[79] D. Hundertmark, B. Simon: An optimal $L^{p}$-bound on the Krein spectral shift function, $J$. Anal. Math. 87, 199-208 (2002).

[80] S. John: The localization of light and other classical waves in disordered media, Comments Cond. Mat. Phys. 14 (1988), 193-230.

[81] T. Kato: Perturbation Theory for Linear Operators, Second Edition, Grundlehren der mathematischen Wissenschaften 132, Berlin: Springer-Verlag, 1980.

[82] W. Kirsch: Random Schrödinger Operators: a course, in Schrödinger Operators, Sønderborg DK 1988, ed. H. Holden and A. Jensen. Lecture Notes in Physics Vol. 345, Berlin: Springer 1989.

[83] W. Kirsch: Wegner estimates and localization for alloy-type potentials, Math. Zeit. 221, 507-512 (1996).

[84] W. Kirsch, Krishna M. J. Obermeit: Anderson model with decaying randomness: mobility edge, Math. Z. 235, 421-433 (2000).

[85] W. Kirsch and F. Martinelli, On the spectrum of Schrödinger operators with a random potential, Commun. Math. Phys. 85, 329-350 (1982).

[86] W. Kirsch, P. Stollmann, and G. Stolz: Localization for random perturbations of periodic Schrödinger operators, Random Oper. Stochastic Equations 6, 241-268 (1998).

[87] W. Kirsch, P. Stollmann, and G. Stolz: Anderson localization for random Schrödinger operators with long range interactions, Comm. Math. Phys. 195, 495-507 (1998).

[88] A. Klein: Extended states for the Anderson model on the Bethe lattice, Adv. Math. 133, 163-184 (1998).

[89] A. Klein, S. Molchanov: Simplicity of eigenvalues in the Anderson model, J. Stat. Phys. 122, 95-99 (2006).

[90] F. Klopp: Localization for some continuous random Schrödinger operators, Commun. Math. Phys. 167, 553-569 (1995).

[91] F. Klopp: Localization for semi-classical continuous random Schrödinger operators II: the random displacement model, Helv. Phys. Act. 66, 810-841 (1993).

[92] F. Klopp: Internal Lifshits tails for random perturbations of periodic Schrödinger operators, Duke Math. J. 98, 335-396 (1999). 
[93] S. Kotani: Lyapunov indices determine absolutely continuous spectra of stationary random one-dimensional Schrödinger operators, Stochastic Analysis, K. Ito, ed., North Holland, Amsterdam, 1984, 225-248.

[94] S. Kotani, B. Simon: Localization in general one dimensional systems. II, Commun. Math. Phys. 112, 103-120 (1987).

[95] P. Kuchment, Floquet Theory for Partial Differential Equations, Basel: Birkhäuser Verlag 1993.

[96] H. Kunz: The quantum Hall effect for electrons in a random potential, Commun. Math. Phys. 112, 121 - 145 (1987).

[97] H. Kunz, B. Souillard, Sur le spectre des opérateurs aux differences finis aléatoires, Commun. math. Phys. 78, 201-246 (1980).

[98] Y. Last: Quantum dynamics and decompositions of singular continuous spectra, J. Funct. Anal. 142, 406-445 (1996).

[99] H. Leinfelder, C. G. Simader: Schrödinger operators with singular magnetic vector potentials Math. Z. 176, 1-19 (1981).

[100] F. H. Lin: A uniqueness equation for parabolic equations, Comm. Pure Appl. Math. 43, 127-136 (1990).

[101] F. Martinelli, H. Holden: On absence of diffusion near the bottom of the spectrum for a random Schrödinger operator on $L^{2}\left(R^{\nu}\right)$, Commun. Math. Phys. 93, 197-217 (1984).

[102] F. Martinelli, E. Scoppola: Remark on the absence of absolutely continuous spectrum for $d$-dimensional Schrödinger operators with random potential for large disorder or low energy, Commun. Math. Phys. 97, 465-471 (1985).

[103] I. McGillivray, P. Stollmann, G. Stolz: Absence of absolutely continuous spectra for multidimensional Schrödinger operators with high barriers, Bull. London Math. Soc. 27, 162168 (1995).

[104] R. Montcho, thèse, Université de Toulon et du Var, 1997.

[105] E. Mourre: Absence of singular continuous spectrum for certain self-adjoint operators, Commun. Math. Phys. 78, 391-408 (1983).

[106] L. Pastur: Spectra of random self-adjoint operators, Usp. Math. Nauk. 28, 3 (1973).

[107] L. Pastur, A. Figotin: Spectra of random and almost-periodic operators. Berlin: SpringerVerlag, 1992.

[108] R. E. Prange and S. M. Girvin (eds.): The Quantum Hall Effect, Graduate Texts in Contemporary Physics, New York: Springer-Verlag (1987).

[109] R. Joynt and R. E. Prange: Conditions for the quantum Hall effect, Phys. Rev. B 29, $3303-3317$ (1984).

[110] M. Reed, B. Simon: Methods of Modern Mathematical Physics, I: Functional Analysis, revised and enlarged edition. New York: Academic Press, 1980.

[111] M. Reed, B. Simon: Methods of Modern Mathematical Physics, II: Fourier Analysis, SelfAdjointness. New York: Academic Press, 1975.

[112] M. Reed, B. Simon: Methods of Modern Mathematical Physics, III: Scattering Theory. New York: Academic Press, 1979.

[113] M. Reed, B. Simon: Methods of Modern Mathematical Physics, IV: Analysis of Operators. New York: Academic Press 1981.

[114] B. Simon: Trace ideals and their applications, second edition, Mathematical Surveys and Monographs 120, American Mathematical Society, 2005.

[115] B. Simon: Spectral Analysis of Rank One Perturbations and Applications, Vancouver lectures, 1993, in Mathematical Quantum Theory, II. Schrödinger operators, CRM Proc. Lecture Notes 8, 109-149. Providence: AMS, 1995; reprinted in the second edition of Trace Ideals.

[116] B. Simon: Cyclic vectors in the Anderson model. Special issue dedicated to Elliott H. Lieb, Rev. Math. Phys. 6, 1183-1185 (1994).

[117] B. Simon: $L^{p}$-norms of the Borel transform and the decomposition of measures, Proc. Amer. Math. Soc. 123, 3749-3755 (1995).

[118] B. Simon: Spectral averaging and the Krein spectral shift, Proc. Amer. Math. Soc. 126, 1409-1413 (1998).

[119] B. Simon and T. Wolff: Singular continuous spectrum under rank one perturbation and localization for random Hamiltonians, Comm. Pure Appl. Math. 39, 75-90 (1986). 
[120] T. Spencer: Localization for random and quasi-periodic potentials: J. Stat. Phys. 51, 1009-1019 (1988).

[121] P. Stollmann: Localization for random perturbations of anisotropic periodic media, Israel J. Math. 107, 125-139 (1997).

[122] P. Stollmann: Wegner estimates and localization for continuum Anderson models with some singular distributions, Arch. Math. (Basel) 75 307-311 (2000).

[123] P. Stollmann: Caught by disorder. Bound states in random media, Progress in Mathematical Physics, 20, Birkhüser Boston, Inc., Boston, MA, 2001.

[124] G. Stolz: Localization for Schrödinger operators with effective barriers, J. Funct. Anal. 146, 416-429 (1997)

[125] G. Stolz: Localization for random Schrödinger operators with Poisson potential, Ann. Inst. Henri Poincaré 63, 297-314 (1997).

[126] D. J. Thouless, Localization and the two-dimensional Hall effect, J. Phys. C 14, 3475 3480 (1981).

[127] A. Tip, Absolute continuity of the integrated density of states of the quantum Lorentz gas for a class of repulsive potentials, J. Phys. A: Math. Gen. 27 (1994) 1057-1069.

[128] D. V. van Coevorden, R. Sprik, A. Tip, and A. Lagendijk: Photonic Bandstructure of Atomic Lattices, Phys. Rev. Letters 77, 2412 (1996).

[129] I. Veselíc: Existence of the density of states for some alloy type models with single site potentials that change sign, in Applied mathematics and scientific computing (Dubrovnik, 2001), 301-311, Kluwer/Plenum, New York, 2003.

[130] I. Veselíc: Integrated density of states and Wegner estimates for random Schrdinger operators, in Spectral theory of Schrödinger operators, Contemp. Math. 340, 97-183, Amer. Math. Soc., Providence, RI, 2004.

[131] H. von Dreifus and A. Klein: A new proof of localization in the Anderson tight binding model, Commun. Math. Phys. 124, (1989), 285-299.

[132] H. von Dreifus and A. Klein: Localization for random Schrödinger operators with correlated potentials, Commun. Math. Phys. 140, 133-147 (1991).

[133] W. M. Wang: Microlocalization, Percolation, and Anderson localization for the Magnetic Schrödinger Operator with a Random Potential, J. Funct. Anal. 146, 1-26 (1997).

[134] W. M. Wang: Asymptotic expansion for the density of states of the magnetic Schrödinger operator with a random potential, Comm. Math. Phys. 172, 401-425 (1995).

[135] W. M. Wang: Supersymmetry and density of states of the magnetic Schrödinger operator with a random potential revisited, Comm. Partial Differential Equations 25, 601-679 (2000).

[136] F. Wegner: Bounds on the density of states in disordered systems, Z. Phys. B22, (1981), $9-15$.

[137] D. R. Yafaev: Mathematical Scattering Theory: General Theory, Translations of Mathematical Monographs 105, Providence, RI: American Mathematical Society, 1992.

Department of Mathematics, University of Kentucky, Lexington, KY 40506-0027 USA

E-mail address: hislop@ms.uky.edu 